\title{
15. PALYNOLOGY OF CORES FROM DEEP SEA DRILLING SITES 327, 328, AND 330, SOUTH ATLANTIC OCEAN
}

\author{
Wayne K. Harris, Geological Survey of South Australia, \\ P.O. Box 151, Eastwood, South Australia
}

\begin{abstract}
DSDP Sites 327, 328, and 330 in the South Atlantic on the Falkland Plateau yielded suites of well-preserved palynomorphs (spores, pollen, dinoflagellates, acritarchs, and tasmanitids) from Late Jurassic to early Tertiary sediments. The oldest sediments recorded are from Site 330 and are of Oxfordian age. These sediments are separated from those of the late Neocomian to Aptian by an appreciable hiatus. At both Sites 327 and 330 the Late Jurassic and Early Cretaceous sediments are marginal marine and were deposited under reducing euxinic conditions. Palynomorph assemblages are dominated by terrestrial components. Late Cretaceous and early Tertiary sediments at Sites 327 and 328, on the other hand, reflect deep water environments, and the assemblages are dominated by dinoflagellate cysts.

A comparison of Early Cretaceous assemblages from Site 249, Mozambique Ridge (Indian Ocean) supports continental reconstructions which would place this site near those on the Falkland Plateau.

There is a very strong southern hemisphere component in all assemblages, but the marine elements in particular show marked similarity with those of Western Australia and the Indian Ocean, implying a southern Atlantic circulation linked with that of the Indian Ocean.
\end{abstract}

\section{INTRODUCTION}

Palynomorphs comprising terrestrially derived spores and pollen and a marine component of dinoflagellate cysts, acritarchs, and Prasinophyceae (unicellular algae, tasmanitids) were recovered from DSDP Sites 327, 328, and 330 (Figure 1). The sediments range in age from Late Jurassic through early Tertiary and represent a wide range of environments from nearshore deltaic clastics through to pelagic oozes.

The sample data for these sites and Site 249 (DSDP Leg 25) are listed in Table 1.

A large number of the species observed in these samples are illustrated in Plates 1-24. Systematic studies are in progress and will be presented elsewhere.

Because of the large component of undescribed species, detailed distribution charts are not presented here, but will be included in the systematic papers.

The preservation of palynomorphs is generally very good but there are sequences (e.g., Hole 327A, Cores 13-21) where these fossils are absent.

\section{SITE 327}

Samples from Hole $327 \mathrm{~A}$ drilled at this site fall into two groups separated by a large interval of barren samples. Cores 5-12 are of Late Cretaceous and early Tertiary age and Cores 22-27 are Neocomian to Aptian.

\section{Observations}

Early Tertiary-Cores 5-9

Palynomorphs in this interval are rare and many samples are barren. Core 5 carries abundant tracheid material and a small assemblage with Cleistosphaeridium sp., Hexagonifera sp., and aff. Samlandia. None of these are distinctive enough to indicate an age. No terrestrial palynomorphs were observed.

Cores 8 and 9 carry reasonably diverse assemblages and dinoflagellates amount to about $50 \%$ in Core 8 and $90 \%$ in Core 9. Species include:

1) Spores and pollen

Clavatipollenites $\mathrm{cf}$. $C$. hughesi Couper

Gleicheniidites circinidites (Cookson)

Laevigatosporites ovatus Wilson and Webster

Nothofagidites sp. aff. $N$. incrassatus (Cookson)

Phyllocladidites mawsonii Cookson ex Couper

Podocarpidites ellipticus Cookson

Proteacidites $\mathrm{sp}$.

Stereisporites antiquasporites (Wilson and Webster)

Tricolpites cf. T. prolata Cookson

2) Microplankton

Deflandrea sp. A (also present at DSDP Site 214)

$D$. aff. D. gambangensis Cookson and Eisenack

Eisenackia crassitabulata Cookson and Eisenack

"Michrystridium" cf. M. ambiguum Deflandre 


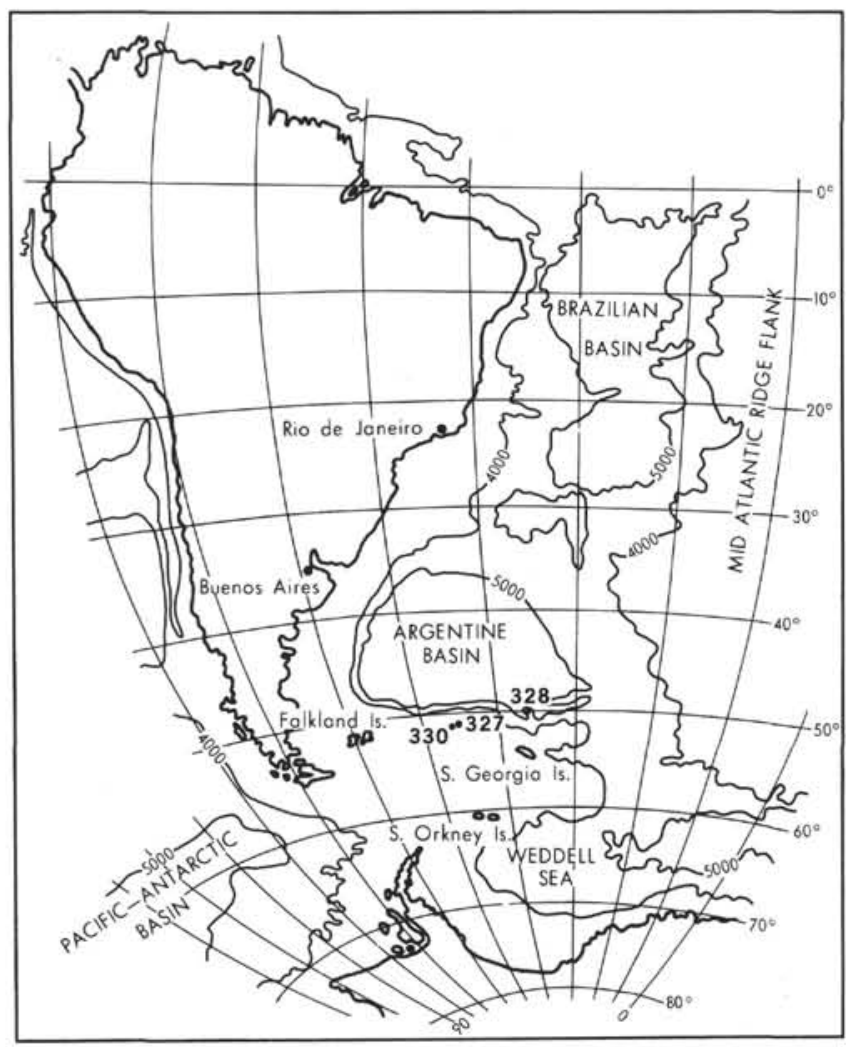

Figure 1. Location of DSDP Sites 327, 328, and 330.

Operculodinium $\mathrm{sp.}$

Palaeoperidinium sp. (see Plate 6, Figure 8)

Spiniferites cingulatus $(\mathrm{O}$. Wetzel)

$S$. ramosus (Ehrenberg)

Wetzeliella homomorpha Deflandre and Cookson Microforaminifera are abundant and Palaeoperidinium sp. is dominant in Core 9 .

\section{Late Cretaceous - Cores 10-12}

There is a very pronounced change between the assemblages of these cores with those above. Spores and pollen are virtually absent.

The assemblage includes:

1) Spores and pollen

Proteacidites sp.

Tricolporites sp.

2) Microplankton

Cannosphaeropsis tutulosa Cookson and Eisenack

(Core 12 only)

Cassiculosphaera sp.

Chlamydophorella $\mathrm{sp}$.

Chytroeisphaeridia sp.

Deflandrea cf. belfastensis Cookson and Eisenack (Abundant in Core 11)

D. nucula Cookson and Eisenack

Eisenackia crassitabulata

Gillinia hymenophora Cookson and Eisenack

(Core 12 only)

Membranosphaera sp. A

Odontochitina sp. A (see Plate 19, Figure 1)

Operculodinium sp.

Spiniferites ramosus
TABLE 1

Data on Samples Studied

Sample

(Interval in $\mathrm{cm}$ ) Sample No.

Hole $327 \mathrm{~A}$ (lat $50^{\circ} 52.28^{\prime} \mathrm{S}$, long $46^{\circ} 47.02^{\prime} \mathrm{W}$

$5, \mathrm{CC}$

S3094

6, $\mathrm{CC}$

S3090

$7, \mathrm{CC}$

S3091

$8, \mathrm{CC}$

S3092

$9, \mathrm{CC}$

S3093

$11-1,65-70$

$12-1,109-112$

12-2, 99-104

$12-3,116-122$

$12-4,134-139$

$15-2,30-34$

21-4, 96-99

22-1, 126-129

$22-2,7-10$

$22-2,118-122$

22-3, 30-34

$22-3,110-114$

23-1, 68-71

23-1, 141-145

23-2, 74-77

24-1, 107-109

24-2, 13-16

24-2, 86-88

$25-1,120-123$

S3095

S3096

S3097

S3098

S3099

S3100

S3102

S3103

S3105

S3104

S 3101

S3106

S3107

S3108

S3109

S 3110

S3111

S3112

S3113

S3203

S3114

$25-2,87-89$

$25-3,15-18$

S3204

S3115

$25-3,113-115$

S3205

$\begin{array}{ll}26-1,113-114 & \mathrm{~S} 3116 \\ 26-2,2-4 & \mathrm{~S} 3117\end{array}$

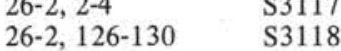

27-1, 92-95 S3119

27-2, 7-10 S3120

27-2, 121-125 S3121

Hole 328 (lat $49^{\circ} 48.67^{\prime} \mathrm{S}$, long $36^{\circ} 39.53^{\prime} \mathrm{W}$ )

6. $\mathrm{CC}$

S 3168

$7, \mathrm{CC}$

S3169

$8-2,125-128$

S3170

8-3, 31-33

S3171

9-1, 122-124

S 3172

9-2, 56-59

S 3173

9-4, 140-142

9-5, $125-128$

S3174

S3176

$9, \mathrm{CC}$

S3177

S 3178

10-6, $10-13 \quad$ S 3179

$10, \mathrm{CC} \quad \mathrm{S} 3180$

11-1, 0-2 S3181

$11-1,42-44 \quad \mathrm{~S} 3182$

11-3, 66-68 S3183

11-3, 136-138 S3184

$11-4,0-2 \quad \mathrm{~S} 3185$

$11-4,77-79 \quad$ S3186

11-5, 0-2 S3187

11-5, 91-93 S3188

11-6, 0-2 $\quad$ S3189

11-6, 69-71 S3190

$11, \mathrm{CC} \quad \mathrm{S} 3191$

12-1, 69-71 S3192

12-2, 20-22 S3193

12-6, 115-118 S3194

$12, \mathrm{CC} \quad \mathrm{S} 3195$ 
TABLE 1 - Continued

\begin{tabular}{ll}
\hline $\begin{array}{c}\text { Sample } \\
\text { (Interval in cm) }\end{array}$ & Sample No. \\
\hline Hole 328B & \\
$6-6,47-51$ & S 3196 \\
$7-1,5-9$ & S 3197 \\
$7-2,28-31$ & S3198 \\
$7-3,104-106$ & S3199 \\
$7-4,112-116$ & S3200 \\
$7-5,30-33$ & S3201 \\
$7-6,4-7$ & S3202
\end{tabular}

Hole 249 (lat $29^{\circ} 56.99^{\prime} \mathrm{S}$, long $36^{\circ} 04.62^{\prime} \mathrm{E}$ )

$\begin{array}{ll}23-5,50-52 & \text { S } 3242 \\ 24-1,55-57 & \text { S } 3243 \\ 25-1,105-107 & \text { S } 3244 \\ 25-2,50-52 & \text { S } 3245 \\ 26-1,56-58 & \text { S } 3246 \\ 26-2,102-103 & \text { S3247 } \\ 27-1,125-127 & \text { S } 3248 \\ 27-3,90-92 & \text { S } 3249 \\ 28-2,75-77 & \text { S } 3250 \\ 28-3,75-77 & \text { S } 3251 \\ 29-1,50-52 & \text { S } 3252 \\ 30-2,50-52 & \text { S } 3253 \\ 30-3,78-80 & \text { S } 3255 \\ 30-4,38-40 & \text { S } 3254 \\ 31-1,98-100 & \text { S } 3256 \\ 31-2,75-77 & \text { S } 3257 \\ 31-3,80-82 & \text { S } 3258 \\ 32-1,118-120 & \text { S } 3259\end{array}$

Hole 330 , lat $50^{\circ} 55.19^{\prime} \mathrm{S}$, long $46^{\circ} 53.00^{\prime} \mathrm{W}$ )

\section{3-2, 103-105}

4-1, 86-88

4-2, 131-133

5-1, 143-146

5-2, 91-95

5-3, 137-139

6-1, 96-98

6-2, 19-21

6-3, 44-47

6-4, 111-113

6-5, 9-11

6-6, 17-19

$7-1,123-126$

7-2, 55-59

$7-3,126-128$

$7-4,94-97$

$7-5,25-27$

$7-6,147-149$

8-2, 106-108

8-3, 116-119

$8-4,98-100$

9-2, 88-90

$10-1,129-134$

$10-2,130-132$

11-1, 94-96

$11-2,109-112$

$11-3,32-35$

$11-4,42-46$

$11-5,126-128$

11-6, 30-33

12-3, 133-135

$12-4,52-54$

$12-5,139-141$

$12-6,120-122$

$13-2,31-33$

$12-3,129-132$

$13-4,4-6$

$14-2,32-34$
TABLE 1 - Continued

\begin{tabular}{lc}
\hline $\begin{array}{c}\text { Sample } \\
\text { (Interval in cm) }\end{array}$ & Sample No. \\
\hline \multicolumn{2}{l}{ Hole $330-$ Continued } \\
$14-3,27-29$ & S3128 \\
$14-4,148-150$ & S3127 \\
\hline
\end{tabular}

\section{S. cingulatus \\ Trichodinium castanea (Deflandre) \\ Early Cretaceous-Cores 22-27}

Cores 13 to 21 were barren of plant microfossils. Core 22 contains:

1) Spores and pollen

Alisporites grandis (Cookson)

A. similis (Balme)

Caytonipollenites pallidus (Reissinger)

Ceratosporites equalis Cookson and Dettmann

Cicatricosisporites australiensis (Cookson)

Cyatheacidites sp.

Cyathidites australis Couper

C. minor Couper

Classopollis classoides Pflug

Dictyotosporites speciosus Cookson and Dettmann

Ephedripites sp. (see Plate 2, Figure 19)

Gleicheniidites circinidites

Laevigatosporites ovatus

Lycopodiumsporites reticulumsporites (Rouse)

Microcachryidites antarcticus Cookson

Staplinisporites caminus (Balme)

Tsugaepollenites trilobatus (Balme)

T. dampierii (Balme)

2) Microplankton

Chlamydophorella nyei Cookson and Eisenack

Chytroeisphaeridia sp.

Cleistosphaeridium sp.

Cribroperidinium orthoceras (Eisenack)

Gonyaulacysta helicoidea (Eisenack and Cookson)

Meiourogonyaulax sp.

Odontochitina operculata (O. Wetzel)

Prolixosphaeridium sp.

Spinidinium boydii Morgan

Spiniferites sp. cf. S. ramosus

$S$. wetzeli (Deflandre)

Tenua sp. cf. T. hystrix Eisenack

Core 23 is similar but dinoflagellates are less common (10\%-15\%).

$H$. wetzeli is very common. Belodinium aff. $B$. dysculum Cookson and Eisenack first appears in Core 24 together with Broomea sp., Cymatiosphaera (thickwalled form), Crassosphaera sp., gen. and sp. indet. (see Plate 14, Figure 18), Muderongia simplex Alberti, and ?Pterodinium magnoserratum Cookson and Eisenack.

In Core 25, Section 2, Oligosphaeridium complex (White) dominates the dinoflagellate assemblages. Several other undescribed species appear in this assemblage.

In Core 25, Section 3, ?Cyclonephelium sp. (see Plate 13, Figure 4) dominates the assemblage, and Classopollis is the dominant pollen. T. trilobatus is very common and Pterospermella is common. Species which 
appear in this core include Meiourogonyaulax sp. aff. $M$. bulloidea (Cookson and Eisenack), Gonyaulacysta sp. aff. G. hadra Sargeant, Canningia sp. cf. C. minor Cookson and Hughes, Prolixosphaeridium sp. cf. $P$. parvispinum (Deflandre). Core 26 is more or less similar to Core 25 but includes Dichadogonyaulax sp., Ephedripites sp. (see Plate 2, Figure 23), Tenua hystrix and large ?Canningia (see Plate 16, Figure 3). Other species include Pareodinia sp., B. aff. $B$. dysculum, and Tanyosphaeridium sp. Core 27 is essentially similar to Core 26 but does have a cf. Scriniodinium sp.

\section{Correlation and Age}

\section{Cores 5-9}

The presence of Deflandrea sp. A (see Plate 5, Figure 5), a species recorded from the Paleocene of DSDP Site 214 in the Indian Ocean (Harris, 1974, pl. 4, fig. 1-3), and Wetzeliella homomorpha, a common late Paleocene to early Eocene species of southern Australia and Europe (see charts of Drugg and Stover, 1975), indicates a late Paleocene age. Other species are of little use in determining a more precise age, but are consistent with an early Tertiary aspect.

\section{Cores 10-12}

The assemblages from this interval are similar to those of Site 328 which carry Odontochitina porifera Cookson (see later section) but are of a younger aspect. In particular, the presence of Eisenackia crassitabulata and Odontochitina sp. A suggests a Maestrichtian age.

\section{Cores 22-27}

In terms of spore-pollen and dinoflagellate cyst assemblages, these cores relate closely to Australian and Indian Ocean assemblages described by Cookson and co-workers and Wiseman and Williams (1974), but nevertheless have a large component of apparently endemic forms.

Cores 22 and 23 contain few distinctive forms to permit a firm dating, but the presence of Dictyotosporites speciosus in Core 22 is indicative of an Early Cretaceous (Aptian-Neocomian) or Late Jurassic age. The other cores contain a distinctive dinoflagellate assemblage with Belodinium sp. cf. B. dysculum, Broomea sp., and Muderongia simplex indicating an early Aptian to Neocomian age. Wiseman and Williams (1974) report similar assemblages from Neocomian sediments in the Indian Ocean.

\section{Environments of Deposition}

Early Tertiary sediments in Cores 5-9 contain a significant component of terrestrially derived organic matter (spores, pollen, tracheids) and probably indicate shallow shelf environments modified by terrestrial runoff. The change between these cores and those of the Late Cretaceous (Cores 10-12) is significant. Terrestrial components in the Late Cretaceous sediments are rare and the organic matter is almost entirely derived from marine microplankton. The sediments are of deeper water aspect.

Cores 13 to 21 are barren of plant microfossils. This may be due to several causes such as unfavorable con- ditions during sedimentation (oxidizing environments) or postdepositional oxidation of organic matter.

Cores 22-27 contain abundant organic matter with a very high proportion of woody matter, spores, and pollen. The sapropelic nature of the sediments and the preponderance of terrestrially derived components points to a near-shore marine (perhaps barred euxinic) environment.

\section{SITE 328}

Samples examined are from Cores 6 to 12 at Hole 328 and Cores 6 and 7 from Hole 328B. Many samples are barren. In general the assemblages are very sparse and always dominated by dinoflagellates although plant debris including tracheids and cuticle are often abundant.

One sample only from Core $6, \mathrm{CC}$ was virtually barren except for very dark brown microfossils, probably pyritized diatoms. Likewise Core 7 is virtually barren but it does have these very rare fossils. An operculum from Odontochitina porifera is present, but this could be reworked. This species is common in the lower sections of the hole. This core may be of similar age to Core 6.

All samples from Cores 8 and 9 except for the corecatcher sample from Core 9 are barren. The latter sample contains very rare Haloragacidites harrisii (Couper), Cyathidites sp., Milfordia homeopunctata McIntyre, Podocarpidites ellipticus, Laevigatosporites major (Cookson), and a Cleistosphaeridium sp. with abundant tracheid and cuticle material.

The assemblage is more characteristic of the Tertiary than of the Cretaceous, but it may be contaminated from sections higher in the well.

The first definitive Late Cretaceous assemblage occurs in Core 10, Section 1. The fossils are very rare but include a very characteristic undescribed form (see Plate 12, Figure 1). Terrestrial components are rare but include Cyathidites australis and Caytonipollenites pallidus.

The distribution of species in the four lower cores are tabulated in Table 2. Samples from Cores 6 and 8 at Site 328B were barren. Microplankton are in excess of $95 \%$ of the assemblages.

\section{Correlation and Age}

The problems of correlation and age of this section are not so very different from those of Site 327. Again there is a very strong component of Australasian and particularly Western Australian forms. The stratigraphic and geographic ranges of many of these are poorly known.

The following assignment of ages is based in part on the ages given by Cookson and Eisenack $(1960,1962)$ and Evans (1966). These ages will undoubtedly be modified as more evidence becomes available.

Cores 10 and 11 contain Nothofagidites sp., Proteacidites sp., and Nelsoniella spp. and are of Campanian to Maestrichtian age. The presence of Dinopterygium cladoides and Trichodinium castanea indicates an age older than Campanian, and Actinotheca fenestrata and Nelsoniella spp. are no older than Turo- 
TABLE 2

Distribution of Species, Hole 328B

\begin{tabular}{ccccc}
\hline & \multicolumn{5}{c}{ Core } \\
Species & 10 & 11 & 12 & 7 \\
\hline
\end{tabular}

Spores and Pollen

Caytonipollenites pallidus

Classopollis classoides

Cyathidites australis

Gleicheniidites circinidites

Laevigatosporites ovatus

Matonisporites $\mathrm{sp}$.

Nothofagidites sp.

Podocarpidites sp.

Proteacidites sp. indet.

Microplankton

Actinotheca aphroditae Cookson and Eisenack

Aiora fenestrata (Deflandre and Cookson)

Canningia sp. 22/1, 17/3

Cannosphaeropsis tutulosa Cookson and Eisenack

Conosphaeridium striatoconus (Deflandre and Cookson)

Coronifera sp. cf. $c$. sp.

Cleistophaeridium spp.

(Plate 12, Figures 4, 7)

Cribroperidinium orthoceras

Cyclonephelium sp.

Deflandrea sp. aff. D. echinoidea Cookson and Eisenack

Dinopterygium cladoides Deflandre

Gen. et. sp. indet. (Plate 12 , Figure 1)

Gillinia hymenophora

Hexagonifera glabra Cookson and Eisenack

H. vermiculata Cookson and Eisenack

Hystrichodinium sp.

Hystrichosphaeridium sp. (Plate 12, Figures 14, 17)

?Leptodinium sp. (Plate 21, Figure 5)

Membranosphaera sp.

Nelsoniella aceras Cookson and Eisenack

Nelsoniella semireticulata Cookson and Eisenack

Odontochitina cribropoda Deflandre and Cookson

O. porifera Cookson

?Spinidinium sp.

Spiniferites cingulatus

$S$. cingulatus aff. granulatus (Clarke and Verdier

Spiniferites ramosus

Trichodinium castanea (Deflandre)

Xenikoon australis

Cookson and Eisenack

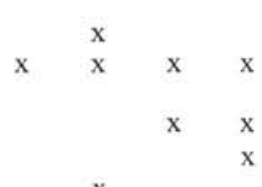

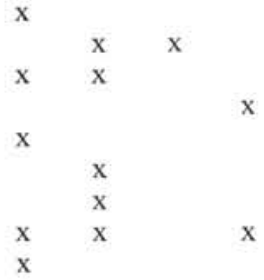

$\mathrm{X}$

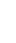
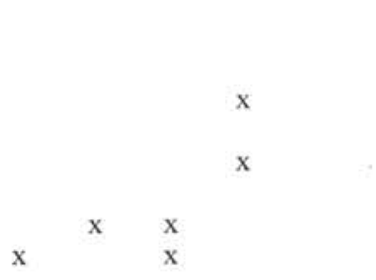

$\mathrm{x}$

$\mathrm{x}$

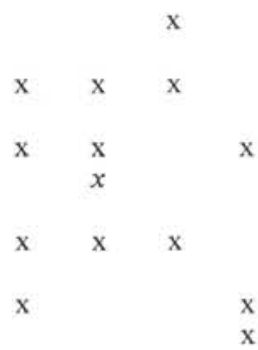

$\mathrm{x}$

$\mathrm{x} \quad \mathrm{x}$

x $\quad \mathrm{x}$

$\mathrm{x} \quad \mathrm{x}$

$\begin{array}{lll} & \mathrm{x} & \mathrm{x} \\ \mathrm{x} & \mathrm{x} & \mathrm{x}\end{array}$

$x^{x} \quad x^{-1}$

$\mathrm{x}$

$\mathrm{x}$ nian (Millioud et al., 1975). Core 7 from Hole 328B is Turonian to early Senonian and is older than Core 12 at Site 328.

\section{Environment of Deposition}

Spores and pollen are generally rare in all cores but are less rare in Cores 10 and 11. An outer shelf or deeper environment is suggested.

\section{SITE 330}

\section{Observations}

Palynological investigations at this site extend from Core 3 to Core 15 of Hole 330 . All of these cores yielded abundant very well preserved organic remains though often of low diversity. Microplankton (dinoflagellate cysts, acritarchs, and tasmanitids) dominate assemblages of Core 3 but rapidly decline to much less than $1 \%$ in lower cores.

\section{Early Cretaceous-Cores 3 and 4}

Core 3 contains a dinoflagellate assemblage very similar to the Lower Cretaceous cores at Site 327. In particular it has Belodinium cf. B. dysculum, Muderongia simplex, Dingodinium cerviculum Cookson and Eisenack, Cribroperidinium orthoceras, and Apteodinium $\mathrm{cf}$. A. maculatum Eisenack and Cookson. Core 4 is less diverse, but Pyxidiella sp. (see Plate 5, Figure 1), Dingodinium sp. cf. D. alberti Sargeant, Psaligonyaulax apatelum Cookson and Eisenack, $S$. dictyotum, Chlamydophorella nyei, Nannoceratopsis sp. cf. $N$. pellucida Deflandre, and Endoscrinium luridum (Deflandre) are present. The terrestrial component of both cores is dominated by disaccate pollen.

\section{Late Jurassic - Cores 5-15}

In this section the dinoflagellate cysts become less frequent to rare in Cores 14 and 15 . Cores 5 to 7 carry a low frequency of dinoflagellate cysts which include Tenua sp., Pareodinia sp., Leptodinium mirabile Klement, Oligosphaeridium complex, Meiourogonyaulax spp., Chytroeisphaeridia sp., Dingodinium sp., Gonyaulacysta spp., and several species of Prasinophyceae-Cymatiosphaera spp. and Pterospermella spp. Spores and pollen are abundant, dominated by Tsugaepollenites spp. and in particular by $T$. trilobatus.

A biofacies change occurs between Cores 7 and 8 . Tasmanitids become very abundant at the expense of dinoflagellate cysts and acritarchs. Dinoflagellate cysts down to Core 14 include Endoscrinium luridum (Core 12), Nannoceratopsis pellucida (Core 11), Leptodinium spp., Gonyaulacysta spp., Tenua hystrix, and ?Valensiella sp. In the lowest sample (Core 15, Section 2) dinoflagellate cysts and tasmanitids are very rare, and woody matter dominates the residues.

Important spores and pollen occurring in Cores 5 to 15 include Contignisporites cooksonii (Balme) (Cores 15 and 11), Dictyotosporites complex Cookson and Dettmann (Core 11), Staplinisporites caminus (Balme) (Core 14), and Densoisporites velatus Weyland and Krieger (Core 13).

\section{Correlation and Age}

\section{Cores 3 and 4}

Core 3 is virtually identical to Core 24 at Site 327 and is therefore of Neocomian to early Aptian age. Core 3 
assemblages are indicative of a reworked Late Jurassic component-Endoscrinium luridum and Nannoceratopsis sp.-and an Early Cretaceous component-Dingodinium cf. D. albertii, Chlamydophorella nyei, and Pyxidiella $\mathrm{sp}$. The latter has been recorded by Habib (1972) from the Aptian to Barremian at DSDP Site 101 in the North Atlantic. Thus the available evidence indicates that Core 4 is of late Neocomian to early Aptian age.

\section{Cores 5-15}

The rarity of dinoflagellate cysts, the most useful microfossil group for subdividing this part of the sequence, restricts their biostratigraphic utility. Nevertheless species such as Nannoceratopsis sp. cf. $N$. pellucida and Endoscrinium luridum are Oxfordian to Kimmeridgian species.

Filatoff $(1975)$ has recently reviewed the literature on Jurassic microfloras, and it is possible to make some correlation with the section described here and those in the Perth Basin of Western Australia. The presence of Contignisporites cooksonii and Dictyotosporites complex indicates a correlation with Filatoff's (1975) Contignisporites cooksonii Oppel-zone which ranges in age from late Callovian to Oxfordian. The presence of Densoisporites velatus in Core 13, a species characteristic of the base of Filatoff's youngest zone, would favor an Oxfordian age.

Thus the evidence is consistent for an Oxfordian to Kimmeridgian age for these sediments. There is no evidence for them being older than Oxfordian and there appears to be a substantial time break between Cores 4 and 5 which is reflected in part by remanié Jurassic microfossils in Core 4.

\section{Environments of Deposition}

All sediments in this interval were deposited in nearshore marginal marine environments. The excellent preservation of the microfossils attests to reducing conditions during sedimentation with very little if any metamorphic effects (thermal or load) after deposition.

Section 2 of Core 15 shows the least marine influence with very rare dinoflagellate cysts. These together with tasmanitids become more common up section to Core 8 which might be described as a tasmanite or oil shale. Indeed the abundance of Tasmanites at this level contributed the free "oil" released on preparation of the samples. More open marine influence is apparent between Cores 7 and 5, but the terrestrial components comprise $90 \%$ or more of the assemblages.

Cores 3 and 4 were deposited under similar conditions to the Jurassic sediments, but dinoflagellate cysts are more abundant in Core 3. A low energy euxinic near-shore marine environment is indicated.

\section{RELATIONSHIP OF DSDP SITE 249 TO THE SOUTH ATLANTIC SITES}

DSDP Site 249 was drilled on the Mozambique Ridge east of Durban, and continental reconstructions would place this position close to the sites in the South Atlantic. Samples from Cores 23 to 32 were examined. Cores 23,24 , and 32 are barren of plant microfossils.
In the other cores there is a very strong biofacies and biostratigraphic similarity with the Early Cretaceous sediments sampled at Sites 327 and 330. In particular, Belodinium sp. cf. B. dysculum, Muderongia simplex, Apteodinium maculatum, and Dingodinium cerviculum occur throughout the sequence. Spores and pollen include very common Classopollis sp., Dictyotosporites complex, and Cicatricosisporites australiensis. Thus the age of these cores is Neocomian to early Aptian.

The similarities between assemblages from Site 249 and from Sites 327 and 330 do not contradict any hypothesis that these three sites were in the same region during the Early Cretaceous.

\section{CONCLUSIONS}

Sedimentation on the Falkland Plateau commenced in the Oxfordian with the deposition of very marginal marine sapropelic siltstones and clays. These conditions continued into the Aptian with a major hiatus between the Oxfordian-Kimmeridgian and the NeocomianAptian sediments. Increasing marine influence is reflected by increase in numbers and diversity of dinoflagellate cysts in this part of the sequence.

The Late Cretaceous and early Tertiary sediments are in marked contrast with these and are deep-water deposits. In the early Tertiary at Site 328 , however, there is evidence in the increase of abundance of terrestrial organic matter. There was either a shallowing or an increase in runoff from some land source.

The relationships of the floras, both terrestrial and marine, are strongly austral. In particular the marine components show marked similarity with those of Western Australia and the Indian Ocean rather than those from New Zealand and Antarctica (Wilson, 1967a, b, c, 1968, 1975; Haskell and Wilson, 1975). This implies a proto southern Atlantic circulation linked with that of the Indian Ocean rather than access to Pacific waters through the Antarctic Peninsula-Scotia Arc and Tierra del Fuego.

\section{ACKNOWLEDGMENTS}

I am grateful to several colleagues with whom I discussed these assemblages. Roger Davey, Roger Morgan, Rex Harland, Warren Drugg, and Harry Leffingwell have given their time in examining some of the assemblages and their comments have been appreciated. Roger Morgan read early drafts of the manuscript.

All material was provided by the National Science Foundation. The trustees of the British Museum (Natural History), London, allowed access to types and figured specimens of palynomorphs in their collections.

This paper is published with the permission of the Director of Mines, South Australia.

\section{REFERENCES}

Cookson, I.C. and Eisenack, A., 1960. Microplankton from Australian Cretaceous sediments: Micropaleontology, v. 6, p. 1-8.

- 1962. Additional microplankton from Australian Cretaceous sediments: Micropaleontology, v. 8, p. 485507.

Drugg, W.S. and Stover, L.E., 1975. Stratigraphic range charts of selected fossil dinoflagellates; selected Cenozoic 
dinoflagellates: Am. Assoc. Stratigraphic Palynologists Contrib., Ser. 4, p. 73-76.

Evans, P.R., 1966. Mesozoic stratigraphic palynology of the Otway Basin: Bur. Min. Res. Aust. Rec. 1966/69 (unpublished),

Filatoff, J., 1975. Jurassic palynology of the Perth Basin, Western Australia: Palaeontographica B, v. 154, p. 1-113.

Habib, D., 1972. Dinoflagellate stratigraphy Leg 11, Deep Sea Drilling Project. In Hollister, C.D., Ewing, J.I., et al., Initial Reports of the Deep Sea Drilling Project, Volume 11: Washington (U.S. Government Printing Office), p. $367-425$.

Harris, W.K., 1974. Palynology of Paleocene sediments at Site 214, Ninetyeast Ridge. In von der Borch, C.C., Sclater, J.G., et al., Initial Reports of the Deep Sea Drilling Project, Volume 22: Washington (U.S. Government Printing Office), p. 503-519.

Haskell, T.R. and Wilson, G.J., 1975. Palynology of Sites 280-284, DSDP Leg 29, off Southeastern Australia and western New Zealand. In Kennett, J.P., Houtz, R.E., et al., Initial Reports of the Deep Sea Drilling Project, Volume 29: Washington (U.S. Government Printing Office), p. 723-741.

Millioud, M.E., Williams, G.L., and Lentin, J.K., 1975. Stratigraphic range charts of selected fossil dinoflagellates. Selected Cretaceous dinoflagellates: Am. Assoc. Stratigraphic Palynologists Contrib., Ser. 4, p. 65-71.

Wilson, G.J., 1967a. Microplankton from the Garden Cove Formation, Campbell Island: New Zealand J. Bot., v. 5, p. 223-240. 1967b. Some new species of Lower Tertiary dinoflagellates from McMurdo Sound, Antarctica: New Zealand J. Bot., v. 5, p. 57-83.

1967c. Some species of Wetzeliella Eisenack (Dinophyceae) from New Zealand Eocene and Paleocene strata: New Zealand J. Bot., v. 5, p. 469-497.

, 1968. On the occurrence of fossil microspores, pollen grains and microplankton in bottom sediments of the Ross Sea, Antarctica: New Zealand J. Mar. Freshwater Res., v. 2, p. 381-389. 1975. Palynology of deep-sea cores from DSDP Site 275, southeast Campbell Plateau. In Kennett, J.P., Houtz, R.E., et al., Initial Reports of the Deep Sea Drilling Project, Volume 29: Washington (U.S. Government Printing Office), p. 1031-1035.

Wiseman, J.F. and Williams, A.J., 1974. Palynological investigation of samples from sites 259,261 and 263, Leg 27, Deep Sea Drilling Projects. In Veevers, J.J., Heirtzler, J.R., et al., Initial Reports of the Deep Sea Drilling Project, Volume 27: Washington (U.S. Government Printing Office), p. 915-924.

\section{PLATES}

Coordinates are from Zeiss photomicroscope No. 1953 from the Geological Survey of South Australia. Specimens have been photographed in normal transmitted light, phase contrast, and Nomarski interference contrast. 


\begin{abstract}
PLATE 1
All magnifications $\times 500$ unless otherwise stated

Figures 1,2 Cyathidites australis Couper.

1. Sample 330-13-2, 31-33 cm. Slide S3129/1, 18.4:103.8.

2. Sample 330-14-4, 148-150 cm. Slide S3127/1, 15.4:99.5.

Figure 3 Dictyophyllidites aff. D. crenatus Dettmann. Sample 330-13-2, 31-33 cm. Slide S3129/1, 16.4:117.2.

Figures 4, 5 Cibotiumsporites jurienensis (Balme). Sample 330-13-2, 31-33 cm. Slide S3129/1, 19.1:106.1.

Figure 6 Cyathidites minor Couper. Sample 330-13-2, 31-33 cm. Slide S3129/1, 9.4:121.1.

Figures $7,8 \quad$ Stereisporites spp.

7. Sample 327A-22-1, 126-129 cm. Slide S3103/1, 18.6:120.0.

8. Sample 330-14-4, 148-150 cm. Slide S3127/1, 16.6:122.8.

Figure 9 ?Osmundacidites sp. Sample 330-14-4, 148-150 cm. Slide S3127/1, 16.6:122.8.

Figures 10-14 Osmundacidites wellmanii (Couper).

10. Sample 330-14-4, 148-150 cm. Slide S3127/1, 19.0:103.8.

11. Sample 330-14-4, 148-150 cm. Slide S3127/1, 10.7:112.2.

12. Sample 330-13-2, 31-33 cm. Slide S3129/1, 17.2:133.0.

13. Sample 330-12-6, 120-122 cm. Slide S3130/1, 5.0:111.9.

14. Sample 330-13-2, 31-33 cm. Slide S3129/1, 11.8:101.1.
\end{abstract}

Figure $15 \quad$ Baculatisporites $\mathrm{sp}$. Sample 330-14-4, 148-150 cm. Slide S3127/1, 8.2:102.7.

Figures 16-20 Verrucosisporites spp.

16. Sample 330-14-4, 148-150 cm. Slide S3127/1, 6.0:106.2.

17, 18. Sample 330-12-6, 120-122 cm. Slide S3130/1, 7.5:105.1.

19. Sample 330-14-4, 148-150 cm. Slide S3127/1, 12.4:103.5.

20. Sample 330-14-4, 148-150 cm. Slide S3127/1, 16.1:99.1.

Figures 21, 22 Leptolepidites crassobalteus Filatoff.

21. Sample 330-14-4, 148-150 cm. Slide S3127/1, 12.2:123.2.

22. Sample 330-14-4, 148-150 cm. Slide S3127/1, 8.8:109.0.

Figure 23 Neoraistrickia truncatus (Cookson). Sample 327A-22-1, 126-129 cm. Slide S3103/1, 18.8:108.4.

Figure 24 Foveotriletes sp. Sample 327A-22-1, 126-129 cm. Slide S3103/1, 10.8:100.7.

Figure 25 ?Ischyosporites $\mathrm{sp}$. Sample 330-14-4, 148-150 cm. Slide S3127/1, 19.8:107.4.

Figure 26 Foveotriletes $\mathrm{sp}$. Sample 330-11-5, 126-128 cm. Slide S3135/1, 7.1:111.5.

Figure 27 ?Ischyosporites $\mathrm{sp}$. Sample 330-12-6, 120-122 cm. Slide S3130/1, 11.8:122.8.

Figures 28-30 Gleicheniidites circinidites (Cookson).

28. Sample 327A-22-1, 126-129 cm. Slide S3103/1, 14.5:108.7.

29. Sample 330-14-4, 148-150 cm. Slide S3127/1, 8.9:120.0.

30. Sample 330-14-4, 148-150 cm. Slide S3127/1, 19.1:104.3.

Figure 31 Camarozonosporites sp. Sample 330-14-4, 148-150 cm. Slide S3127/1, 7.9:96.7.

Figures 32-36 Trilites spp.

32. Sample 330-13-2, 31-33 cm. Slide S3129/2, 6.3:110.0.

33. Sample 330-13-2, 31-33 cm. Slide S3129/1, 11.9:106.2.

34. Sample 330-13-2, 31-33 cm. Slide S3129/1, 18.7:102.5.

35. Sample 330-13-2, 31-33 cm. Slide S3129/1, 10.7:116.1.

36. Sample 330-14-4, 148-150 cm. Slide S3127/1, 18.1:109.0.

Figures 37, 38 Lycopodiumsporites $\mathrm{sp}$.

37. Sample 330-14-4, 148-150 cm. Slide S3127/1, 6.8:111.0.

38. Sample 330-14-4, 148-150 cm. Slide S3127/1, 12.0:104.0.

Figure 39 Lycopodiumsporites sp. cf. rosewoodensis (de Jersey). Sample 330-14-4, 148$150 \mathrm{~cm}$. Slide S3127/1, 16.2:110.7. 


\section{PLATE 1}
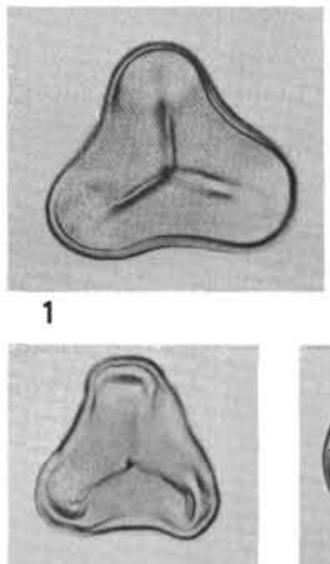

6

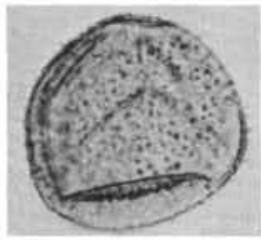

12

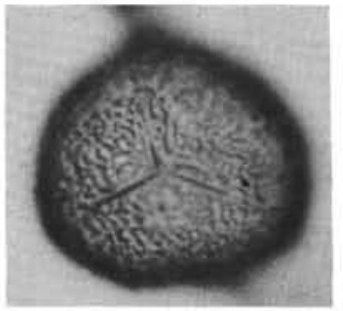

17

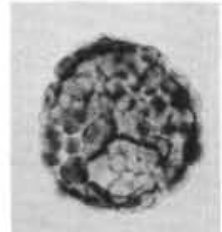

22

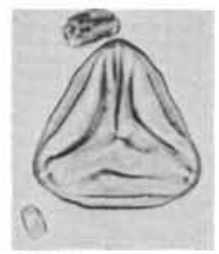

28

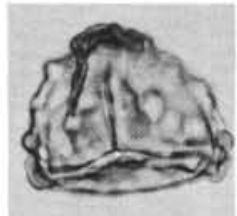

34

35

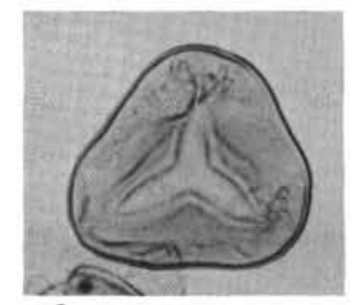

2

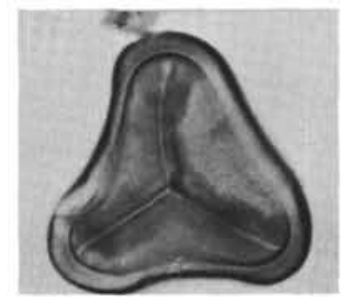

3

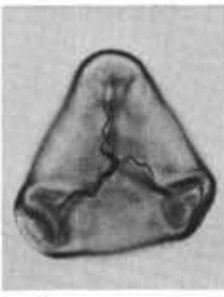

4

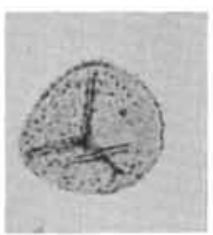

10

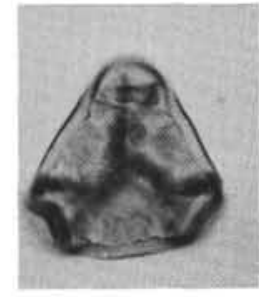

5

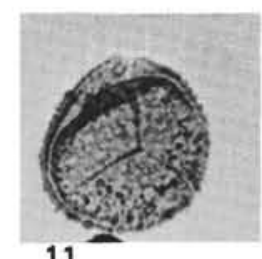

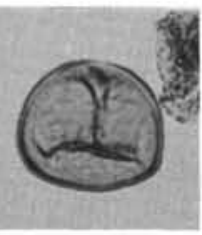

8

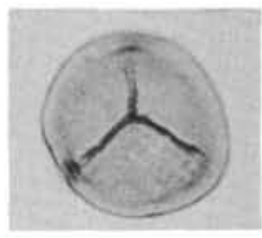

9

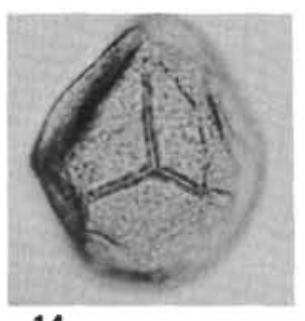

14

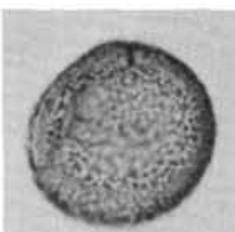

13

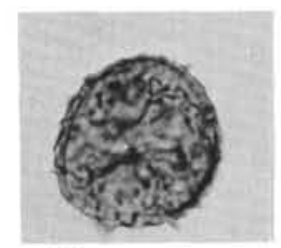

15
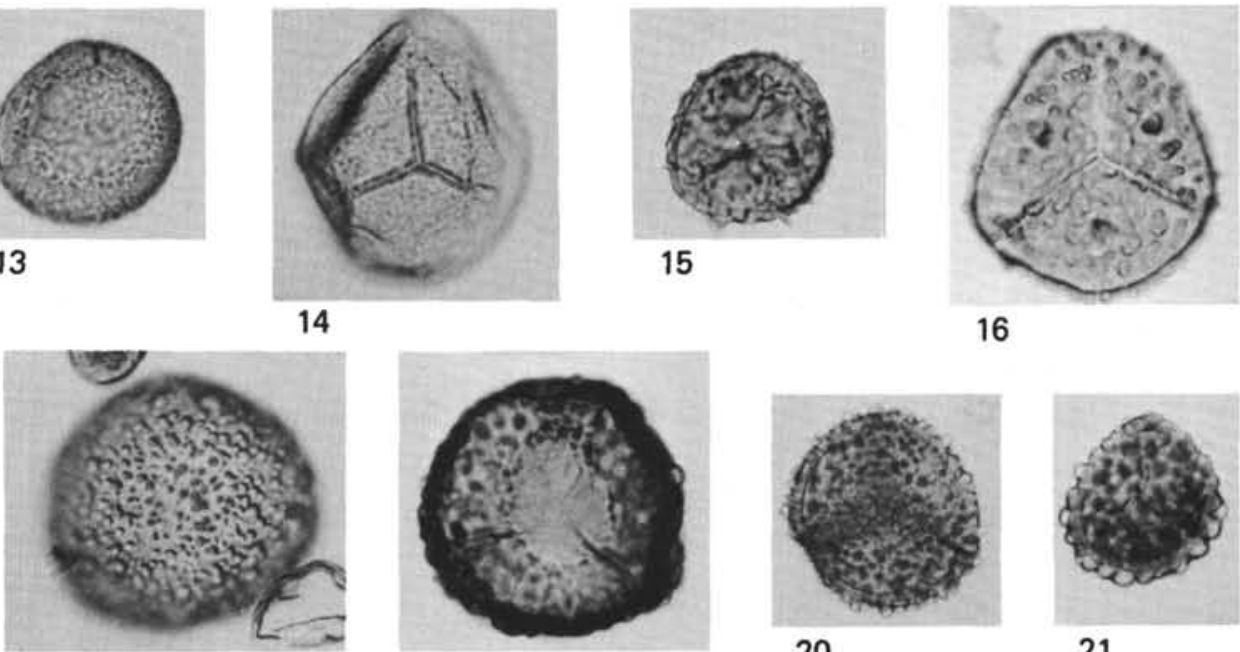

18

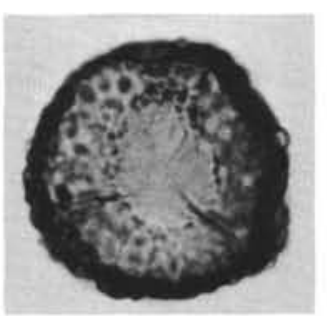

19

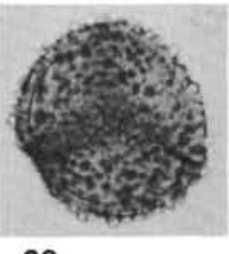

20

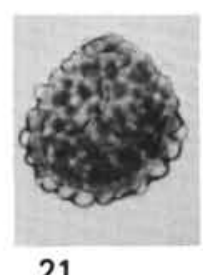

21

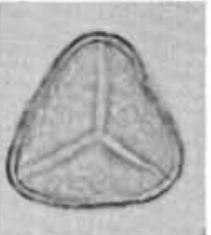

26

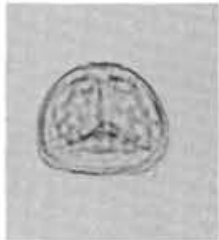

25

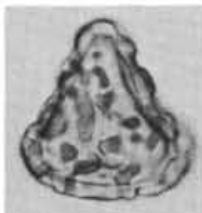

32

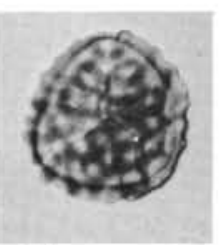

31

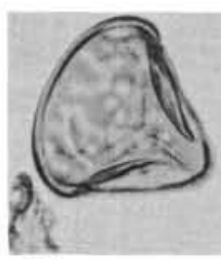

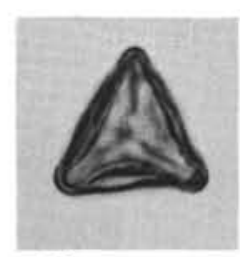

30

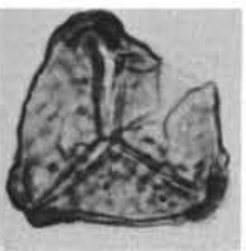

36

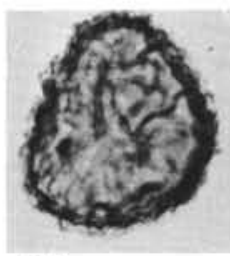

37

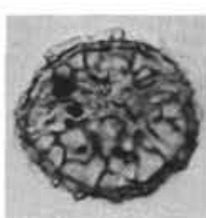

38
27
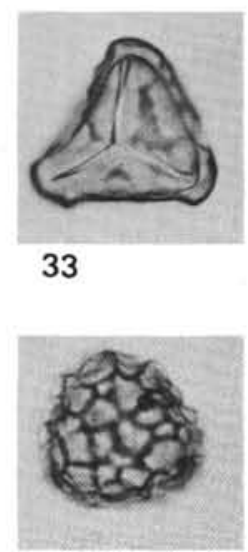

39 


\section{PLATE 2}

All magnifications $\times 500$ unless otherwise stated

Figure 1 Lycopodiumsporites reticulumsporites (Rouse). Sample 330-11-4, $42-46 \mathrm{~cm}$. Slide S3136/1, 20.8:100.0.

Figure 2 Sestrosporites pseudoalveolatus (Couper). Sample 330-14-4, 148-150 cm. Slide S3127/1, 16.5:109.1.

Figure 3 Matonisporites crassiangulatus (Balme). Sample 330-14-4, 148-150 cm. Slide S3127/1, 8.6:106.8.

Figures 4, 5 Ischyosporites sp. cf. I. punctatus Cookson and Dettmann.

4. Sample 330-14-4, 148-150 cm. Slide S3127/1, 7.0:111.2.

5. Sample 330-14-4, 148-150 cm. Slide S3127/1, 6.9:101.0.

Figure 6 Ischyosporites marburgensis de Jersey. Sample 330-12-3, 133-135 cm. Slide S3133/1, 13.2:103.2.

Figure 7 Ischyosporites crateris Balme. Sample 330-14-4, 148-150 cm. Slide S3127/1, 10.6:101.9.

Figure 8 Antulsporites saevus (Balme). Sample 330-5-1, 143-146 cm. Slide S3161/1, 6.4:107.0.

Figures 9, 10 Staplinisporites caminus (Balme).

9. Sample $330-14-4,148-150 \mathrm{~cm}$. Slide S3127/1, 10.0:97.3.

10. Sample 330-14-4, 148-150 cm. Slide S3127/1, 11.5:106.1.

Figure $11 \quad$ Contignisporites sp. cf. C. multimuratus Dettmann. Sample 330-14-4, 148$150 \mathrm{~cm}$. Slide S3127/1, 16.3:106.2.

Figure 12 Contignisporites cooksonii (Balme). Sample 330-11-6, 30-33 cm. Slide S3134/1, 9.9:114.3.

Figures 13,14 Densoisporites velatus Weyland and Krieger.

13. Sample 330-13-2, 31-33 cm. Slide S3129/1, 12.5:114.5.

14. Sample 330-13-2, 31-33 cm. Slide S3129/1, 4.8:127.5.

Figure 15 Cicatricosisporites australiensis (Cookson). Sample 327A-22-1, 126-129 cm. Slide S3103/1, 11.4:107.5.

Figure 16 Classopollis sp. Sample 330-14-4, 148-150 cm. Slide S3127/1, 10.5:115.6.

Figure 17 Dictyotosporites complex Cookson and Dettmann. Sample 330-11-5, 126$128 \mathrm{~cm}$. Slide S3135/1, 7.1:107.4.

Figures 18,19 Ephedripites $\mathrm{sp}$.

18. Sample 330-3-2, 103-105 cm. Slide S3157/2, 4.9:99.1.

19. Sample 330-3-2, 103-105 cm. Slide S3157/2, 6.5:113.2.

Figures 20, 21 Classopollis sp. Sample 330-13-2, 31-33 cm. Slide S3129/1, 18.2:107.3.

Figures 22, 23 Ephedripites spp.

22. Sample 327A-22-1, 126-129 cm. Slide S3103/1, 2.3:104.0.

23. Sample 330-4-2, 131-133 cm. Slide S3162/3, 5.4:94.9.

Figure 24 Ginkgocycadophytus sp. Sample 330-12-6, 120-122 cm. Slide S3130/1, 5.7:124.6. 


\section{PLATE 2}
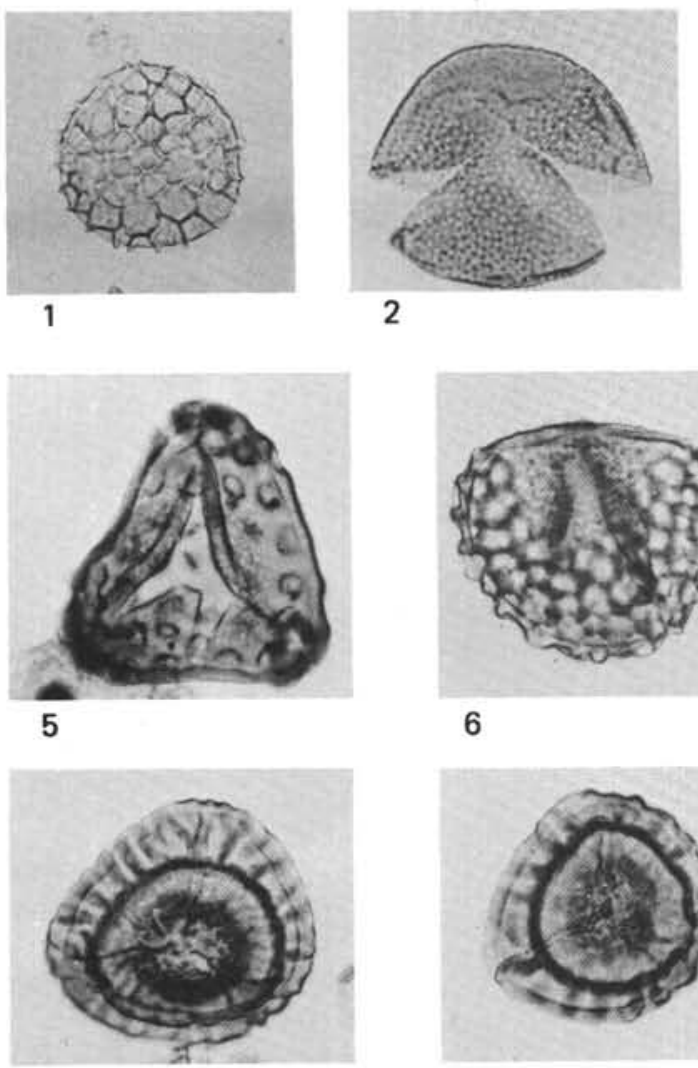

9

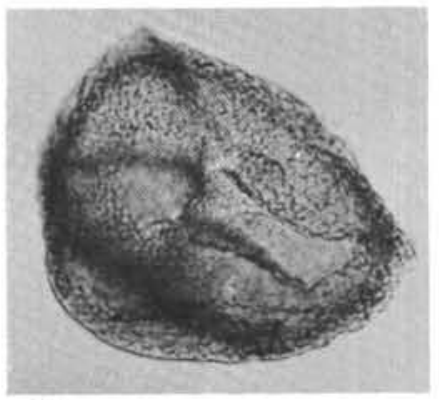

13

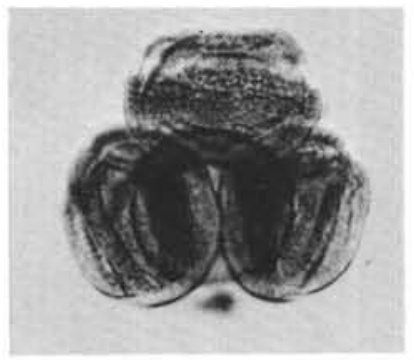

16

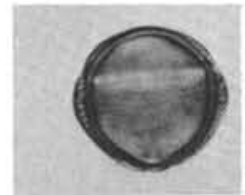

20

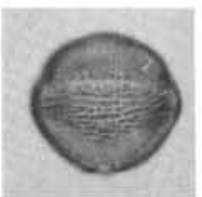

21
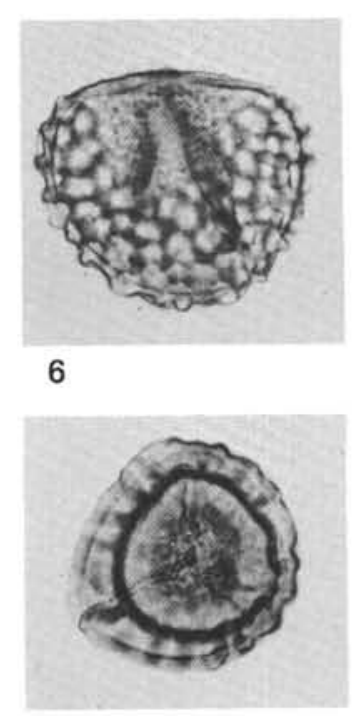

10

14
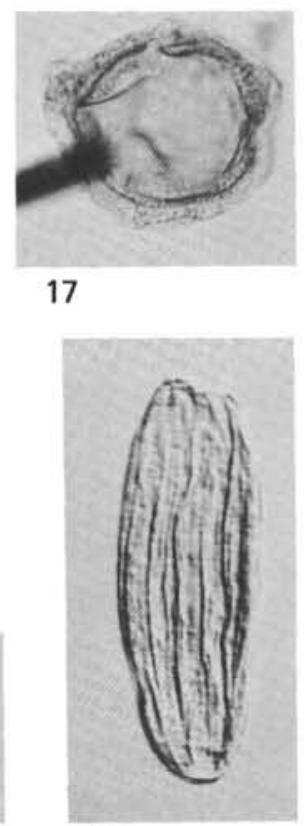

22

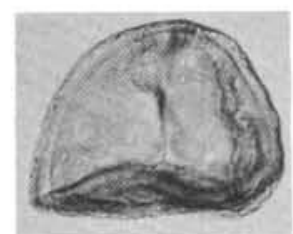

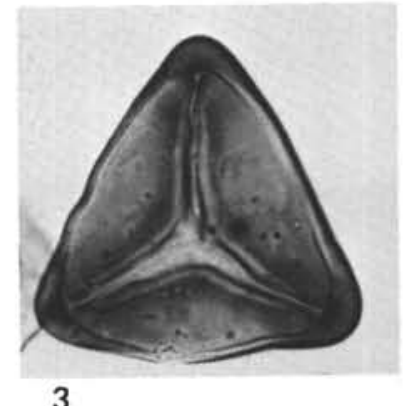
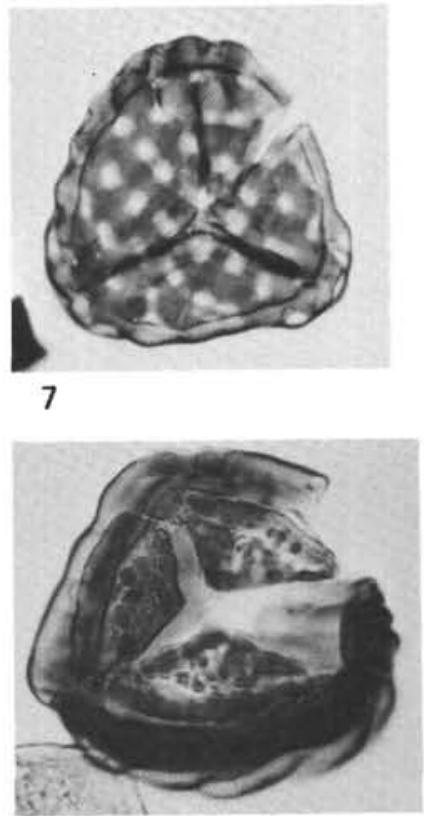

11

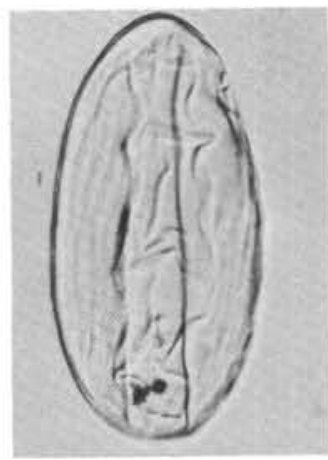

18

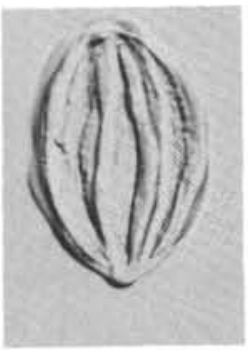

23
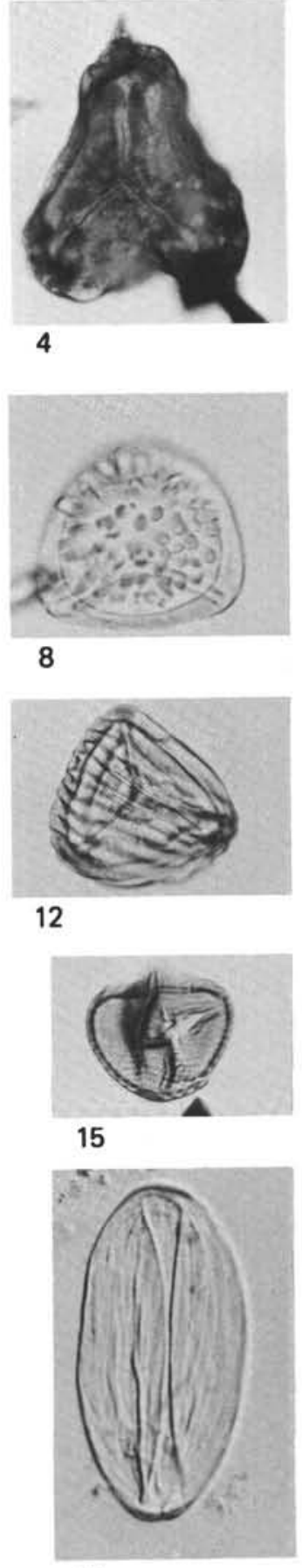

19 


\section{PLATE 3}

All magnifications $\times 500$ unless otherwise stated

Figure 1 Tsugaepollenites segmentatus (Balme). Sample $330-14-4,148-150 \mathrm{~cm}$. Slide S3127/1, 14.2:117.0.

Figures 2, $3 \quad$ Tsugaepollenites dampieri (Balme).

2. Sample $330-14-4,148-150 \mathrm{~cm}$. Slide S3127/1, 7.6:96.4.

3. Sample $330-14-4,148-150 \mathrm{~cm}$. Slide S3127/1, 7.9:98.6.

Figure $4 \quad$ Tsugaepollenites segmentatus (Balme). Sample 330-13-2, 31-33 cm. Slide S3129/1, 12.4:108.8.

Figure 5 Tsugaepollenites dampieri (Balme). Sample 330-14$4,148-150 \mathrm{~cm}$. Slide S3127/1, 12.8:123.3.

Figure 6 Tsugaepollenites trilobatus (Balme). Sample 330$14-4,148-150 \mathrm{~cm}$. Slide S3127/1, 10.5:110.6.

Figure 7 Callialasporites turbatus (Balme). Sample 330-126, 120-122 cm. Slide S3130/1, 10.9:124.6.

Figure $8 \quad$ Callialasporites turbatus (Balme). Sample 330-144, 148-150 cm. Slide S3127/1, 11.4:102.8.

Figures 9, 10 Tsugaepollenites trilobatus (Balme).

9. Sample 330-14-4, 148-150 cm. Slide S3127/1, 8.2:106.0.

10. Sample $330-14-4,148-150 \mathrm{~cm}$. Slide S3127/1, 11.6:113.7.

Figures 11, 12 Callialasporites turbatus (Balme).

11. Sample 330-14-4, $148-150 \mathrm{~cm}$. Slide S3127/1, 7.3:100.6.

12. Sample 330-14-4, $148-150 \mathrm{~cm}$. Slide S3127/1, 19.2:100.2.

Figure 13 Dictyotosporites complex Cookson and Dettmann. Sample 330-11-1, 94-96 cm. Slide S3139/4, 6.9:103.4. 
PLATE 3

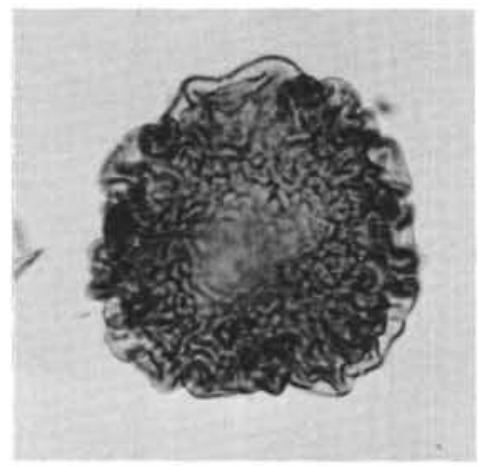

1

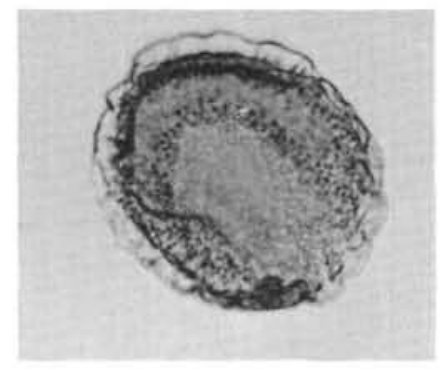

4
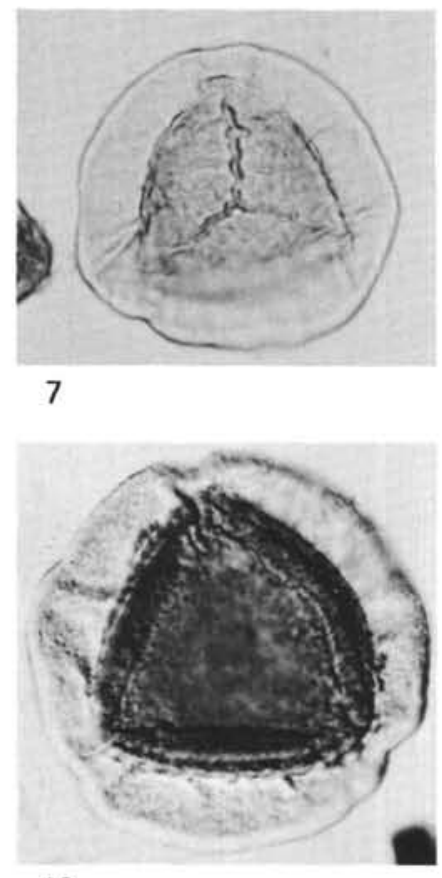

10

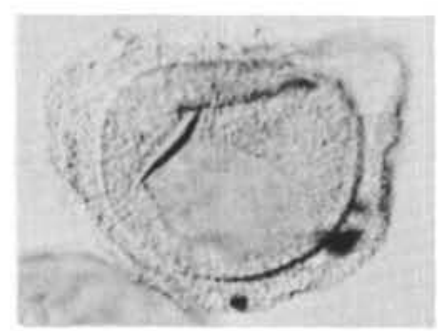

13
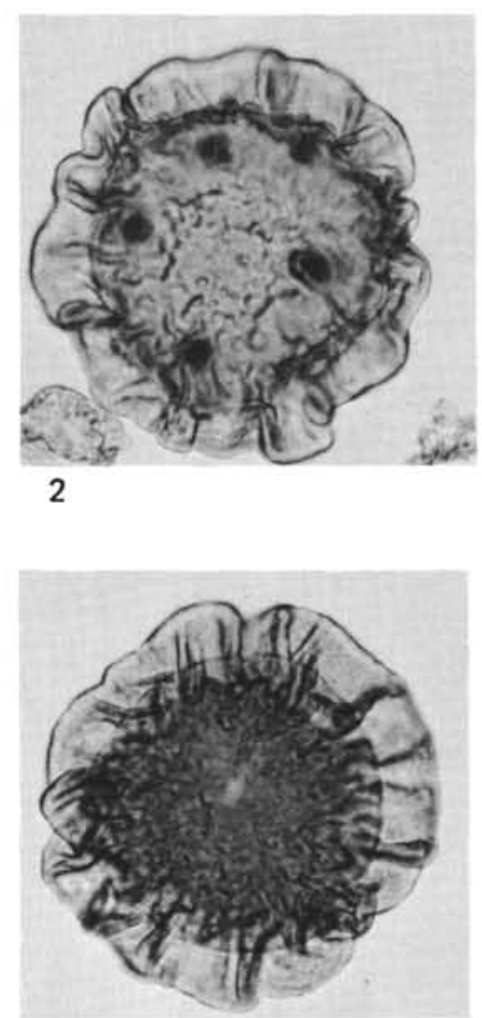

5

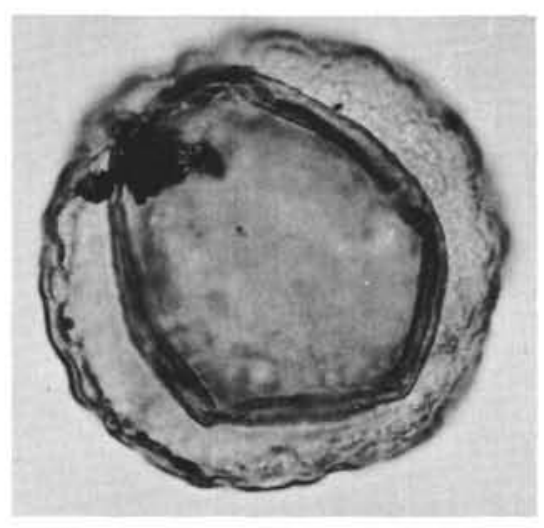

8

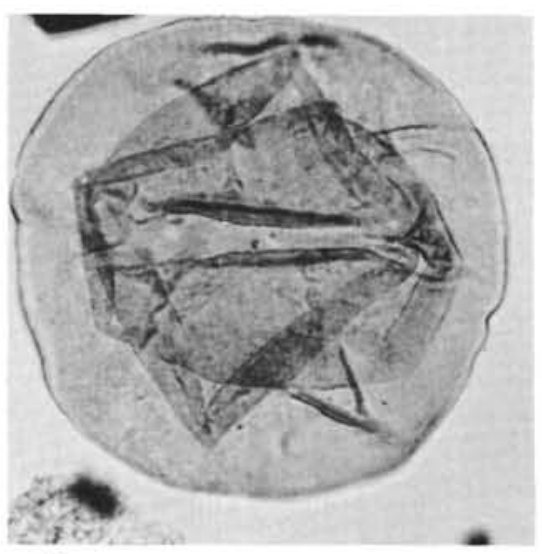

11

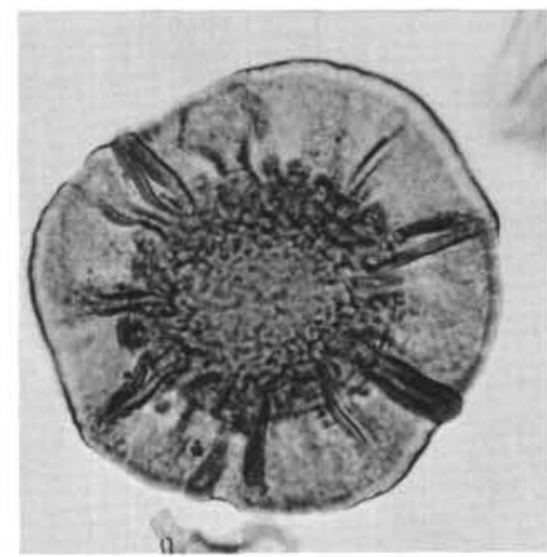

3

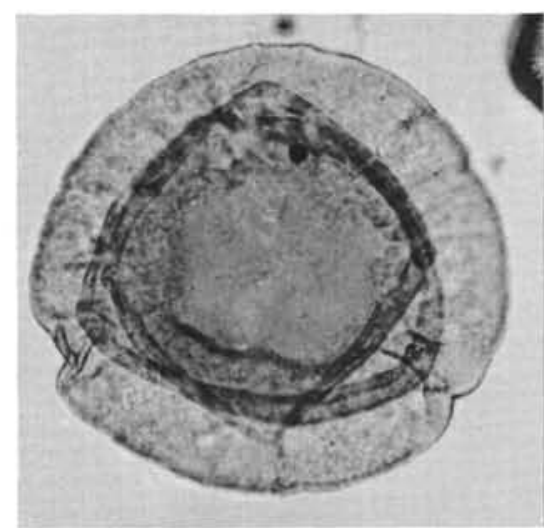

6

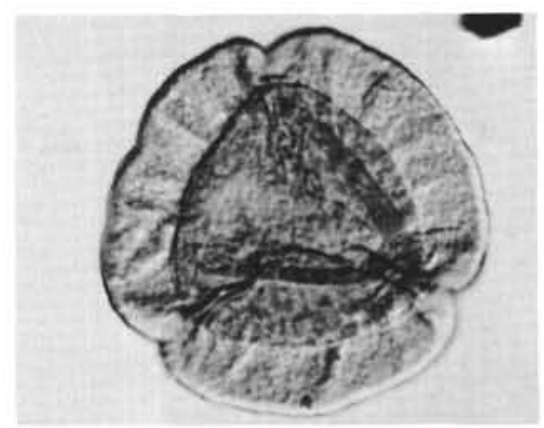

9

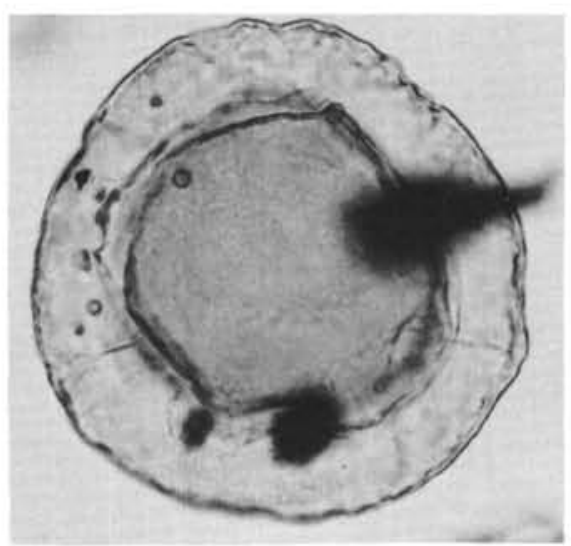

12 
All magnifications $\times 500$ unless otherwise stated

Figure $1 \quad$ Podocarpidites ellipticus (Cookson). Sample 330-32, 103-105 cm. Slide S3157/2, 10.7:123.2.

Figure 2 Pinuspollenites globosaccatus Filatoff. Sample 33012-6, 120-122 cm. Slide S3130/1, 16.9:107.0.

Figure $3 \quad$ Alisporites sp. cf. A. grandis Cookson. Sample 3303-2, 103-105 cm. Slide S3157/2, 14.2:109.3.

Figure $4 \quad$ Pinuspollenites globosaccatus Filatoff. Sample 3303-2, 103-105 cm. Slide S3157/2, 11.9:123.6.

Figure $5 \quad$ Pinuspollenites parvisaccatus (de Jersey). Sample 330-3-2, 103-105 cm. Slide S3157/2, 13.8:104.8.

Figure $6 \quad$ Alisporites similis (Balme). Sample 330-3-2, 103$105 \mathrm{~cm}$. Slide S3157/2, 10.8:106.7.

Figure $7 \quad$ Sp. indet. Sample 330-13-2, 31-33 cm. Slide S3129/1, 15.0:117.2.

Figure 8 Pinuspollenites sp. Sample 330-3-2, 103-105 cm. Slide S3157/2, 15.4:125.2.

Figures 9, 10, Microcachryidites sp. 14

9. Sample 330-14-4, 148-150 cm. Slide S3127/1, 21.0:124.1.

10, 14. Sample 330-3-2, 103-105 cm. Slide $\mathrm{S} 3157 / 2,12.0: 122.5$.

Figure $11 \quad$ Podocarpidites ellipticus (Cookson). Sample 33012-6, 120-122 cm. Slide S3130/1, 19.2:121.8.

Figure 12 Podocarpidites sp. Sample 330-3-2, 103-105 cm. Slide S3157/2, 12.2:122.3.

Figure 13 Microcachryidites antarcticus Cookson. Sample 330-14-4, 148-150 cm. Slide S3127/1, 10.7:114.6.

Figure $15 \quad$ Alisporites sp. Sample 330-3-2, 103-105 cm. Slide S3157/2, 10.8:105.1.

Figure $16 \quad$ Alisporites similis (Balme). Sample 330-12-6, 120 $122 \mathrm{~cm}$. Slide S3130/1, 16.2:124.1.

Figure 17 Alisporites sp. Sample 330-12-6, 120-122 cm. Slide S3130/1, 5.4:100.0.

Figure 18 Microcachryidites sp. Sample 330-12-6, 120-122 cm. Slide S3130/1, 6.1:99.8.

Figure 19 Caytonipollenites pallidus (Reissinger). Sample 330-13-2, 31-33 cm. Slide S3129/1, 8.6:109.6.

Figure 20 Sp. indet. Sample 330-7-2, $55-59 \mathrm{~cm}$. Slide S3159/1, 5.1:127.4.

Figure 21 Sp. indet. Sample 330-12-6, 120-122 cm. Slide S3130/1, 15.7:101.6.

Figure 22 Gen. et sp. indet. Sample 330-10-1, 129-134 cm. Slide S3141/1, 13.0:131.0. 
PLATE 4
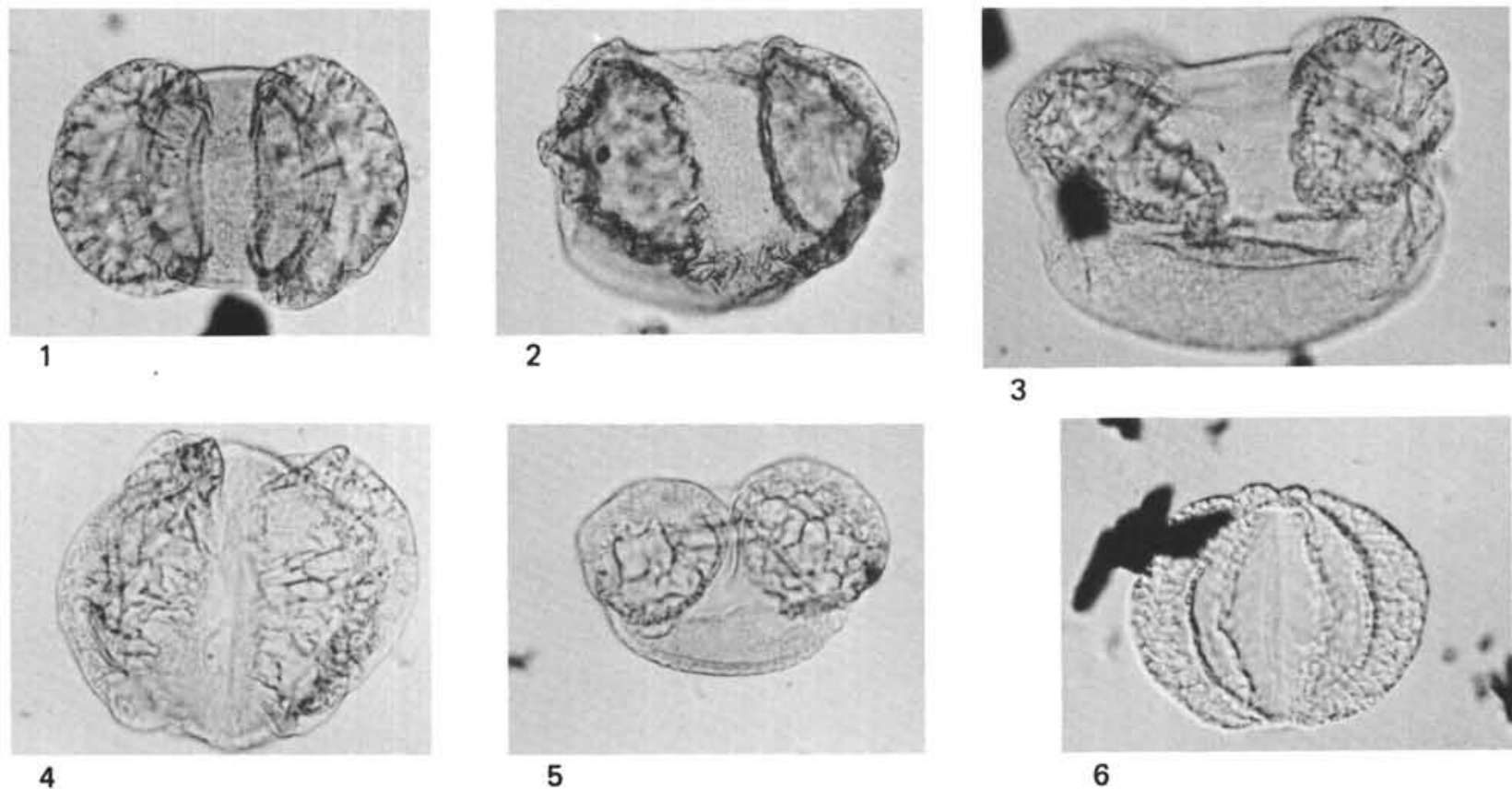

3
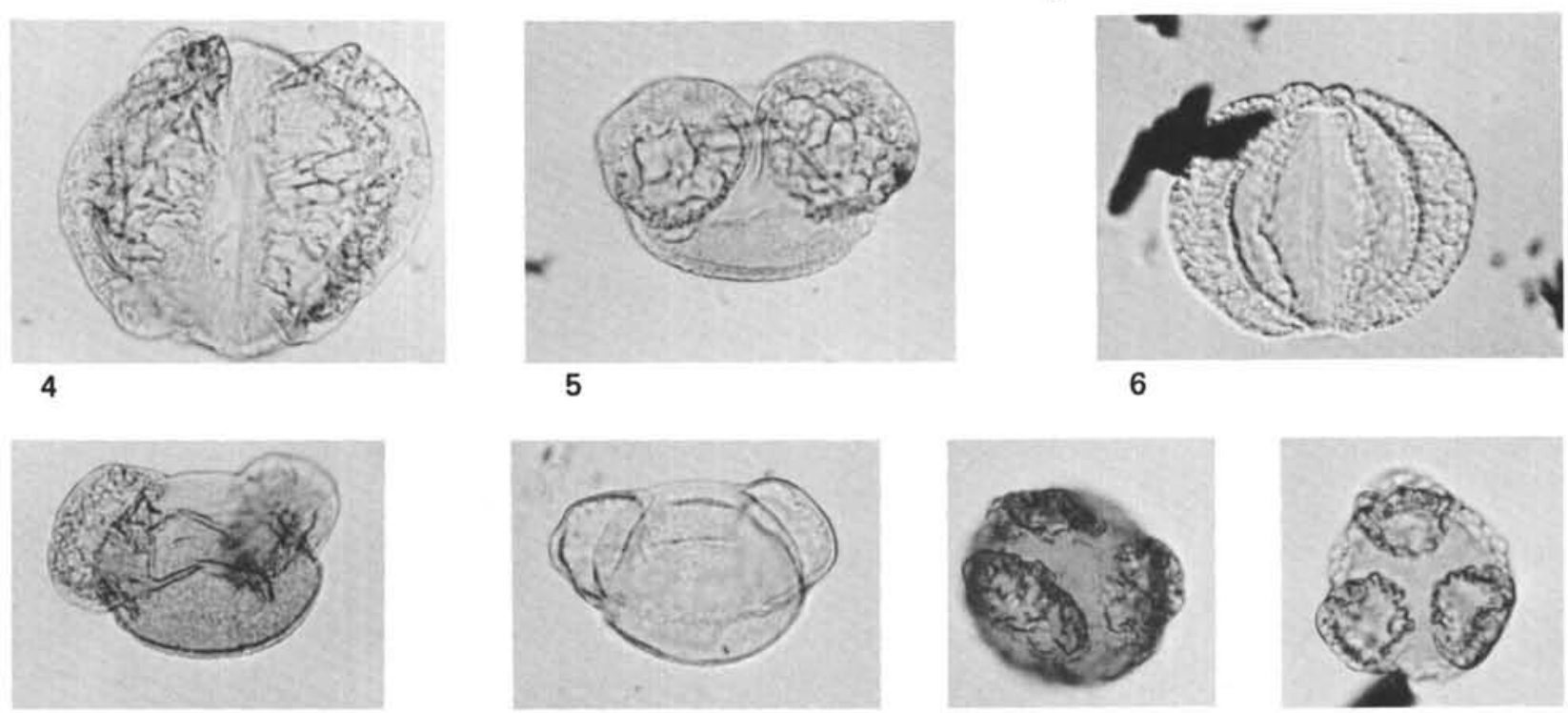

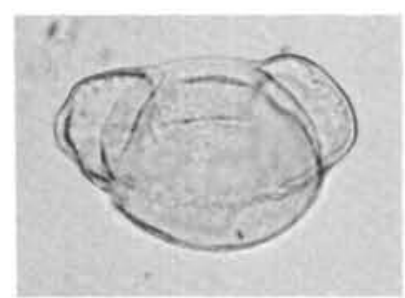

8

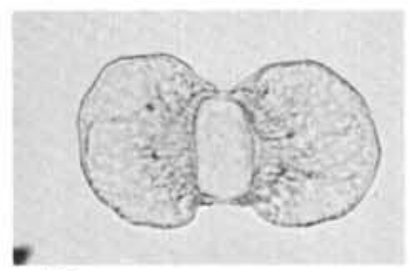

12

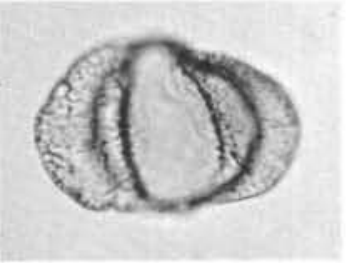

16

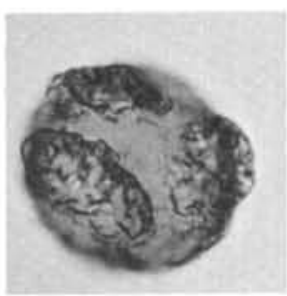

9

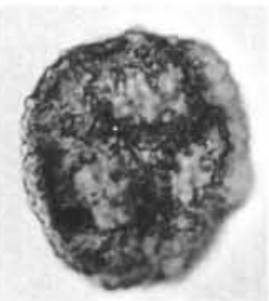

13

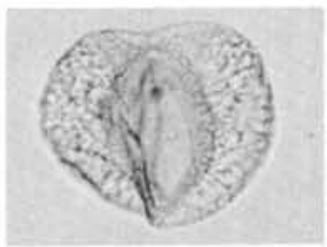

17
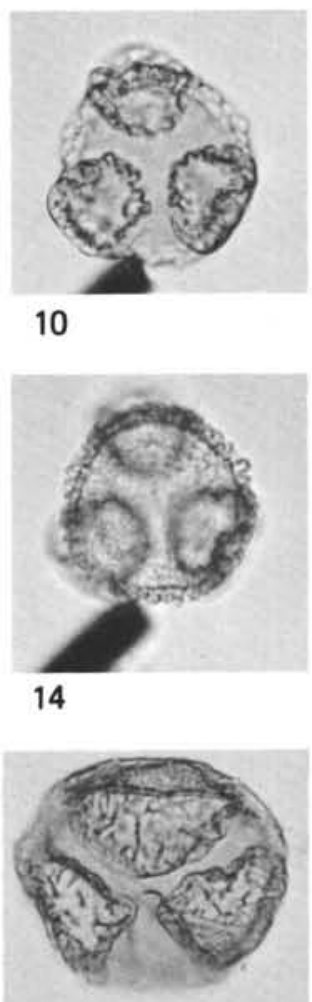

18

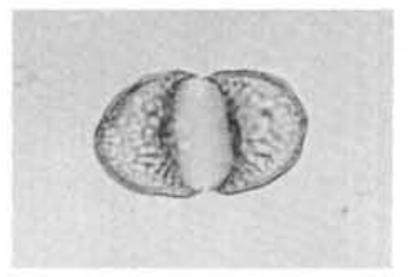

19
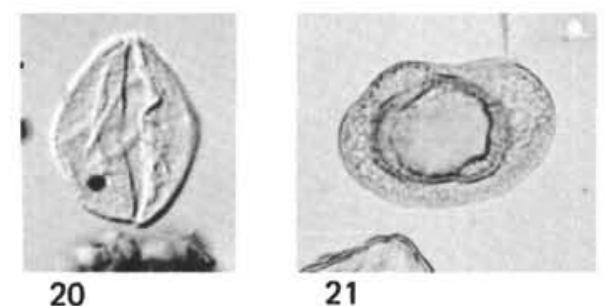

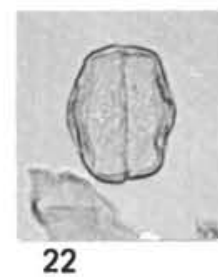


PLATE 5

All magnifications $\times 500$ unless otherwise stated

Figures 1, 2, $4 \quad$ Pyxidiella sp. of Habib, 1972.

1. Sample 327 A-22-1, 126-129 cm. Slide S3103/1, 2.6:104.1.

2. Sample 327A-22-1, 126-129 cm. Slide S3103/1, 15.0:114.0.

4. Sample 327 A-22-2, 7-10 cm. Slide ST3105/11, 15.8:111.9.

Figures 3,6 Deflandrea sp.

3. Sample 327A-12-2, $99-104 \mathrm{~cm}$. Slide ST 3097/2, 105.1:6.6.

6. Sample 327A-12-2, 99-104 cm. Slide ST3097/4, $8.7: 105.3$.

Figure 5 Deflandrea sp. A. Sample 327A-9, CC. Slide ST3093/4, 14.4:110.4.

Figures 7,8 Xenikoon australis Cookson and Eisenack.

7. Sample 327A-9, CC. Slide ST3093/3, 12.2:110.0

8. Sample 327A-8, CC. Slide ST3092/7, 13.2:104.6.

Figures 9,11 Deflandrea acuminata Cookson and Eisenack.

9. Sample 327A-8, CC. Slide ST3092/6, 16.5:102.4.

11. Sample 327A-8, CC. Slide ST3092/5, 12.7:103.3.

Figures 10,12 Sp. indet.

10. Sample 330-12-6, 120-122 cm. Slide S3130/1, 10.7:99.4.

12. Sample $330-13-2,31-33 \mathrm{~cm}$. Slide S3129/1, 18.4:106.1.

Figure $13 \quad$ Nelsoniella semireticulata Cookson and Eisenack. Sample 328-11-3, 66-68 cm. Slide ST3183/3, 16.8:106.1. 
PLATE 5

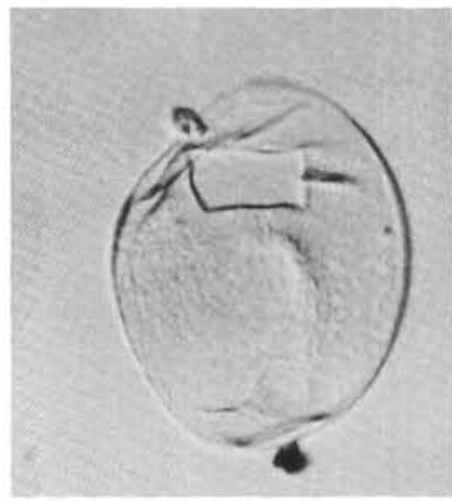

1

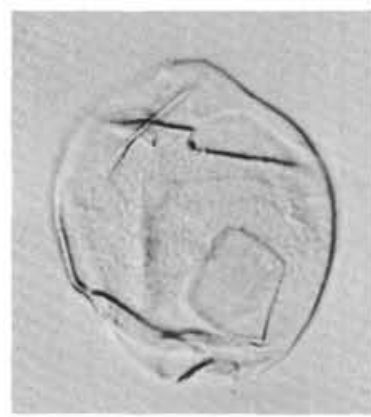

4

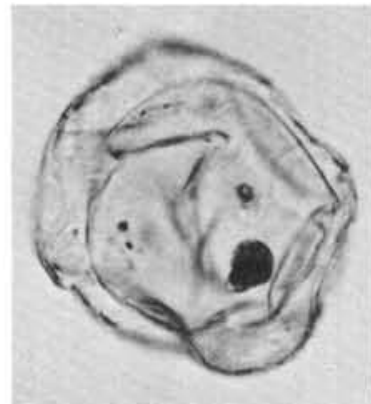

7

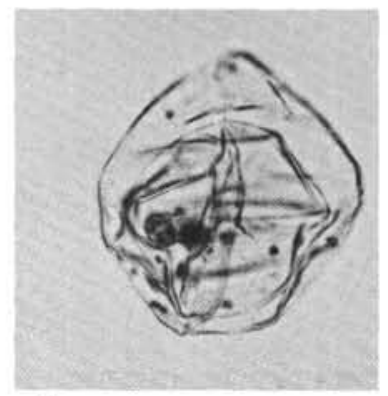

8

2
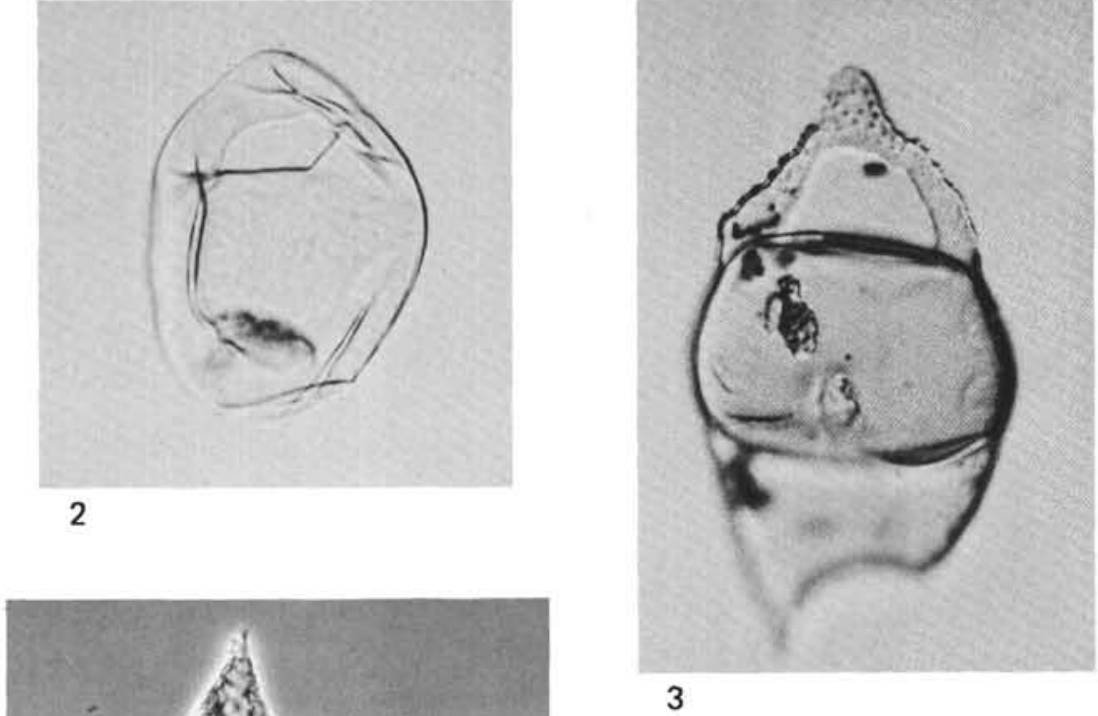

3
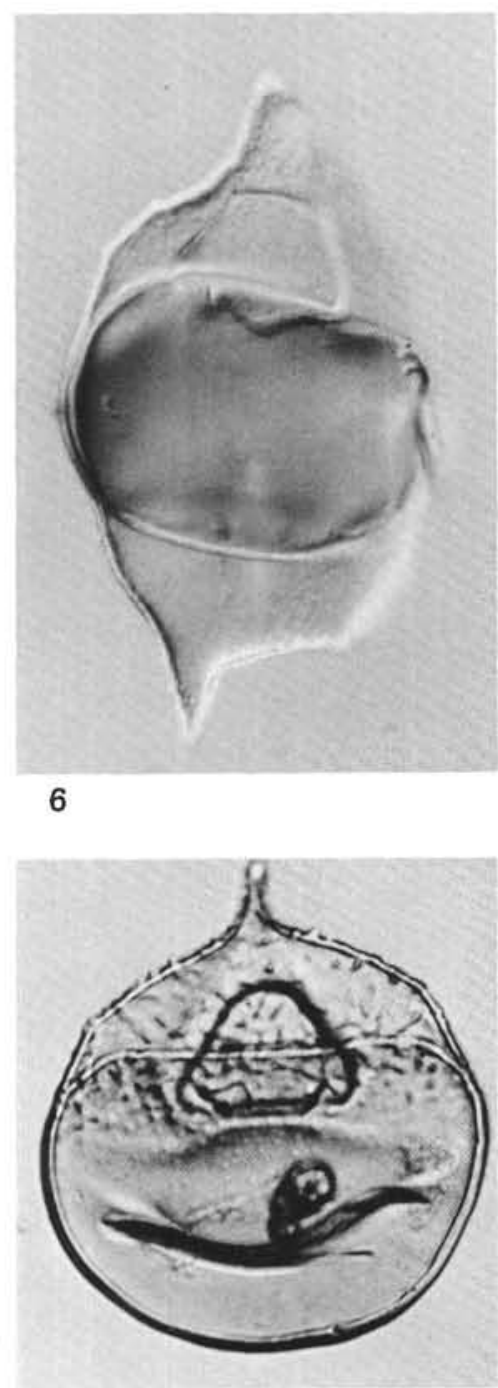

13 


\section{PLATE 6}

All magnifications $\times 500$ unless otherwise stated

Figures 1,2 Nelsoniella semireticulata Cookson and Eisenack. Sample 328-11-1, $42-44 \mathrm{~cm}$. Slide ST3182/1, 13.9:107.2. High and low focus.

Figure 3 Nelsoniella aceras Cookson and Eisenack. Sample 328-11-1, $42-44 \mathrm{~cm}$. Slide ST3182/5, 105.1:12.3.

Figure 4 Palaeoperidinium sp. Sample 327A-8. CC. Slide ST3092/8, 10.0:105.1.

Figure $5 \quad$ Palaeoperidinium sp. Sample 327A-9, CC. Slide ST3093/14, 13.6:102.0.

Figures 6,7 Gen. et sp. indet.

6. Sample 327A-22-1, 126-129 cm. Slide S3103/1, 13.9:97.1.

7. Sample 327 A-22-1, 126-129 cm. Slide S3103/1, 14.5:101.9.

Figures 8,9 Palaeoperidinium sp. Sample 327A-9, CC. Slide ST3093/1, 11.1:103.2. High and low focus.

Figure $10 \quad$ Palaeoperidinium sp. Sample 327A-9, CC. Slide ST3093/13, 15.8:106.4.

Figures 11-14 Spinidinium boydii Morgan.

11. Sample 327A-22-1, 126-129 cm. Slide S3103/1, 2.6:110.0.

12, 13. Sample 327A-22-1, 126-129 cm. Slide S3103/1, 22.2:115.4. High and low focus.

14. Sample 327A-22-1, 126-129 cm. Slide S3103/1, 12.8:132.2.

Figures 15,16 Deflandrea sp. cf. D. echinoide Cookson and Eisenack.

15. Sample 327 A-22-1, $126-129 \mathrm{~cm}$. Slide $3103 / 1$, 2.7:98.4.

16. Sample 327 A-22-2, 7-10 cm. Slide ST3105/5, 10.2:106.9. 
PLATE 6

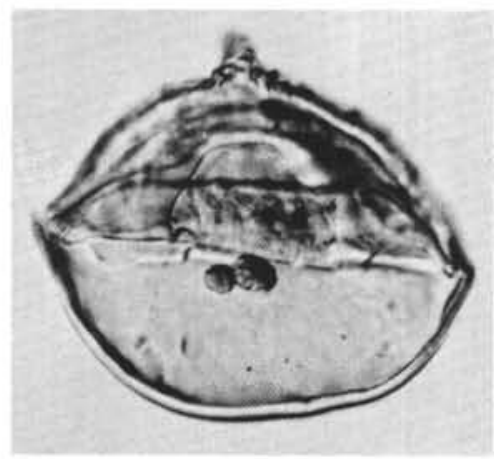

1

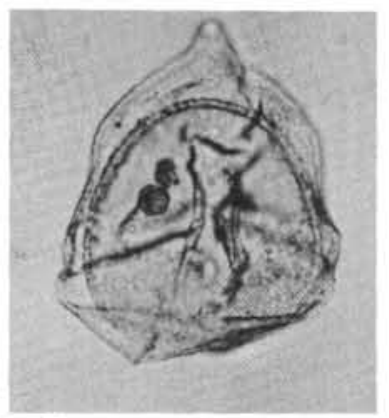

4

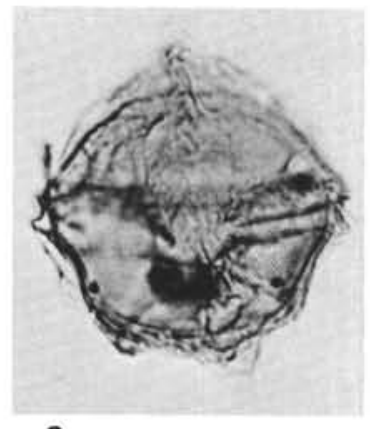

8

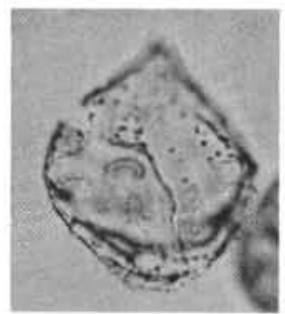

12

9

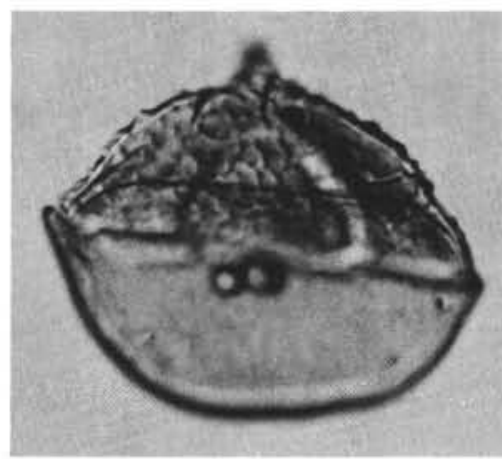

2

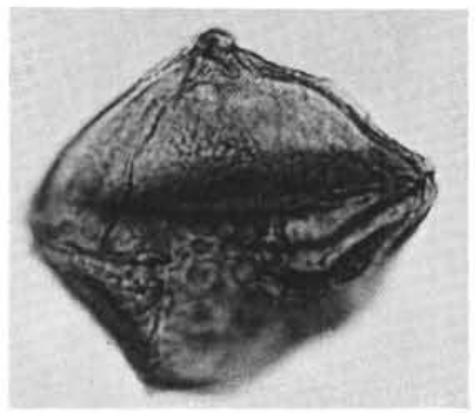

5
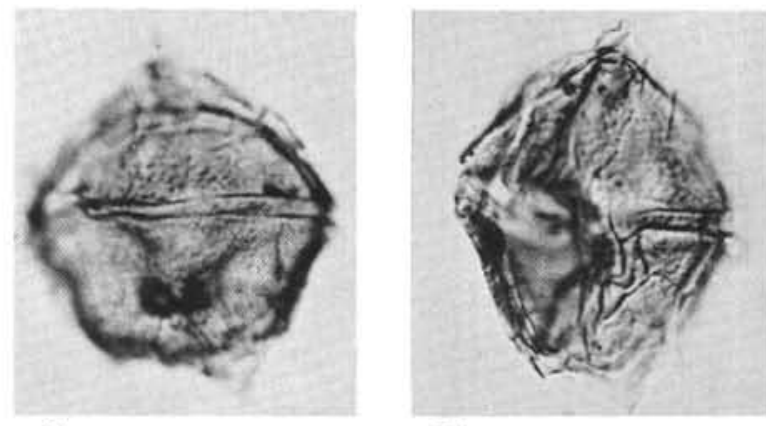

10

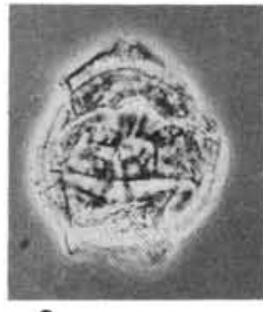

6

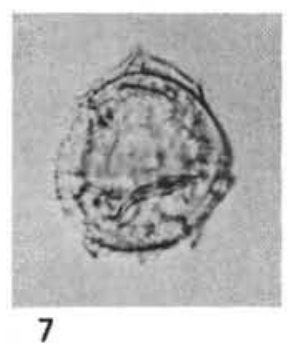

7

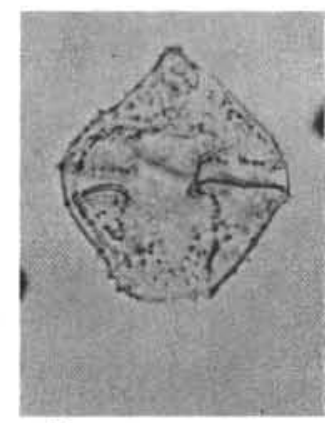

11

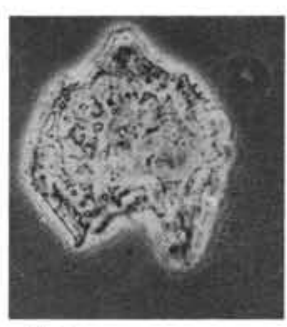

16 
PLATE 7

All magnifications $\times 500$ unless otherwise stated

Figures 1,2 Gonyaulacysta sp. Sample 330-3-2, 103-105 cm. Slide S3157/2, 6.1:100.0.

Figure 3 Cribroperidinium orthoceras (Eisenack). Sample $328-12-1,69-71 \mathrm{~cm}$. Slide ST3192/8, 11.2:109.6.

Figures 4, 5 Trichodinium castanea (Deflandre).

4. Sample 327A-11-1, 65-70 cm. Slide ST3095/5, 10.9:107.4.

5. Sample 327A-11-1, 65-70 cm. Slide ST3095/2, $110.8: 6.9, \times 300$.

Figure 6 Criboperidinium orthoceras (Eisenack). Sample 328-12-1, 69-71 cm. Slide ST3192/6, 12.8:103.0.

Figures 7, 8 Trichodinium castanea (Deflandre). Sample 327A11-1, 65-70 cm. Slide ST3095/6, 10.4:104.7.

Figures 9, 10 Gonyaulacysta helicoidea (Eisenack and Cookson). Sample 330-3-2, 103-105 cm. Slide S3157/2, 15.6:116.9. 
PLATE 7

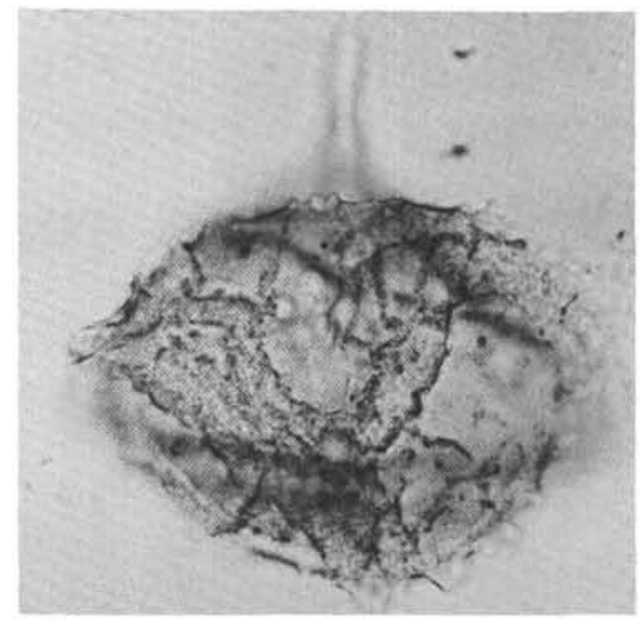

1
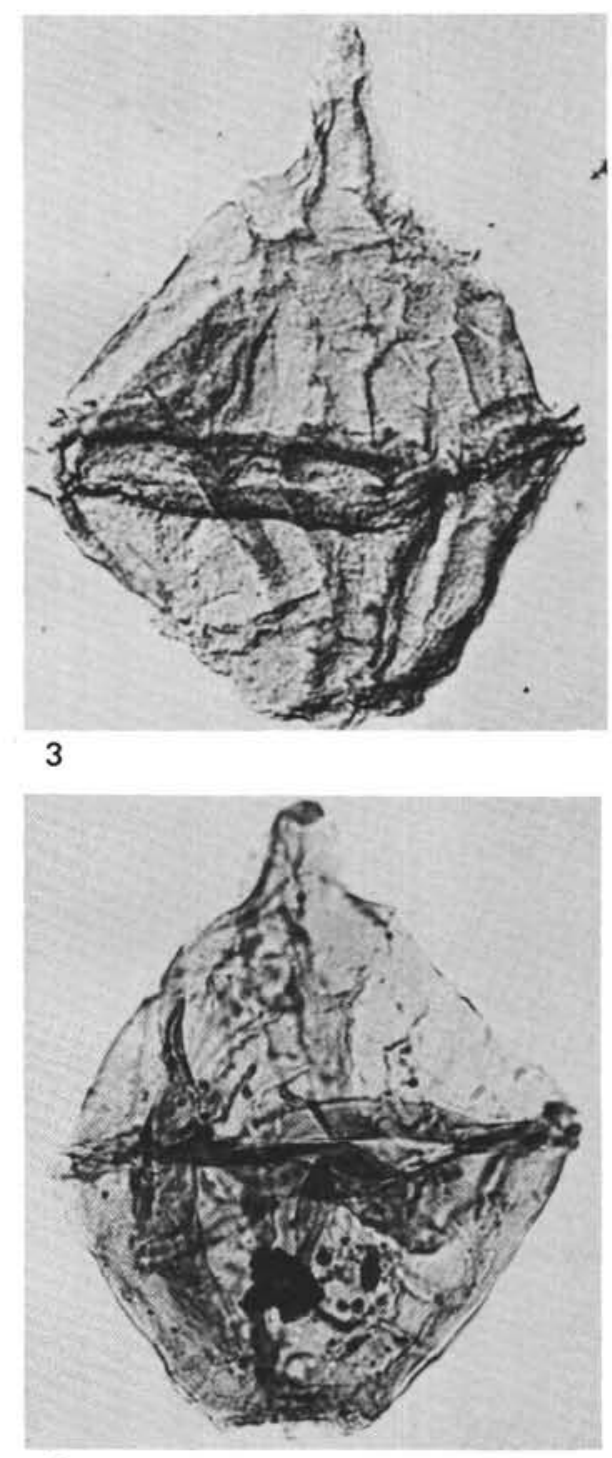

6

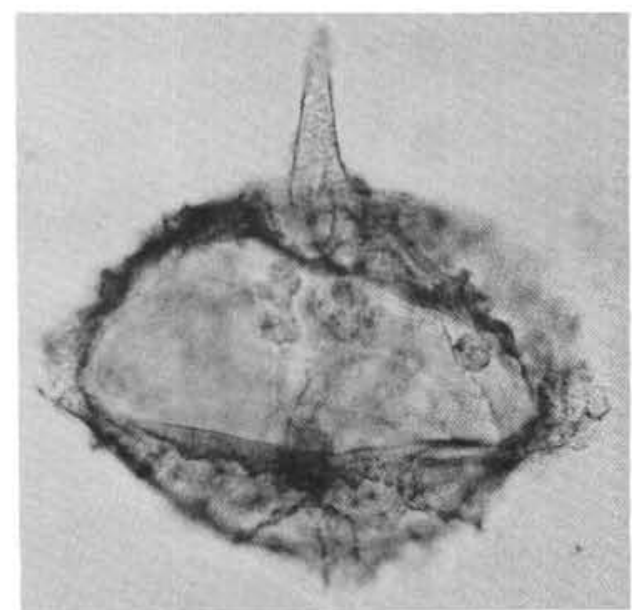

2
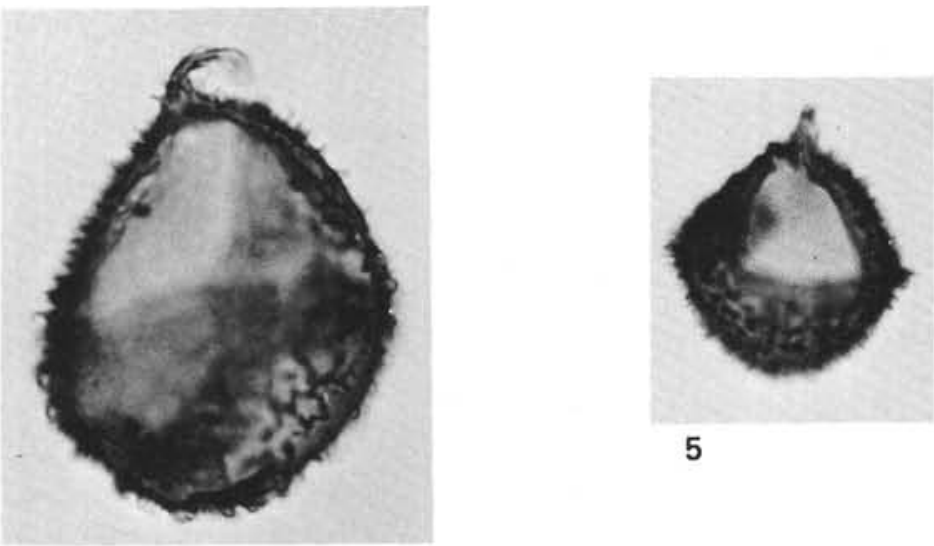

5

4

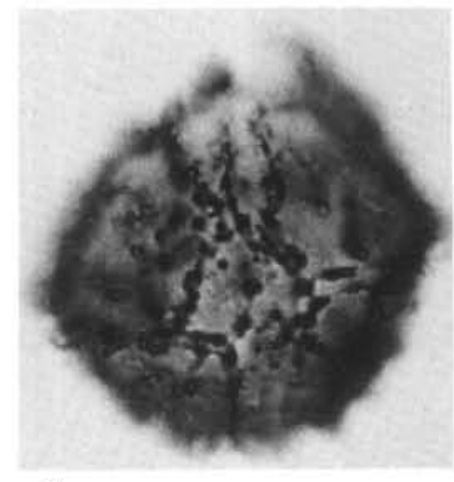

7

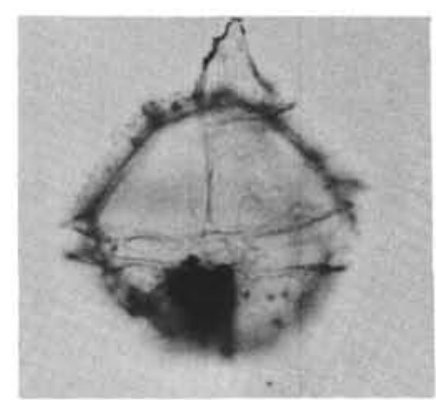

9

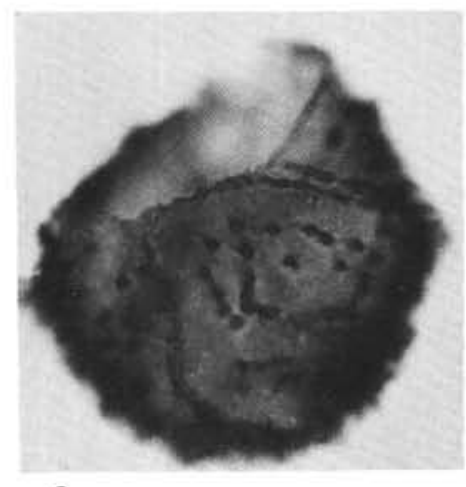

8

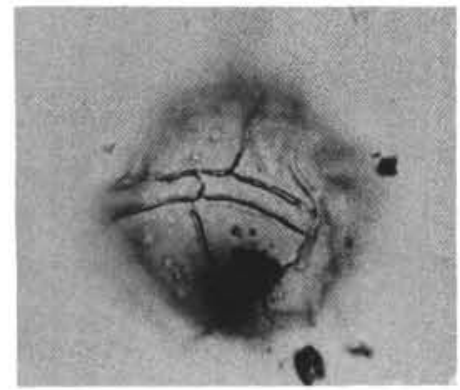

10 


\section{PLATE 8}

All magnifications $\times 500$ unless otherwise stated

Figure $1 \quad$ Gonyaulacysta sp. cf. G. apionis (Cookson and Eisenack). Sample 330-3-2, 103-105 cm. Slide S3157/2, 20.6:131.6.

Figures 2, 3 Gonyaulacysta helicoidea (Eisenack and Cookson). 2. Sample $330-3-2,103-105 \mathrm{~cm}$. Slide S3157/2, 17.2:102.5.

3. Sample 330-3-2, 103-105 cm. Slide S3157/2, 14.8:132.3.

Figure $4 \quad$ Scriniodinium dictyotum Cookson and Eisenack. Sample 330-4-2, 131-133 cm. Slide S3162/3, 13.6:127.9.

Figure $5 \quad$ Gonyaulacysta helicoidea (Eisenack and Cookson). Sample 327A-22-1, 126-129 cm. Slide S3103/1, 14.5:103.3.

Figure 6 ?Scriniodinium sp. Sample 330-5-1, 143-146 cm. Slide S3161/1, 5.9:104.7.

Figures 7,8 Apteodinium reticulatum Singh.

7. Sample 327A-22-2, 7-10 cm. Slide ST3105/2, 13.8:106.5.

8. Sample 327A-22-1, 126-129 cm. Slide S3103/1, 17.1:100.0.

Figure 9 Apteodinium sp. cf. A. granulatum Eisenack. Sample 330-3-2, 103-105 cm. Slide S3157/2, 8.8:115.1.

Figure $10 \quad$ Nannoceratopsis sp. Sample 330-4-1, 86-88 cm. Slide S3163/3, 16.9:109.3. 


\section{PLATE 8}
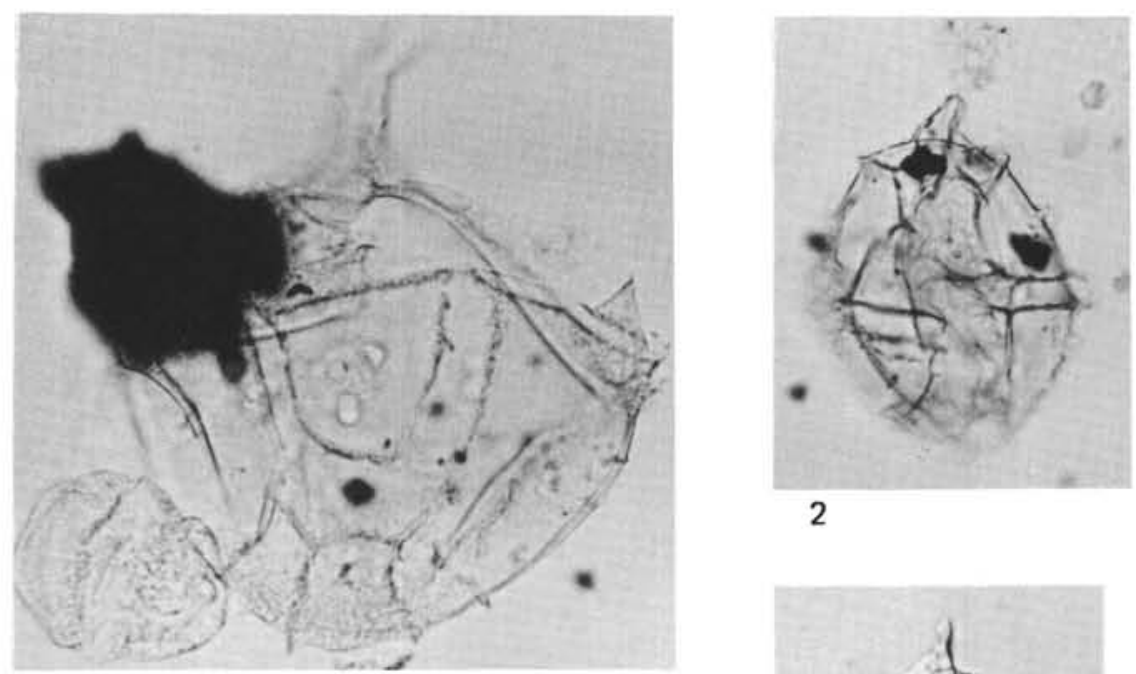

2

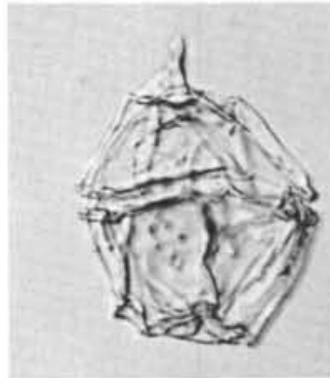

5

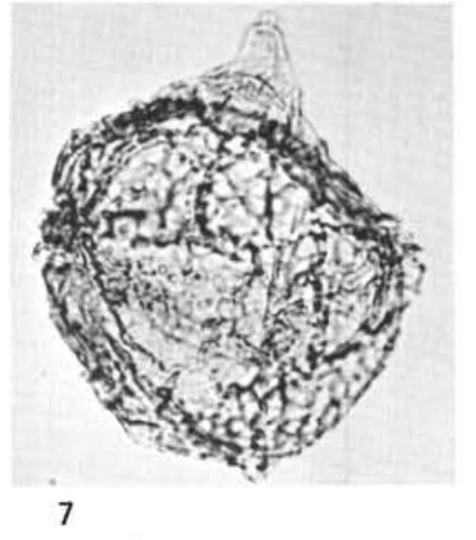

8
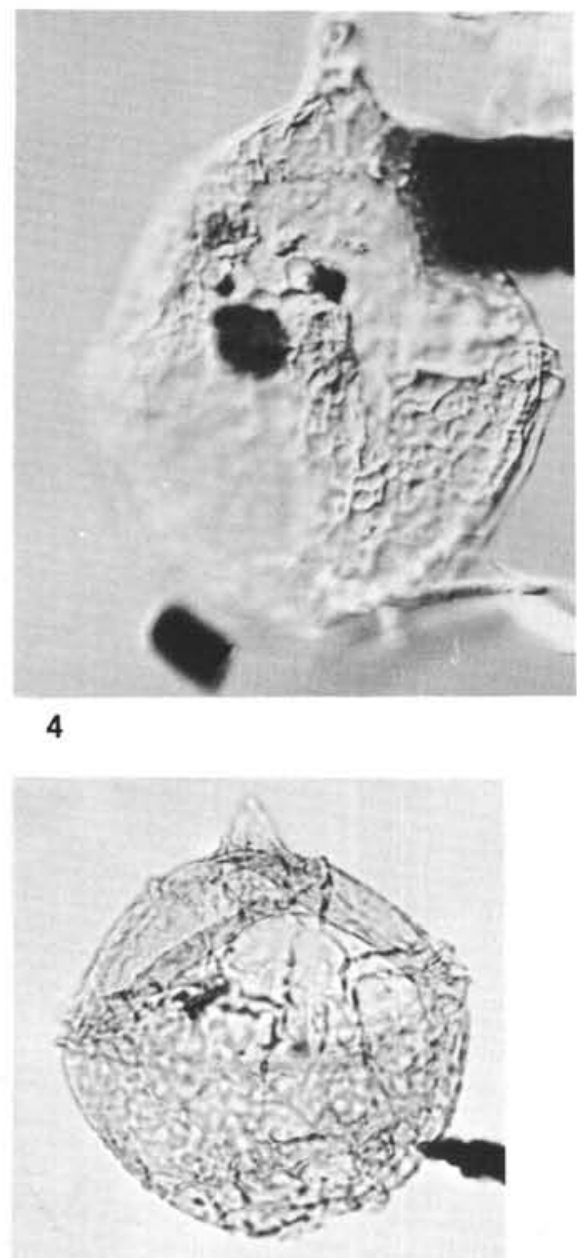

4

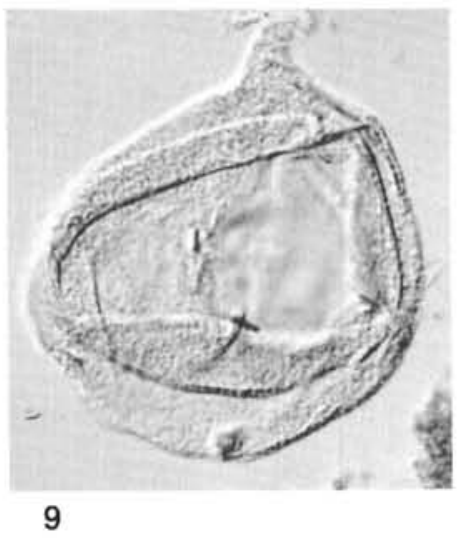

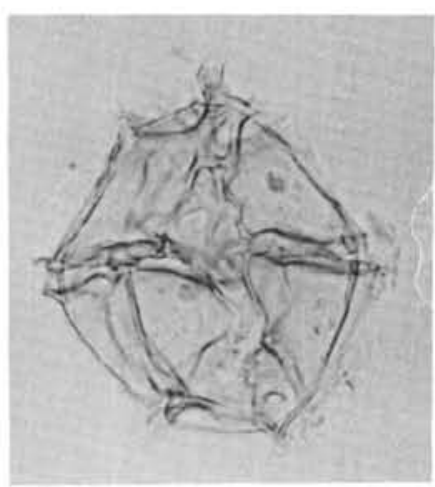

3
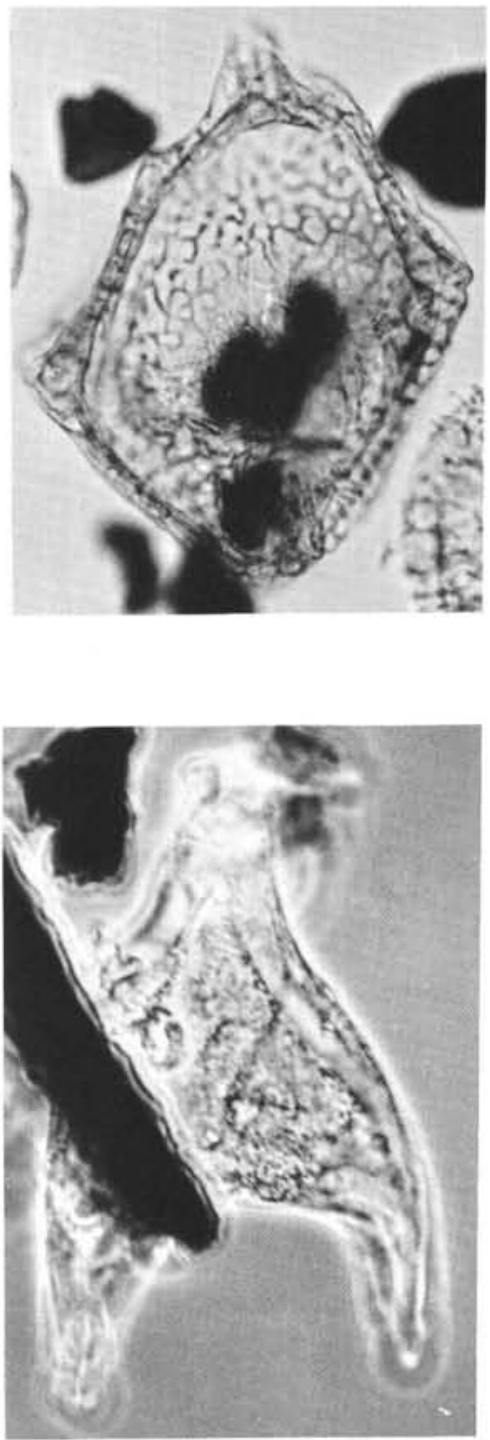

10 


\section{PLATE 9}

Gonyaulacysta spp.

All magnifications $\times 500$ unless otherwise stated.

Figure 1 Sample 330-3-2, 103-105 cm. Slide S3157/2, 15.0:103.3.

Figure 2 Sample 330-3-2, 103-105 cm. Slide S3157/2, 11.7:125.6.

Figure 3 Sample $330-3-2,103-105 \mathrm{~cm}$. Slide S3157/2, 8.7:98.6.

Figure 4 Sample 330-3-2, 103-105 cm. Slide S3157/2, 3.8:104.2, $\times 300$.

Figure 5 Sample 330-4-2, 131-133 cm. Slide S3162/3, 13.0:121.9.

Figure 6 Sample 330-4-2, 131-133 cm. Slide S3162/3, 16.5:95.7.

Figure 7 Sample 330-4-2, 131-133 cm. Slide S3162/1, 12.0:111.1.

Figure 8 Sample $330-3-2,103-105 \mathrm{~cm}$. Slide S3157/2, 9.2:124.2.

Figure 9 Sample 330-3-2, 103-105 cm. Slide S3157/2, 16.9:110.6. 
PLATE 9

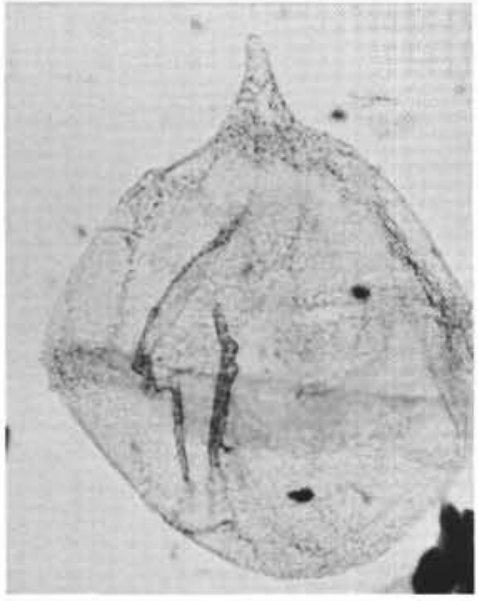

1

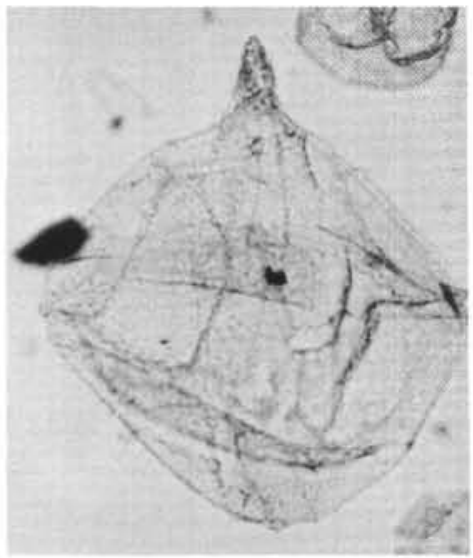

4

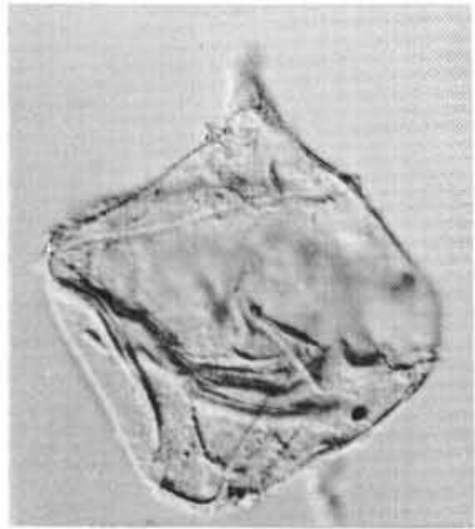

7

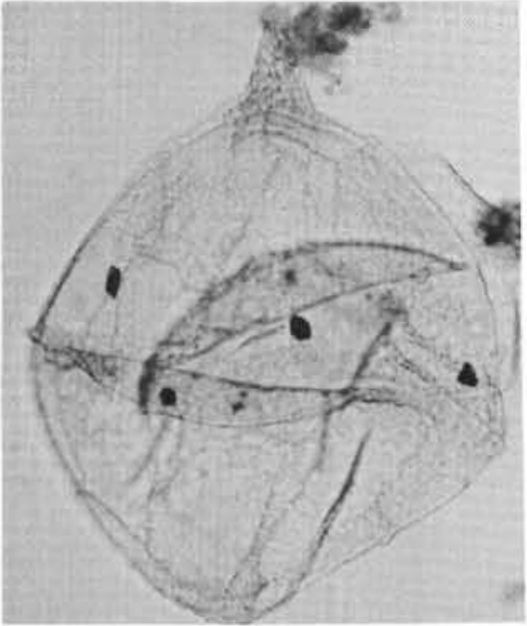

2

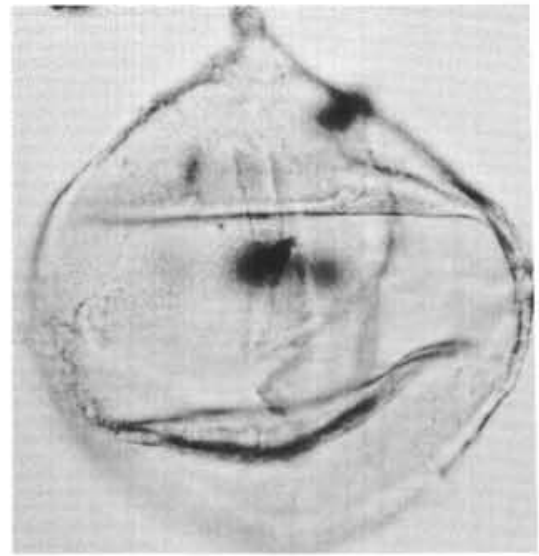

5

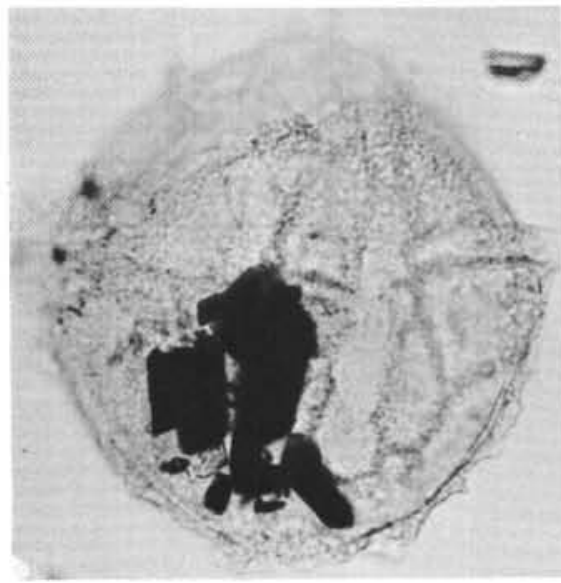

8

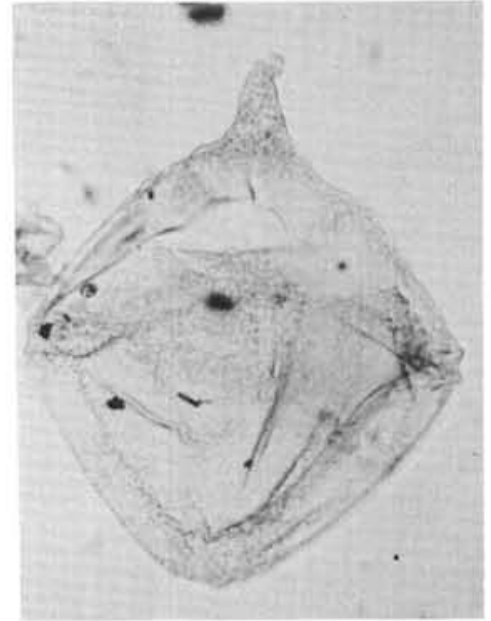

3

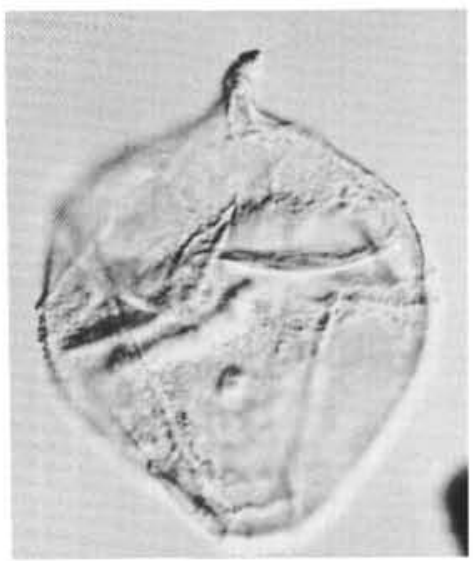

6

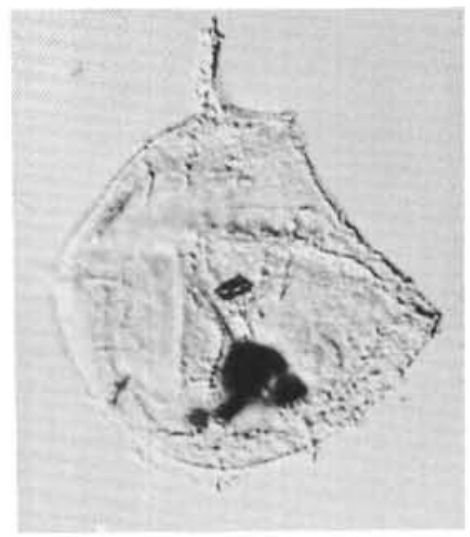

9 


\section{PLATE 10}

All magnifications $\times 500$ unless otherwise stated.

Figure 1 Apteodinium conjunctum Eisenack and Cookson. Sample 330-3-2, 103-105 cm. Slide S3157/2, 15.5:130.1.

Figure 2 ?Occisucysta sp. Sample 327A-22-1, 126-129 cm. Slide S3103/1, 17.8:119.5.

Figure 3 ?Apteodinium sp. Sample 327A-22-1, 126-129 cm. Slide S3103/1, 11.8:107.1.

Figures 4,5 ?Apteodinium sp. Sample 327 A-22-2, 7-10 cm. Slide ST3105/2, 15.4:111.8.

Figure 6 ?Apteodinium sp. Sample 327A-22-2, 7-10 cm. Slide ST3105/3, 11.4:113.8.

Figure 7 ?Occisucysta sp. Sample 327A-22-1, 126-129 cm. Slide S3103/1, 18.1:119.8.

Figure $8 \quad$ ?Occisucysta $\mathrm{sp}$. Sample 330-5-1, 143-146 cm. Slide S3161/1, 9.3:107.9.

Figure 9 ?Occisucysta $\mathrm{sp}$. Sample 330-5-1, 143-146 cm. Slide S3161/1, 118.7:5.9.

Figure $10 \quad$ Apteodinium $\mathrm{sp}$. Sample 330-3-2, 103-105 cm. Slide S3157/2, 10.8:106.7.

Figure 11 Apteodinium sp. Sample 327A-22-1, 126-129 cm. Slide S3103/1, 6.6:99.9.

Figures 12,13 Gonyaulacysta sp. Sample 330-3-2, 103-105 cm. Slide S3157/2, 6.9:112.8. 
PLATE 10

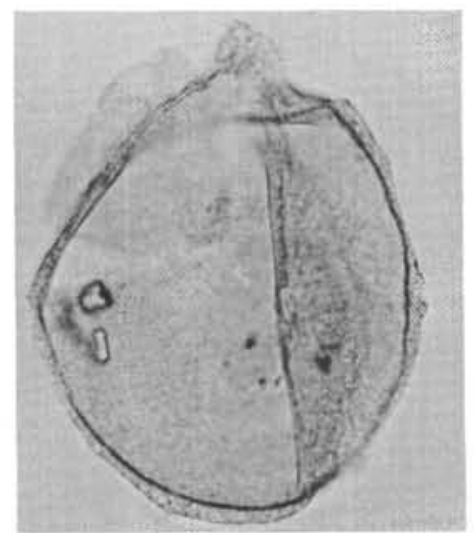

1

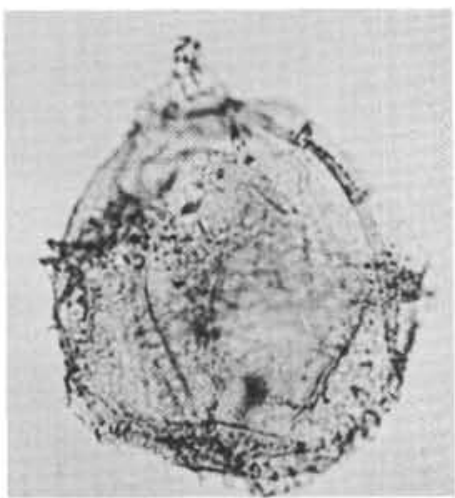

4

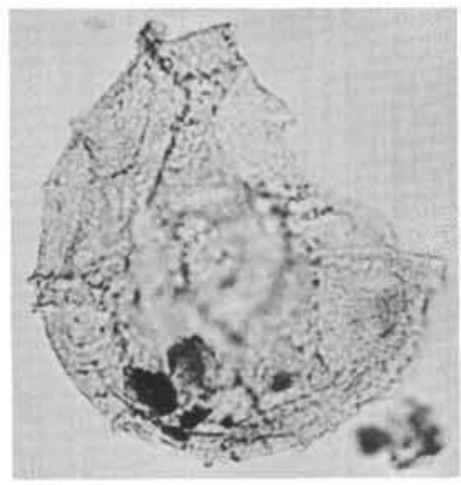

7

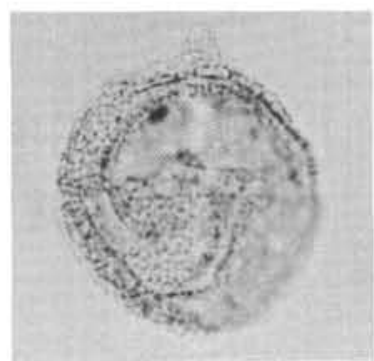

10

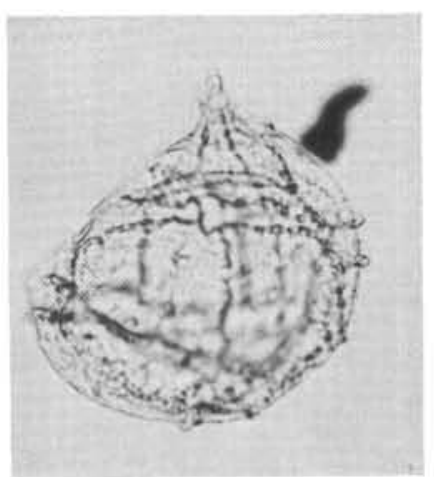

2

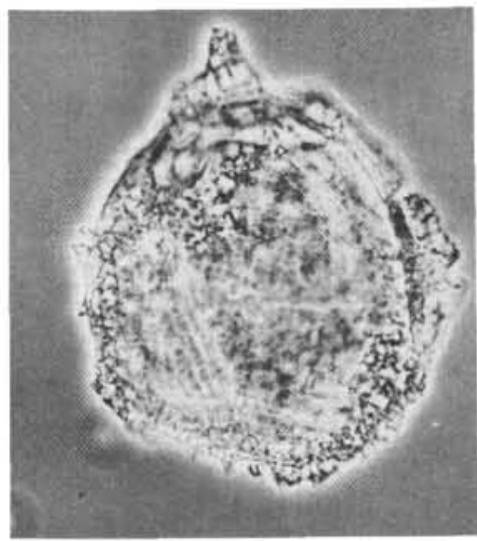

5

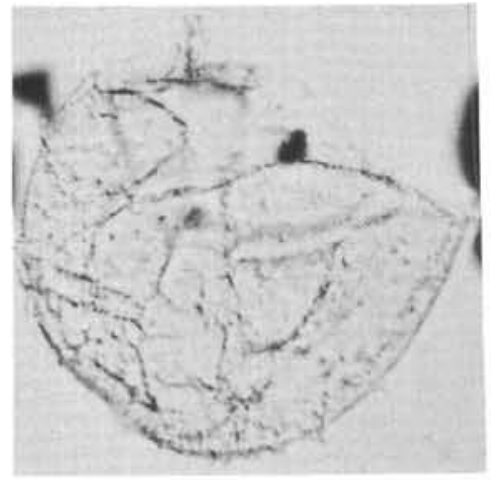

8

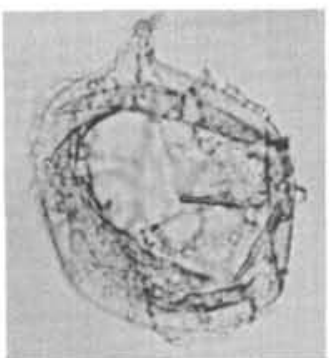

11

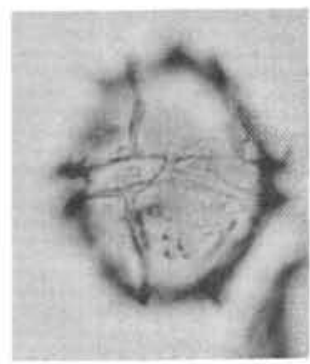

12

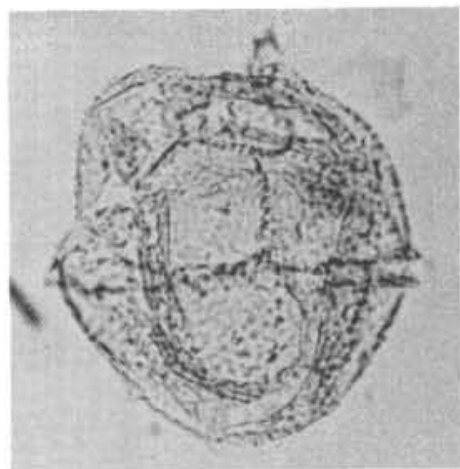

3

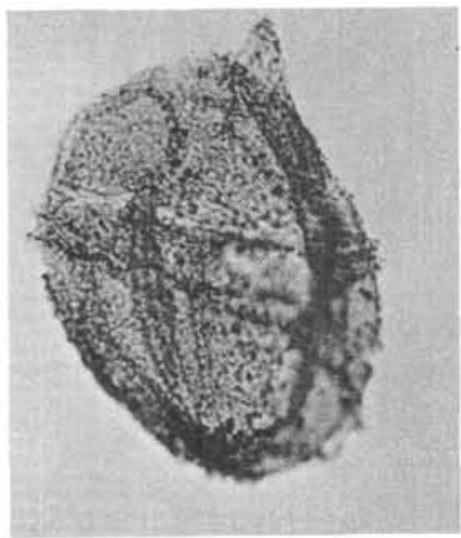

6

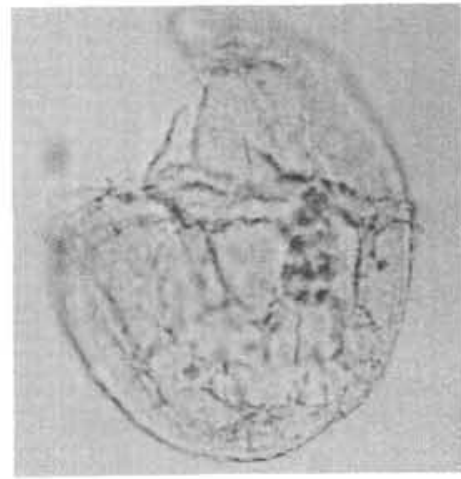

9 


\section{PLATE 11}

All magnifications $\times 500$ unless otherwise stated

Figures 1,2 Gonyaulacysta sp. Sample 330-3-2, 103-105 cm. Slide S3157/2, 7.4:99.8.

Figures 3, 4 Gonyaulacysta helicoidea (Eisenack and Cookson). Sample 327A-26-2, 126-130 cm. Slide S3118/1, 5.6:108.7.

Figure 5 Sp. indet. Sample 330-12-6, 120-122 cm. Slide S3130/1, 14.5:130.2.

Figure 6 Leptodinium sp. Sample 330-12-6, 120-122 cm. Slide S3130/1, 8.9:127.8.

Figures 7, 8 Sp. indet. Sample 330-4-2, 131-133 cm. Slide S3162/1, 121.4:15.7.

Figure $9 \quad$ Psaligonyaulax apatelum (Cookson and Eisenack). Sample 330-4-2, 131-133 cm. Slide S3162/3, 5.5:99.5.

Figure $10 \quad$ Spiniferites $\mathrm{sp}$. cf. S. cingulatus (O. Wetzel). Sample 328-12-1, 69-71 cm. Slide ST3192/1, 9.0:103.7.

Figures 11, 12 Spiniferites cingulatus granulatus (Clarke and Verdier). Sample 328-12, CC. Slide ST3195/7, 14.6:107.7.

Figure 13 Spiniferites cingulatus (O. Wetzel). Sample 327A22-1, 126-129 cm. Slide S3103/1, 2.6:115.2.

Figure 14 Spiniferites ramosus (Ehrenberg). Sample 327A22-2, 7-10 cm. Slide ST3105/19, 16.7:108.2.

Figures 15, 16 Spiniferites cingulatus (O. Wetzel). Sample 327A12-1, 109-112 cm. Slide ST3096/2, 17.4:103.1.

Figure 17 Cleistosphaeridium sp. Sample 327A-22-1, 126-129 $\mathrm{cm}$. Slide S3103/1, 6.2:121.4.

Figure 18 Spiniferites ramosus (Ehrenberg). Sample 327A22-1, 126-129 cm. Slide S3103/1, 15.7:96.8.

Figures 19,20 Spiniferites cingulatus (O. Wetzel).

19. Sample 327A-9, CC. Slide ST3093/2, 104.6:14.1.

20. Sample 327A-8, CC. Slide ST3092/4, 12.7:108.1.

Figure 21 Hystrichosphaeridium sp. Sample 327A-9, CC. Slide ST3093/9, 12.2:108.2.

Figure 22 ?Pareodinia sp. Sample 330-12-6, 120-122 cm. Slide S3130/1, 19.0:99.1. 
PLATE 11
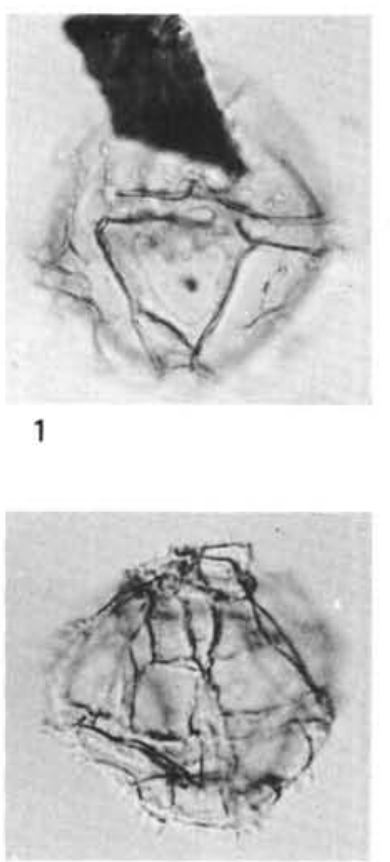

5
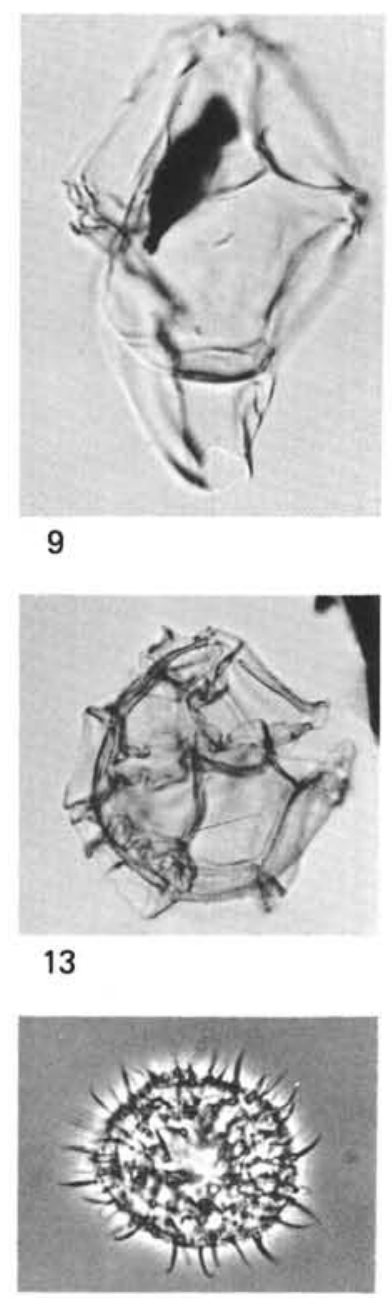

17

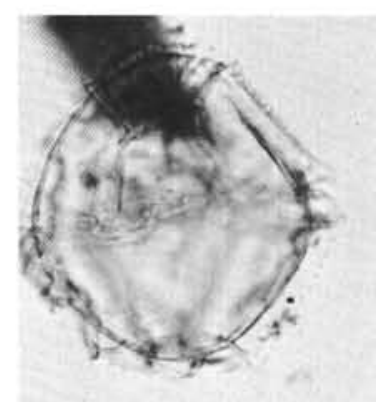

2
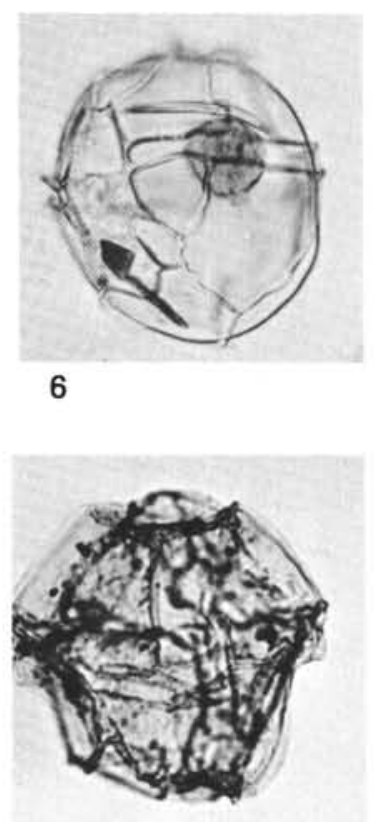

10

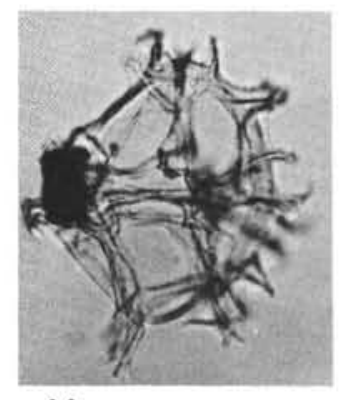

14

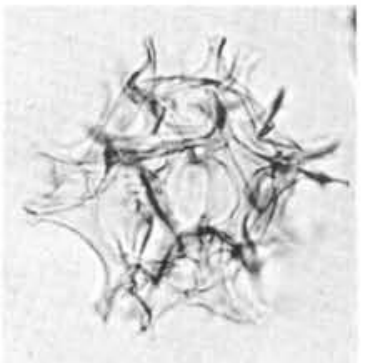

18

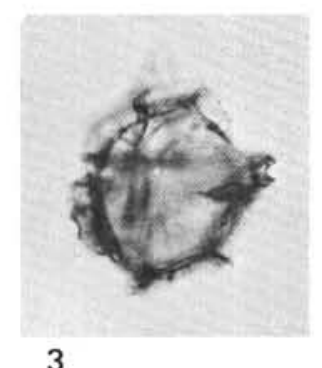

3

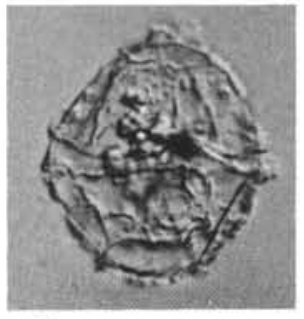

7

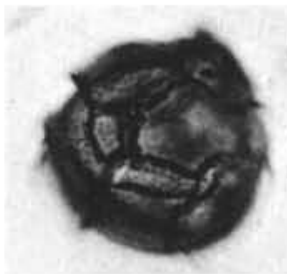

11

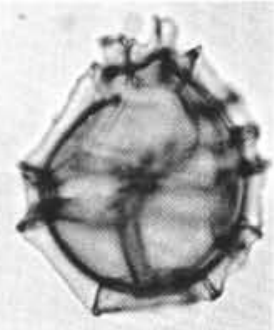

15

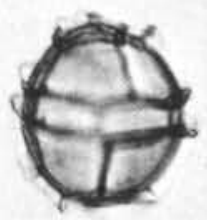

19

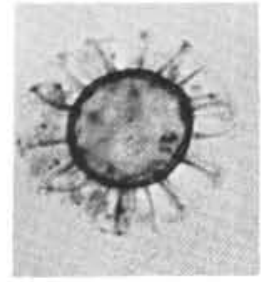

21
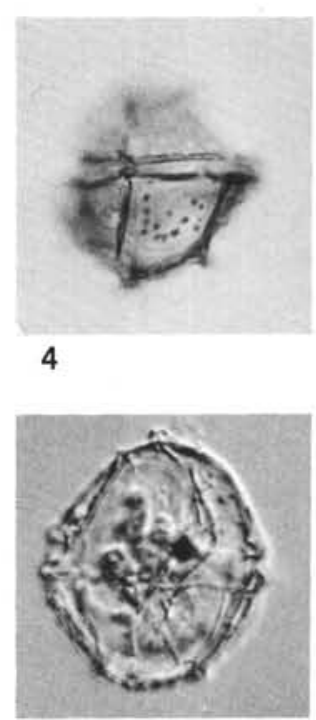

8

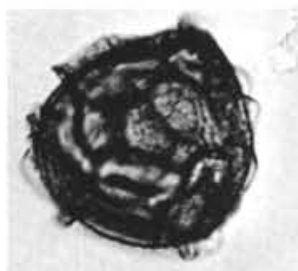

12

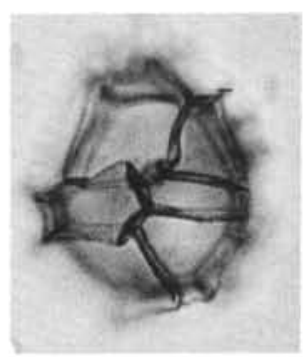

16

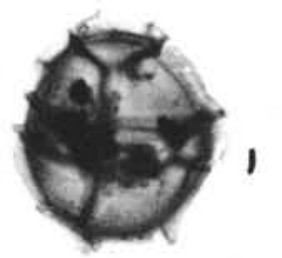

20

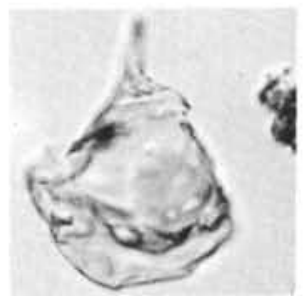

22 


\section{PLATE 12}

All magnifications $\times 500$ unless otherwise stated

Figures 1,2 Gen. et sp. indet.

1. Sample $328-11-1,42-44 \mathrm{~cm}$. Slide ST3182/8, 13.3:110.2.

2. Sample $328-11-1,42-44 \mathrm{~cm}$. Slide ST3182/9, 15.1:110.0.

Figure 3 Cometodinium sp. Sample 327A-22-2, 7-10 cm. Slide ST3105/22, 15.6:105.1.

Figure 4 Cleistosphaeridium sp. Sample 328B-7-3, 104-106 $\mathrm{cm}$. Slide ST3199/6, 15.5:108.6.

Figure $5 \quad$ Hystrichodinium sp. Sample 328B-7-3, 104-106 cm. Slide ST3199/2, 11.7:105.3.

Figure 6 Conosphaeridium striatoconus (Deflandre and Cookson). Sample 328B-7-6, 4-7 cm. Slide ST3202/1, 16.1:105.5.

Figure $7 \quad$ Cleistosphaeridium sp. Sample 328-12, CC. Slide ST3195/4, 11.8:106.5.

Figure $8 \quad$ Sp. indet. Sample 328-12, CC. Slide ST3195/5, 12.1:108.0.

Figure 9 Sp. indet. Sample 328B-7-6, 4-7 cm. Slide ST3202/4, 12.8:106.8.

Figure $10 \quad$ Exochosphaeridium $\mathrm{sp}$. cf. E. striolatum truncatum (Davey). Sample 327A-22-1, 126-129 cm. Slide S3103/1, 14.0:97.1.

Figure 11 Exochosphaeridium sp. Sample 327A-22-1, 126-129 $\mathrm{cm}$. Slide S3103/1, 8.9:103.4.

Figures 12, 13 Hystrichosphaeridium cooksonii Singh.

12. Sample 327A-22-1, 126-129 cm. Slide $\mathrm{S} 3103 / 1,3.3: 120.1$.

13. Sample 327 A-22-2, 7-10 cm. Slide ST $3105 / 10$, $8.7: 102.2$.

Figure 14 Hystrichosphaeridium sp. Sample 328B-7-6, 4-7 $\mathrm{cm}$. Slide ST3202/7, 15.7:102.4.

Figure 15 Sp. indet. Sample 327A-22-1, 126-129 cm. Slide S3103/1, 11.5:103.7.

Figure 16 Hystrichosphaeridium sp. Sample 327A-22-1, 126$129 \mathrm{~cm}$. Slide S3103/1, 2.9:109.3.

Figure 17 Sp. indet. Sample 328B-7-3, 104-106 cm. Slide ST3199/5, 13.2:107.4. 
PLATE 12

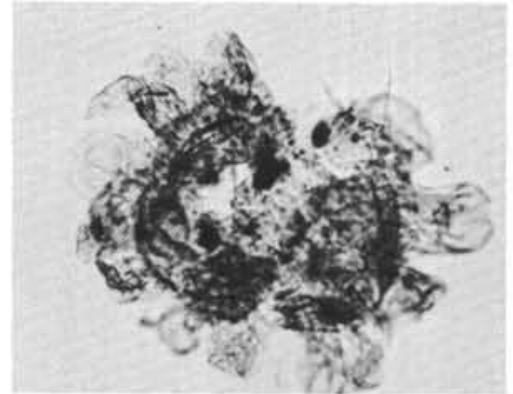

1

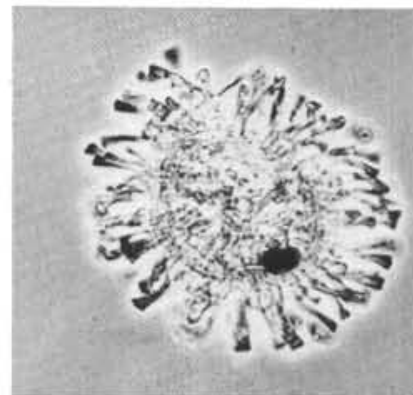

4

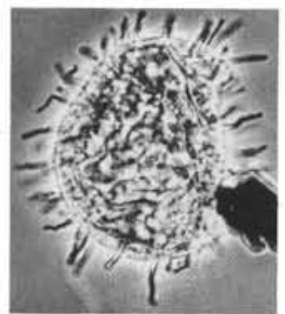

7

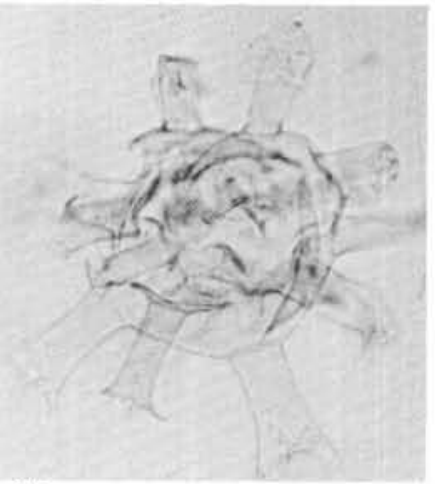

12

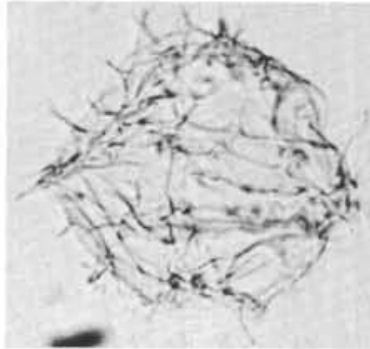

15

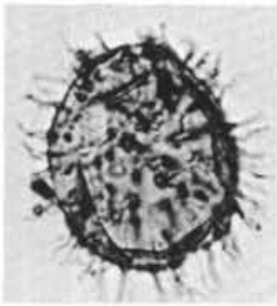

8

13

16

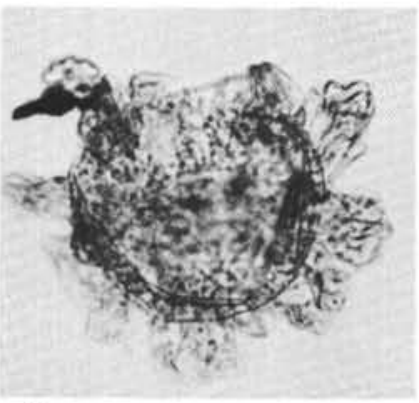

2

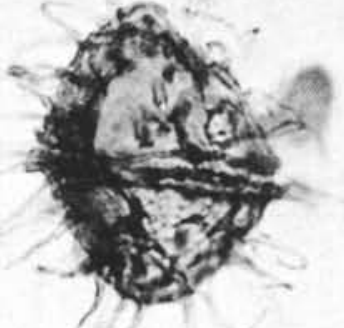

5

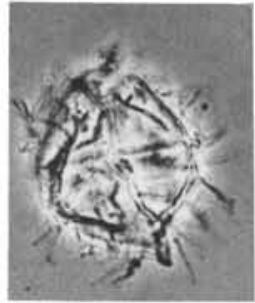

9

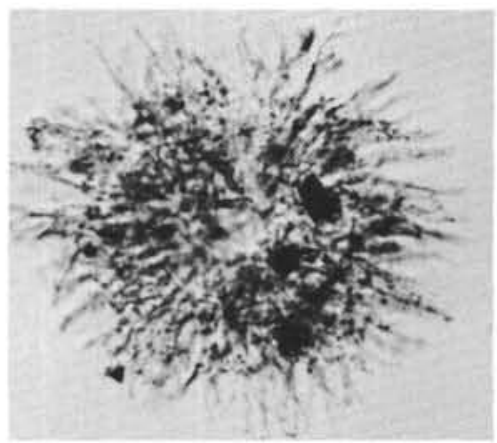

3

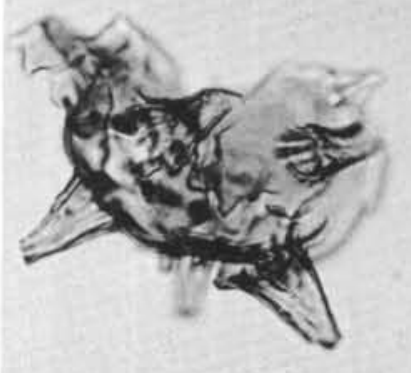

6
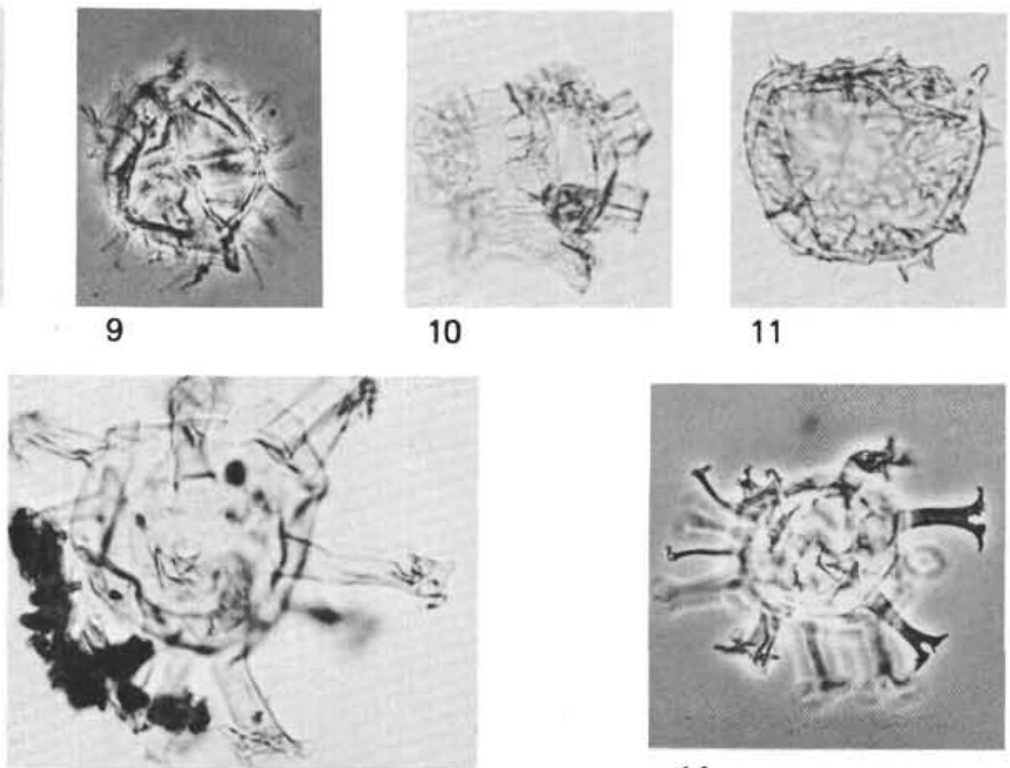

11

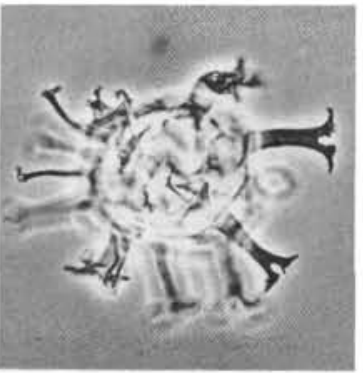

14

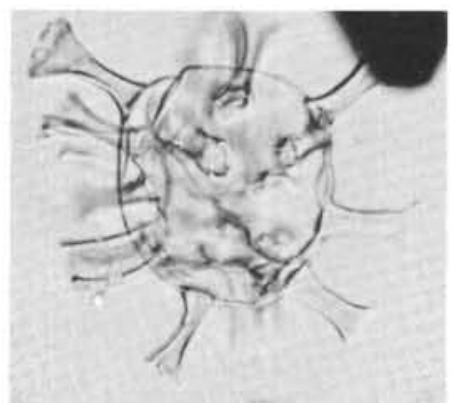

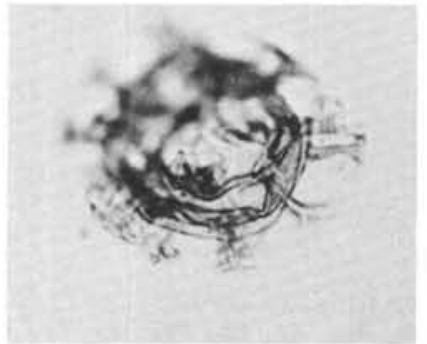

17 


\section{PLATE 13}

All magnifications $\times 500$ unless otherwise stated

Figures 1-3 Oligosphaeridium complex (White).

1. Sample 330-3-2, 103-105 cm. Slide S3157/2, 15.8:122.0.

2. Sample 330-3-2, 103-105 cm. Slide S3157/2, 11.9:119.6.

3. Sample 327 A-22-1, $126-129 \mathrm{~cm}$. Slide $\mathrm{S} 3103 / 1$, 8.9:103.4.

Figure $4 \quad$ Systematophora sp. cf. S. fasciculigera Klement. Sample 330-3-2, 103-105 cm. Slide S3157/2, 3.7:125.9.

Figures 5, 6 Sp. indet. Sample 330-12-6, 120-122 cm. Slide S3130/1, 7.8:123.4.

Figure $7 \quad$ Canningia sp. Sample 330-3-2, 103-105 cm. Slide S3157/2, 7.7:98.8.

Figure 8 ?Meiourogonyaulax sp. Sample 330-3-2, 103-105 $\mathrm{cm}$. Slide S3157/2, 5.8:122.6.

Figures 9, $10 \quad$ Palaeostomocystis sp. Sample 327A-12-4, 134-139 $\mathrm{cm}$. Slide ST3099/5, 11.1:106.1. 


\section{PLATE 13}
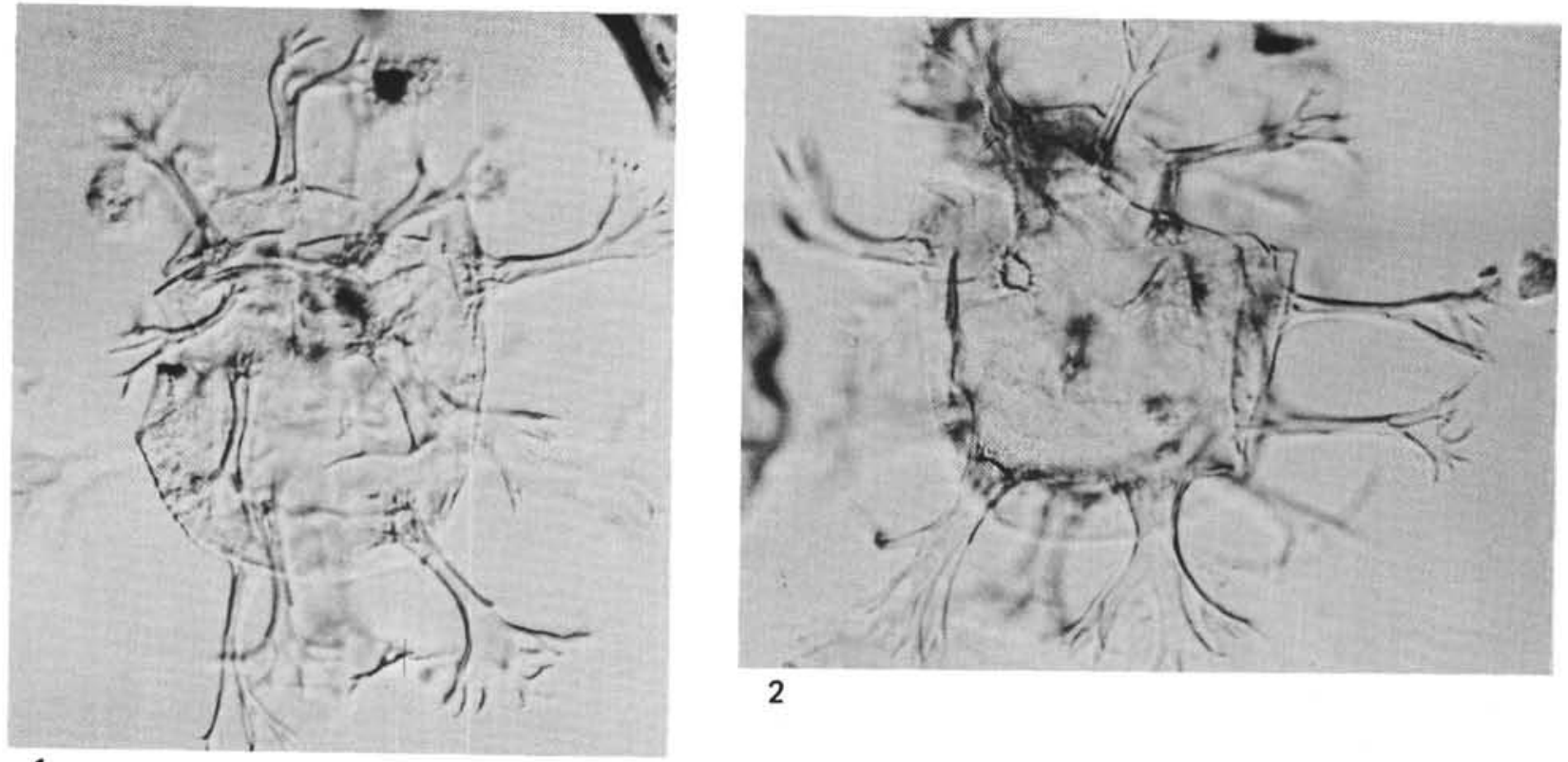

2

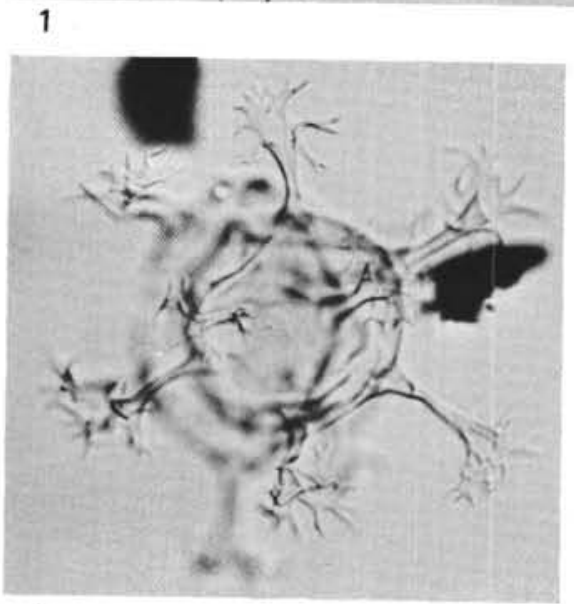

3
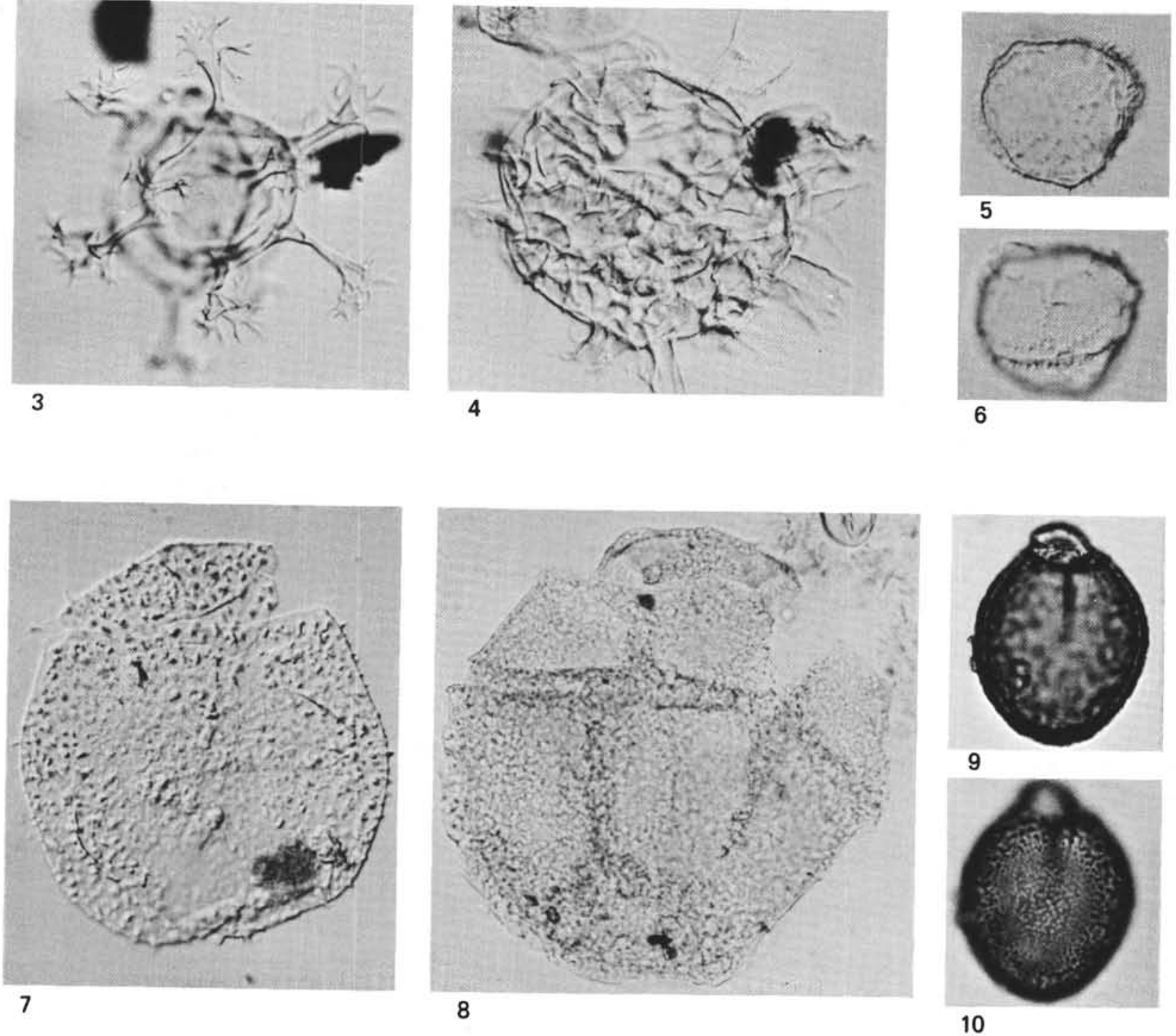


\section{PLATE 14}

All magnifications $\times 500$ unless otherwise stated

Figure 1 Tanyosphaeridium isocalamus (Deflandre and Cookson). Sample 330-3-2, 103-105 cm. Slide S3157/2, 9.5:129.1.

Figure 2 Prolixosphaeridium parvispinum (Deflandre). Sample 327A-22-1, 126-129 cm. Slide S3103/1, 4.9:107.2.

Figures 3,4 Tenua hystrix Eisenack.

3. Sample 327A-22-1, 126-129 cm. Slide S3103/1, 6.9:106.9.

4. Sample 327A-22-1, 126-129 cm. Slide S3103/1, 17.1:113.6.

Figure 5 Tenua sp. Sample $330-7-2,55-59 \mathrm{~cm}$. Slide S3159/1, 7-3:126.6.

Figures 6,7 ?Meiourogonyaulax sp. Sample 330-14-4, 148-150 cm. Slide S3127/1, 15.1:104.0.

Figures 8-10 Sp. indet.

8, 9. Sample 330-11-4, $42-46 \mathrm{~cm}$. Slide S3136/1, 13.8:115.9.

10. Sample 330-12-6, 120-122 cm. Slide S3130/1, 12.8:117.3.

Figure 11 Sp. indet. Sample 330-4-2, 131-133 cm. Slide S3162/1, 100.9:19.5.

Figure 12 Sp. indet. Sample 330-3-2, 103-105 cm. Slide S3157/2, 5.1:119.1.

Figure 13 Sp. indet. Sample 327A-22-1, 126-129 cm. Slide S3103/1, 7.2:114.0.

Figure 14 Chytroeisphaeridia sp. cf. C. chyrtroeides Sarjeant. Sample 330-4-2, 131-133 cm. Slide S3162/3, 7.3:96.4.

Figure 15 Gen. et sp. indet. Sample 327A-12-4, 134-139 cm. Slide ST3099/1, 9.7:105.9.

Figures 16, 17 Chytroeisphaeridia sp. Sample 330-4-2, 131-133 $\mathrm{cm}$. Slide S3162/3, 7.5:103.2.

Figure 18 Gen. et. sp. indet. Sample 330-3-2, 103-105 cm. Slide S3157/2, 9.9:125.3. 
PLATE 14

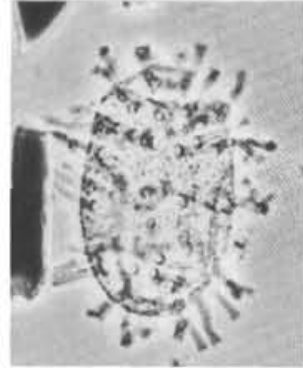

1

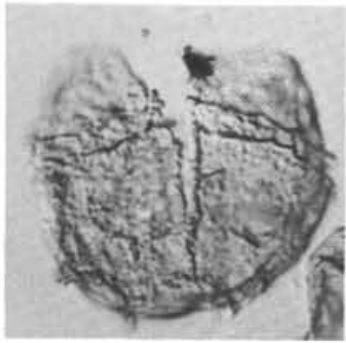

6

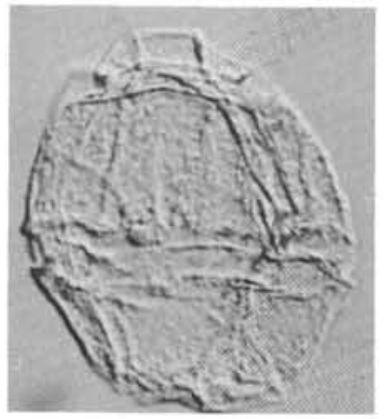

11

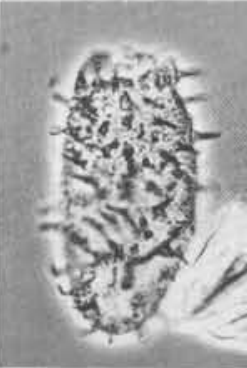

2

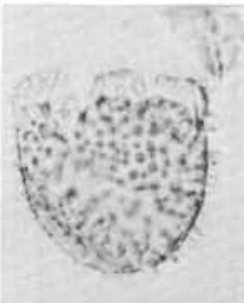

3

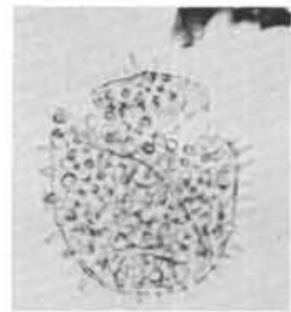

4

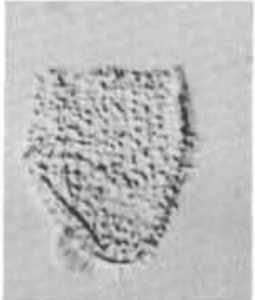

5

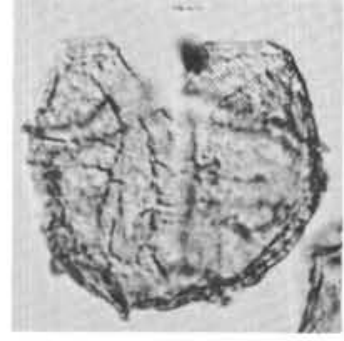

7

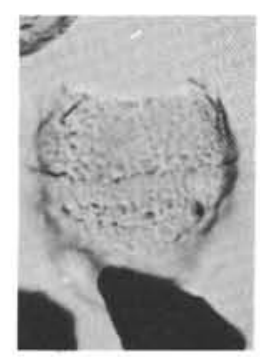

8

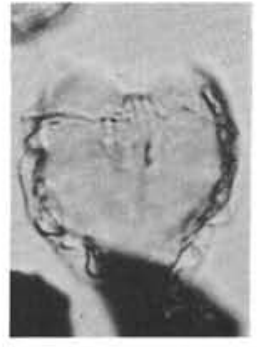

9

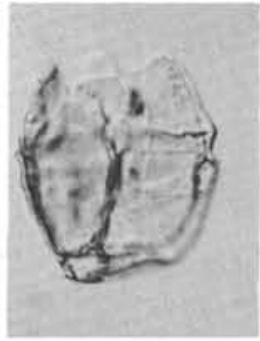

10

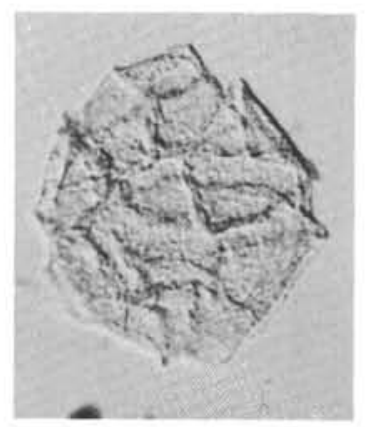

12

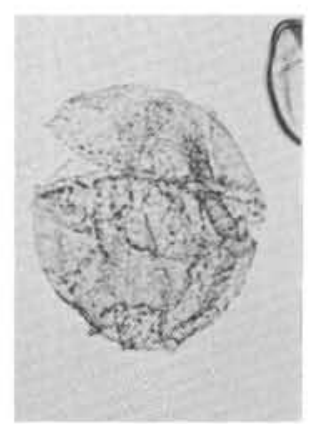

13

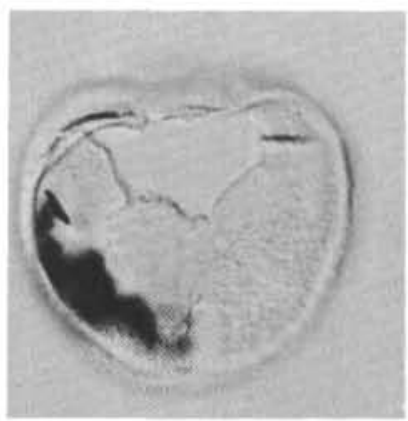

14

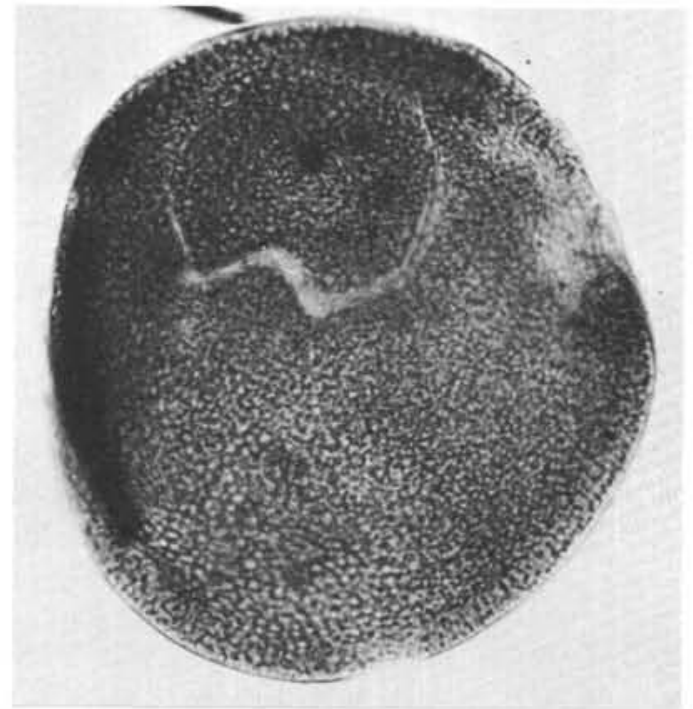

15

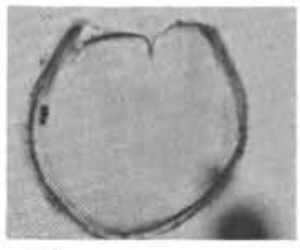

17

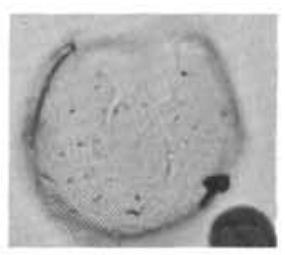

16

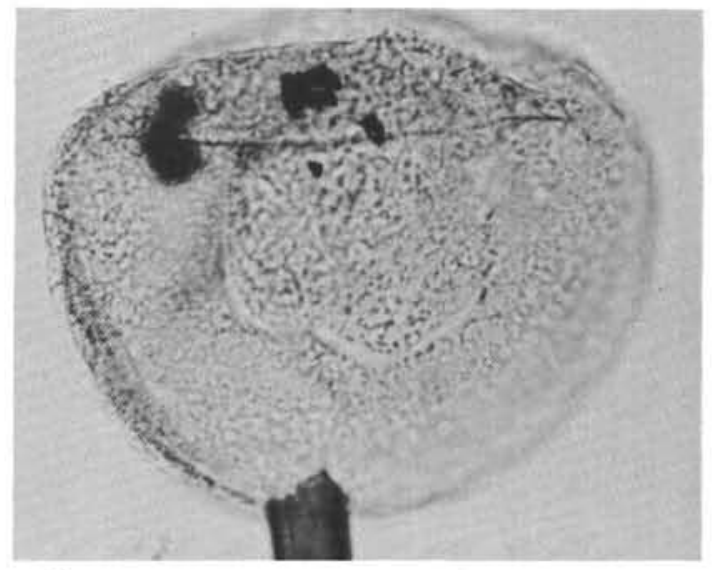

18 


\section{PLATE 15}

All magnifications $\times 500$ unless otherwise stated

Figures 1,2 Gen. et sp. indet.

1. Sample 330-3-2, 103-105 cm. Slide S3157/2, 19.8:99.1.

2. Sample 330-3-2, 103-105 cm. Slide S3157/2, 16.7:128.7.

Figure 3 Gen. et sp. indet. Sample 330-3-2, 103-105 cm. Slide S3157/2, 18.2:100.1.

Figure 4 Gen. et sp. indet. Sample 330-3-2, 103-105 cm. Slide S3157/2, 7.0:124.7.

Figure $5 \quad$ Palaeostomocystis $\mathrm{sp}$. Sample 328-11-1, 42-44 cm. Slide ST3182/2, 15.2:109.7.

Figure 6 Gen. et sp. indet. Sample 330-3-2, 103-105 cm. Slide S3157/2, 20.0:106.5.

Figure 7 Palaeostomocystis sp. Sample 328B-7-6, 4-7 cm. Slide ST3202/6, 13.8:105.7.

Figure 8 Meiourogonyaulax sp. Sample 330-3-2, 103-105 $\mathrm{cm}$. Slide S3157/2, 3.7:126.9.

Figure 9 Gen. et sp. indet. Sample 327A-22-1, 126-129 cm. Slide S3103/1, 20.2:104.9.

Figure $10 \quad \mathrm{Sp}$. indet. Sample 327A-22-2, 7-10 cm. Slide S3105/7, 12.3:108.9.

Figure $11 \quad \mathrm{Sp}$. indet. Sample $330-7-2,55-59 \mathrm{~cm}$. Slide S3159/1, 4.6:116.7. 
PLATE 15
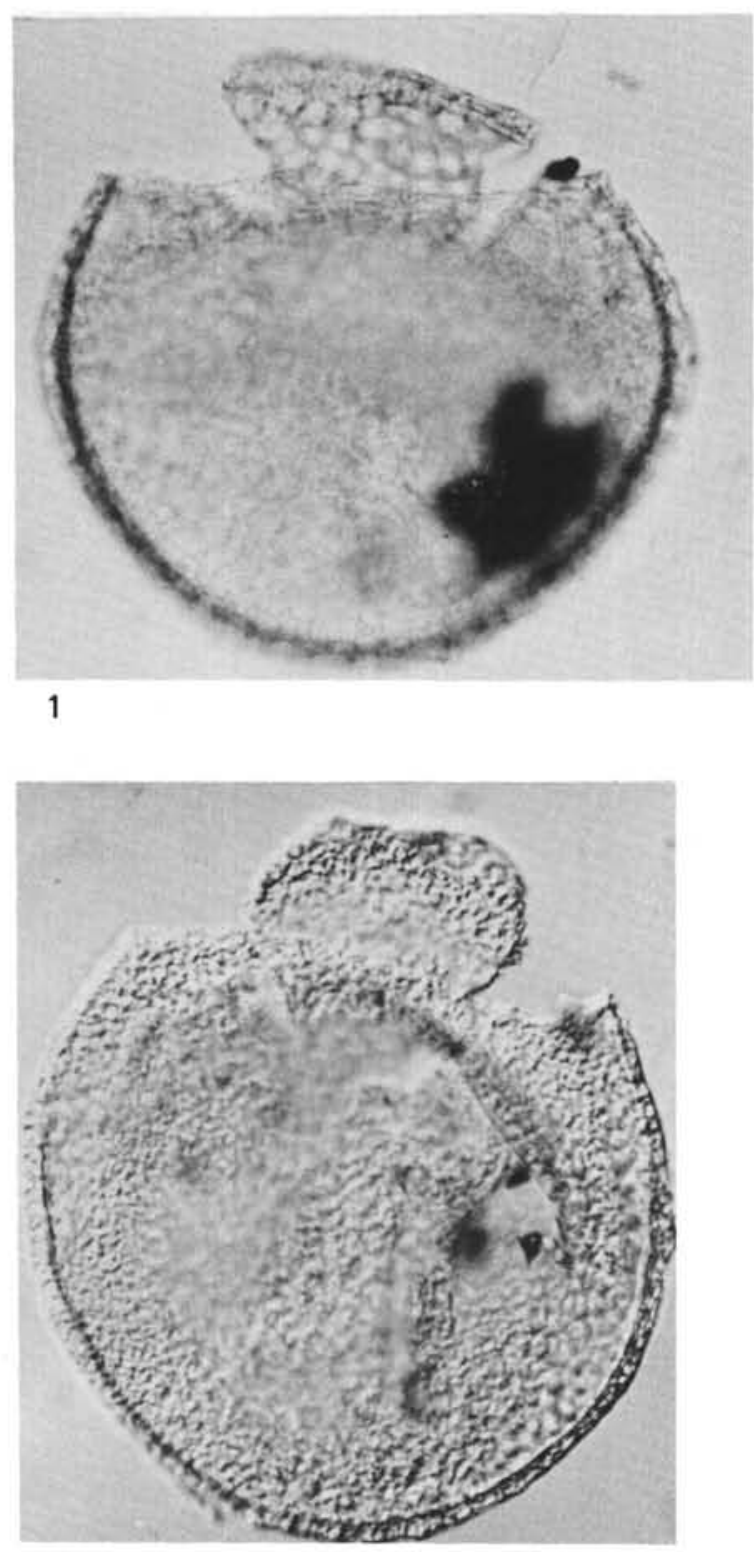

3

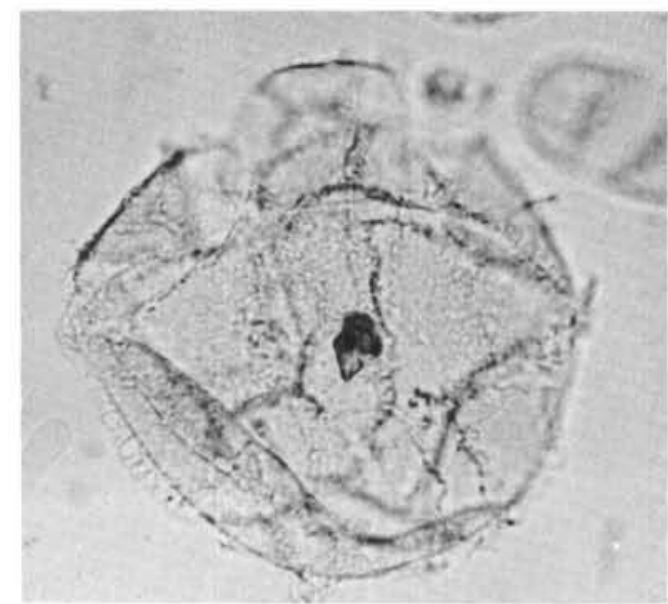

8

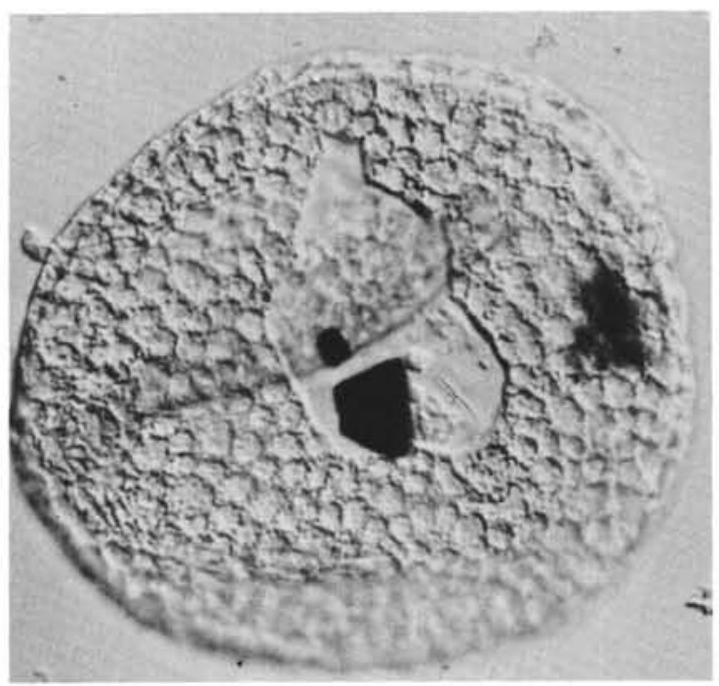

2
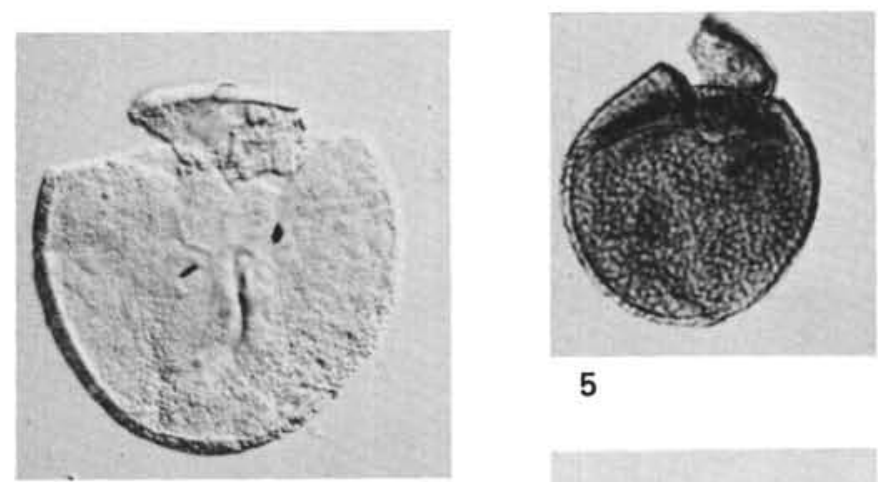

5

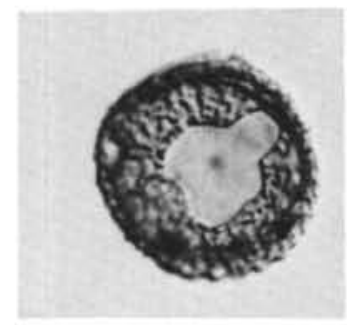

7

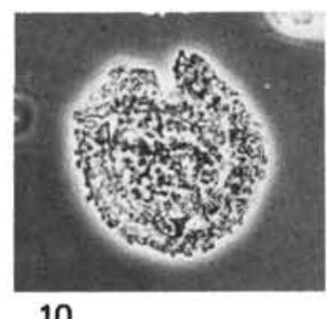

10

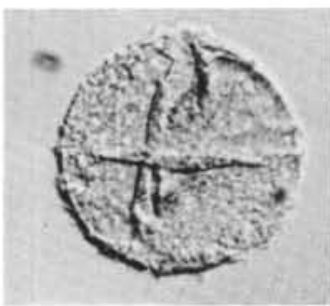

11 


\section{PLATE 16}

All magnifications $\times 500$ unless otherwise stated

Figures 1, 2 Meiourogonyaulax sp.

1. Sample 330-4-1, 86-88 cm. Slide S3163/1, 10.0:114.1.

2. Sample $330-4-1,86-88 \mathrm{~cm}$. Slide S3163/1, $6.0: 105.5$.

Figure 3 Cyclonephelium sp.cf. C. distinctum Deflandre and Cookson. Sample 330-3-2, 103-105 cm. Slide S3157/2, 7.5:99.2.

Figure 4 Canningia sp.

Sample 330-3-2, 103-105 cm. Slide S3157/2, 3.7:111.1

Figure 5 Gen. et sp. indet. Sample 330-5-1, 143-146 cm. Slide S3161/1, 6.7:116.8.

Figure 6 Gen. et sp. indet. Sample 327A-22-2, 126-129 cm. Slide ST3105/26, 13.3:111.8.

Figures 7,8 Canningia $\mathrm{sp.}$

7. Sample 330-3-2, 103-105 cm. Slide S3157/2, 11.8:98.5.

8. Sample 330-3-2, 103-105 cm. Slide S3157/2, 8.4:98.2. 
PLATE 16
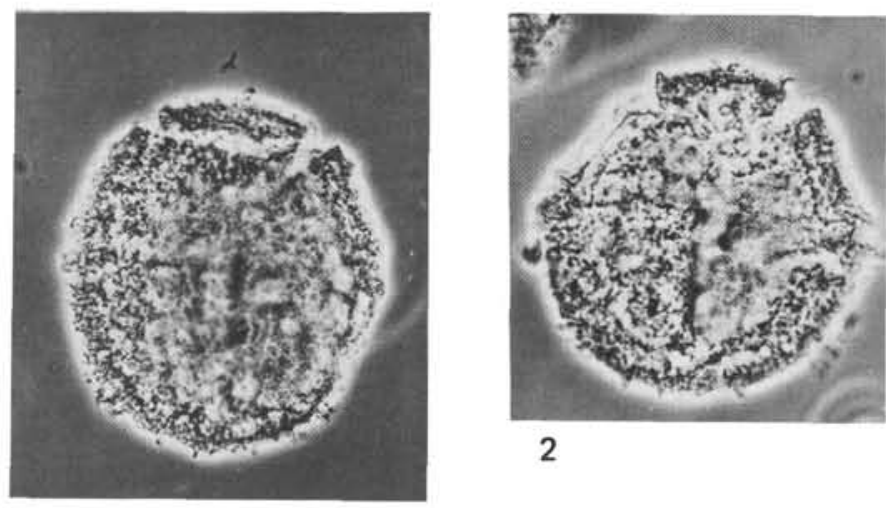

2

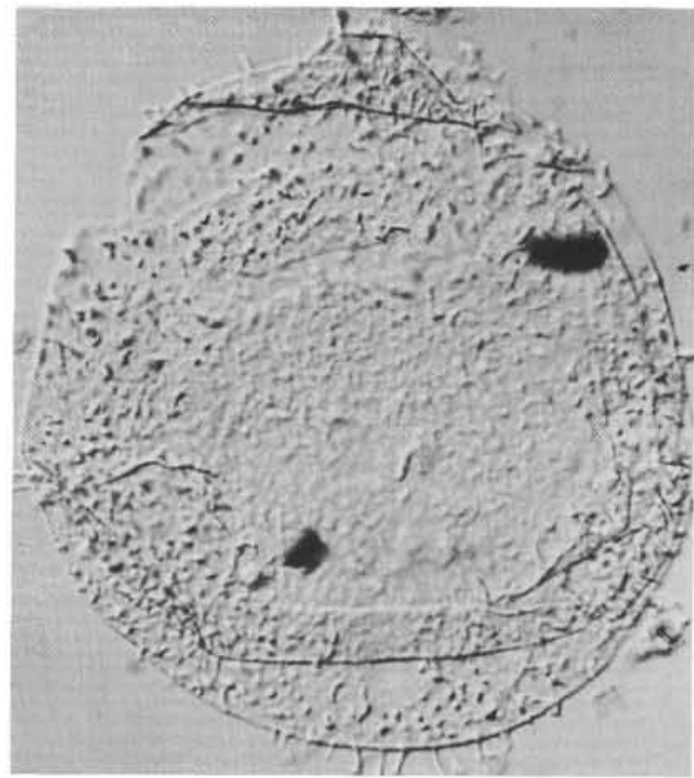

3

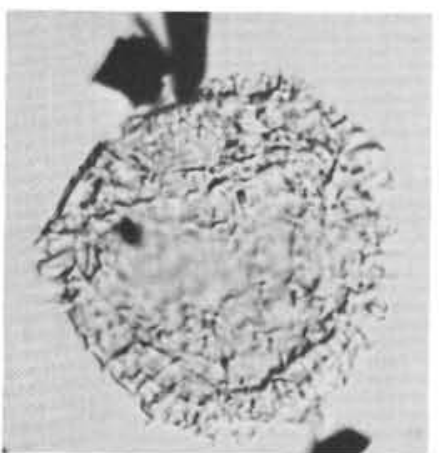

5

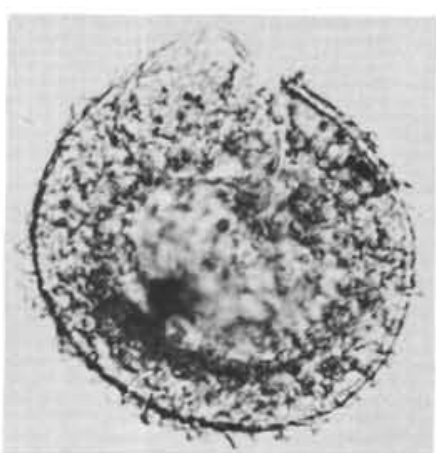

6

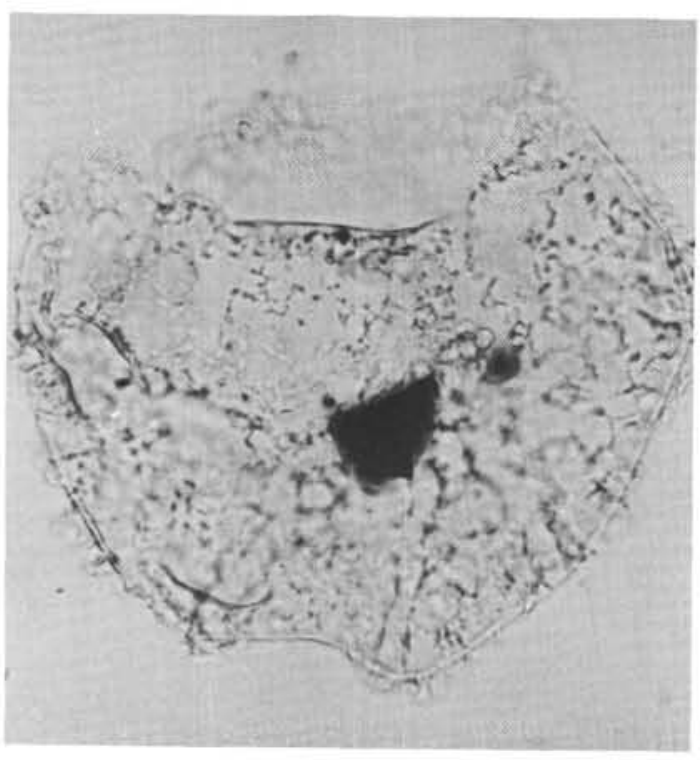

7

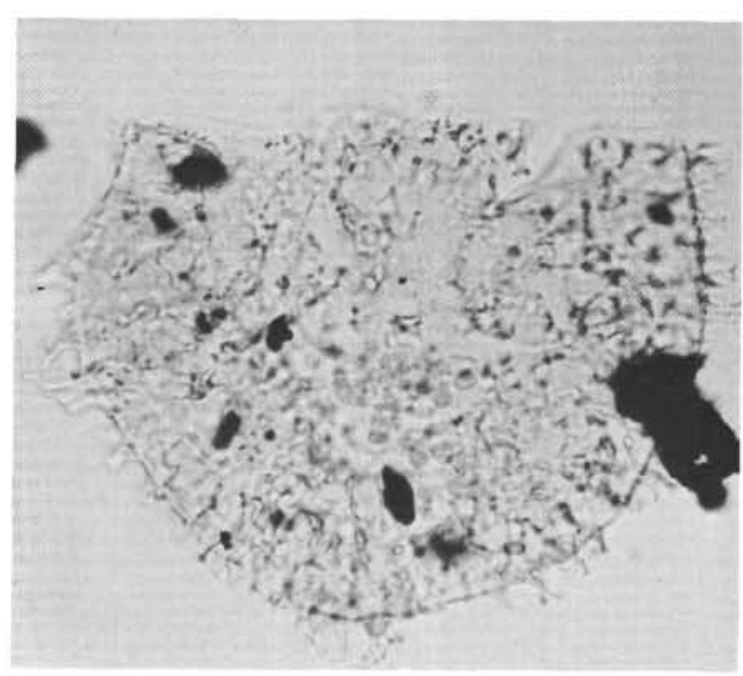

8 


\section{PLATE 17}

All magnifications $\times 500$ unless otherwise stated

Figures 1,2 Meiourogonyaulax sp. aff. M. stoveri Millioud. Sample 330-3-2, 103-105 cm. Slide S3157/2, 15.4:108.8.

Figure 3 Canningia sp. Sample 327A-22-2, 7-10 cm. Slide ST3105/19, 16.7:108.2.

Figure 4 Meiourogonyaulax sp. Sample 327A-22-1, 126-129 $\mathrm{cm}$. Slide S3103/1, 9.8:113.3.

Figure 5 ?Fromea amphora Cookson and Eisenack. Sample $330-11-5,126-128 \mathrm{~cm}$. Slide S3135/1, 4.7:102.6.

Figures 6,9 cf. Valensiella $\mathrm{sp}$. Sample 330-12-6, 120-122 cm. Slide S3130/1, 8.7:119.8.

Figure 7 Meiourogonyaulax sp. Sample 327A-22-2, 7-10 cm. Slide ST3105/17, 13.0:107.2.

Figure $8 \quad$ Canningia sp. Sample 330-3-2, 103-105 cm. Slide S3157/2, 7.1:112.2. 
PLATE 17

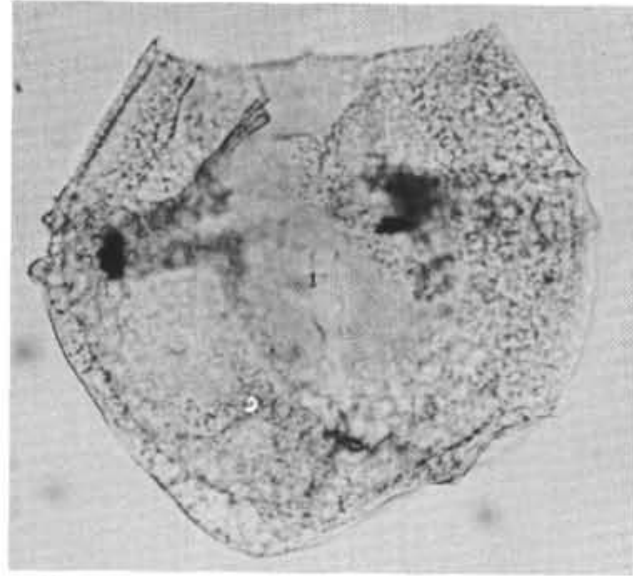

1

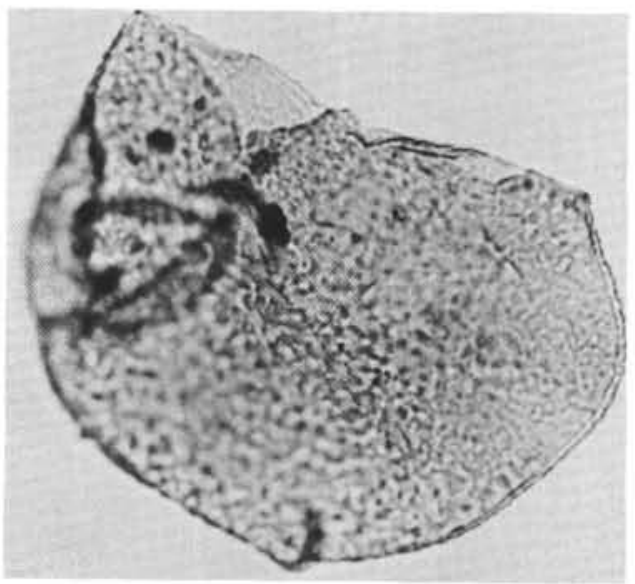

3

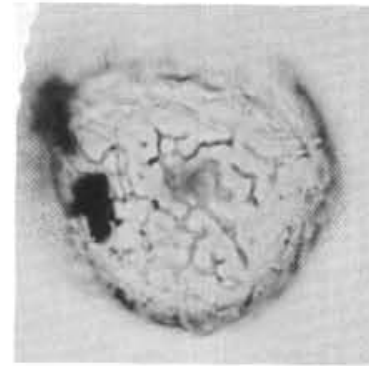

6

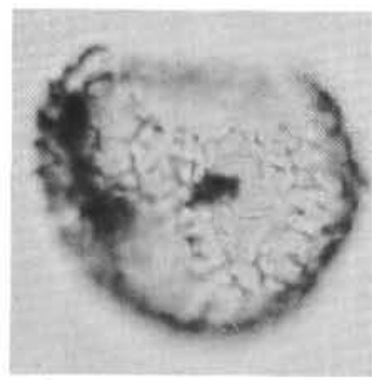

9

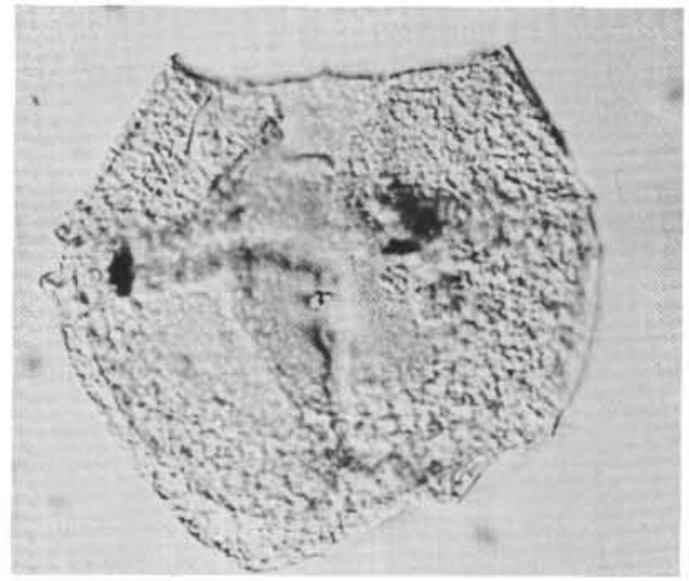

2
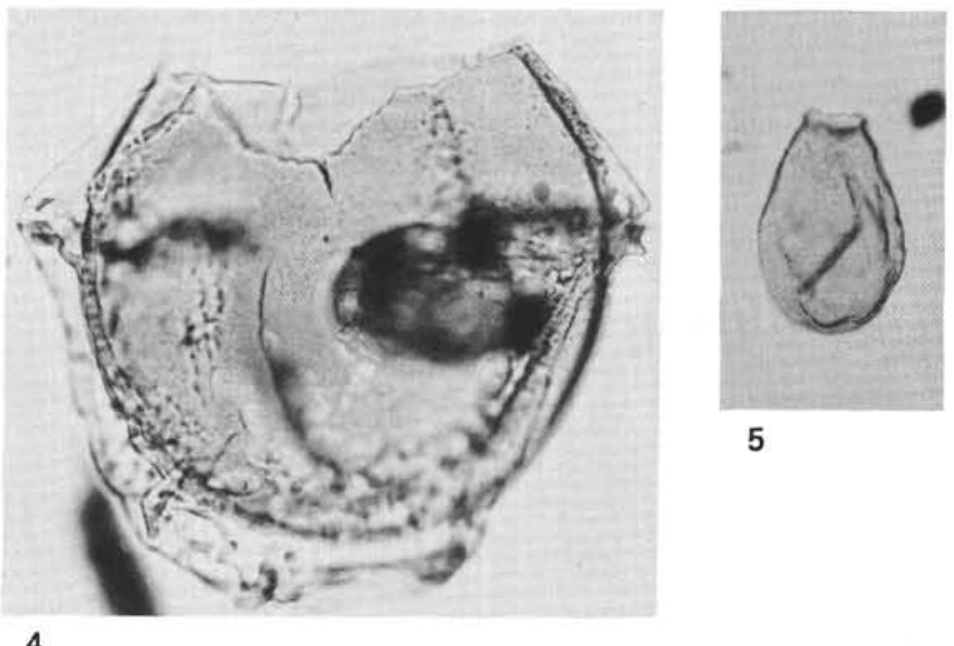

5

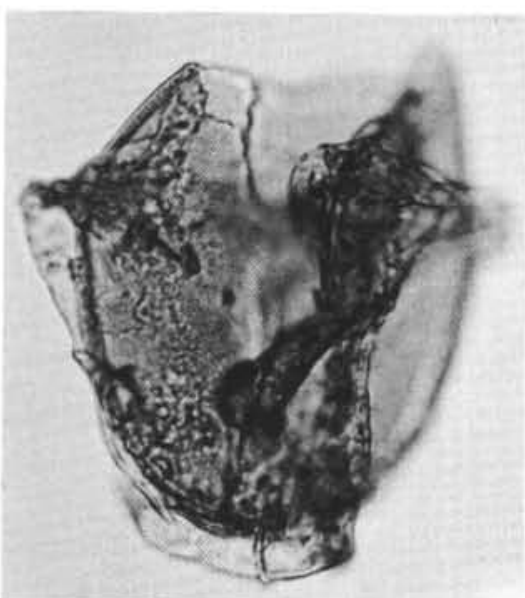

7

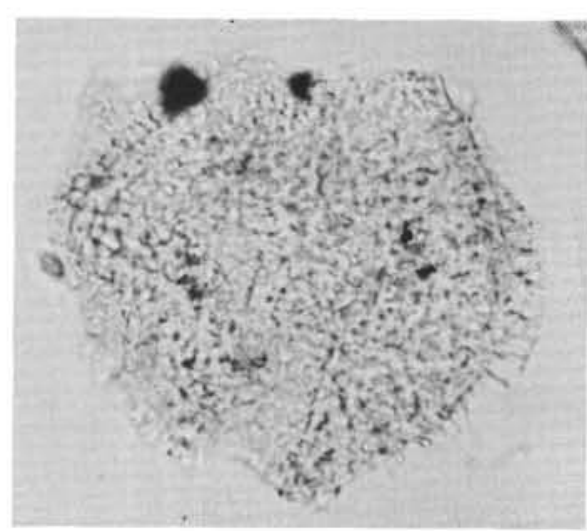

8 


\section{PLATE 18}

All magnifications $\times 500$ unless otherwise stated

Figure 1 Odontochitina operculata (O. Wetzel). Sample 327A-22-1, 126-129 cm. Slide S3103/1, 13.9:102.3.

Figure 2 Odontochitina cribropoda Deflandre and Cookson. Sample 328-12, CC. Slide ST3195/3, 16.2:107.0.

Figure 3 ?Odontochitina costata Alberti. Sample 328B-7-2, $28-31 \mathrm{~cm}$. Slide ST3198/1, 10.4:104.6, operculum.

Figure 4 Odontochitina operculata (O. Wetzel). Sample 327A-22-1, 126-129 cm. Slide S3103/1, 8.7:100.1.

Figure 5 Odontochitina sp. A. Sample 327A-12-2, 99-104 $\mathrm{cm}$. Slide ST3097/3, 14.6:107.8.

Figures 6-8 Odontochitina porifera Cookson.

6. Sample 328-12-1, 69-71 cm. Slide ST3192/2, 12.9:105.3, operculum.

7. Sample $328-11-1,42-44 \mathrm{~cm}$. Slide ST3182/4, 16.2:108.3.

8. Sample $328-11-1,42-44 \mathrm{~cm}$. Slide ST3182/3, 109.6:15.9. 


\section{PLATE 18}
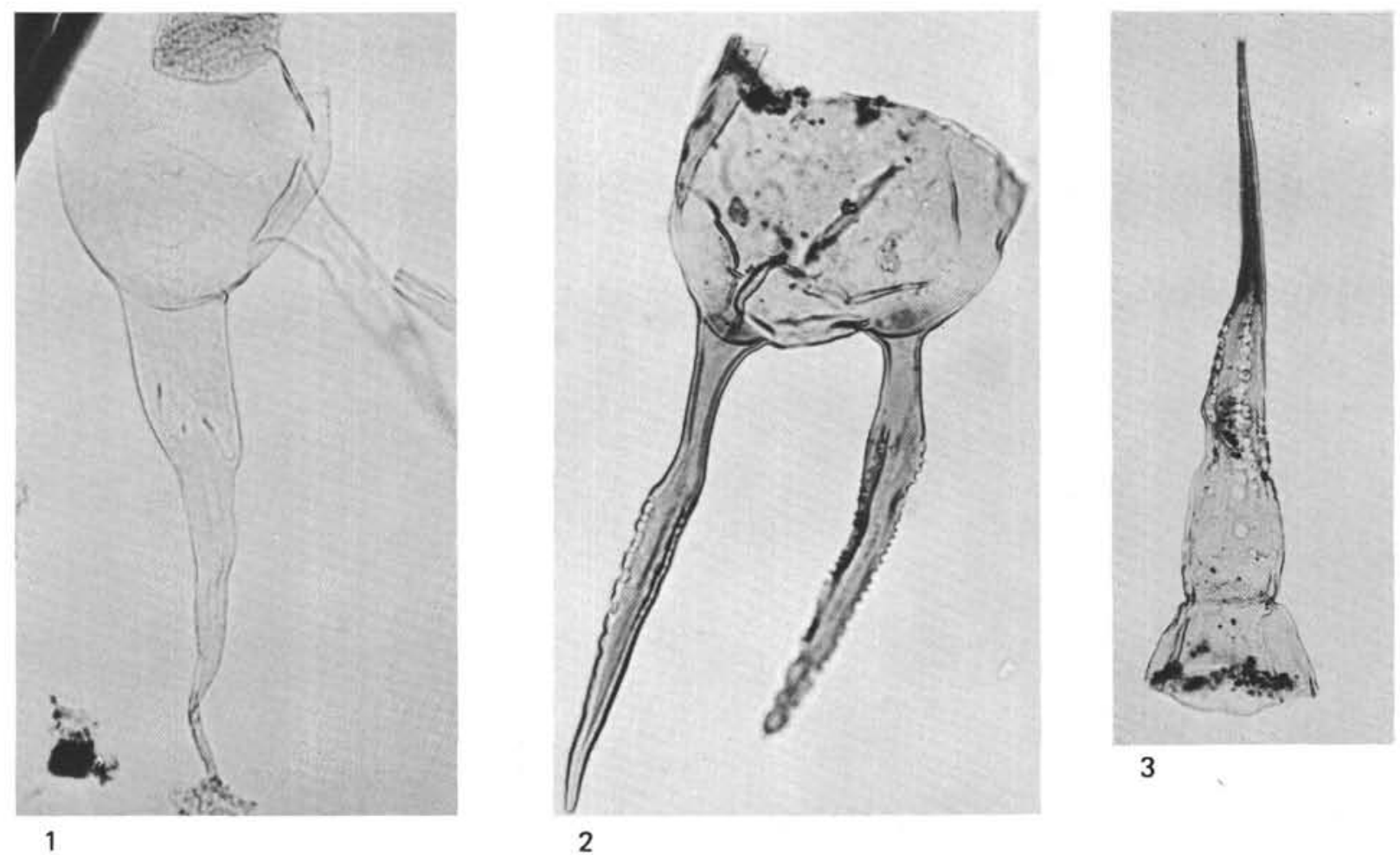

3

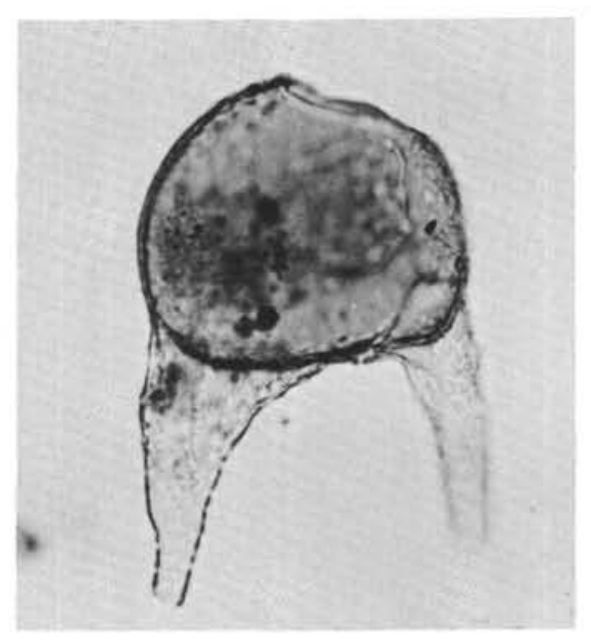

5

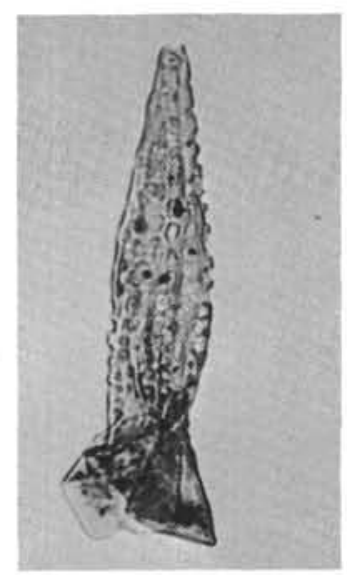

6
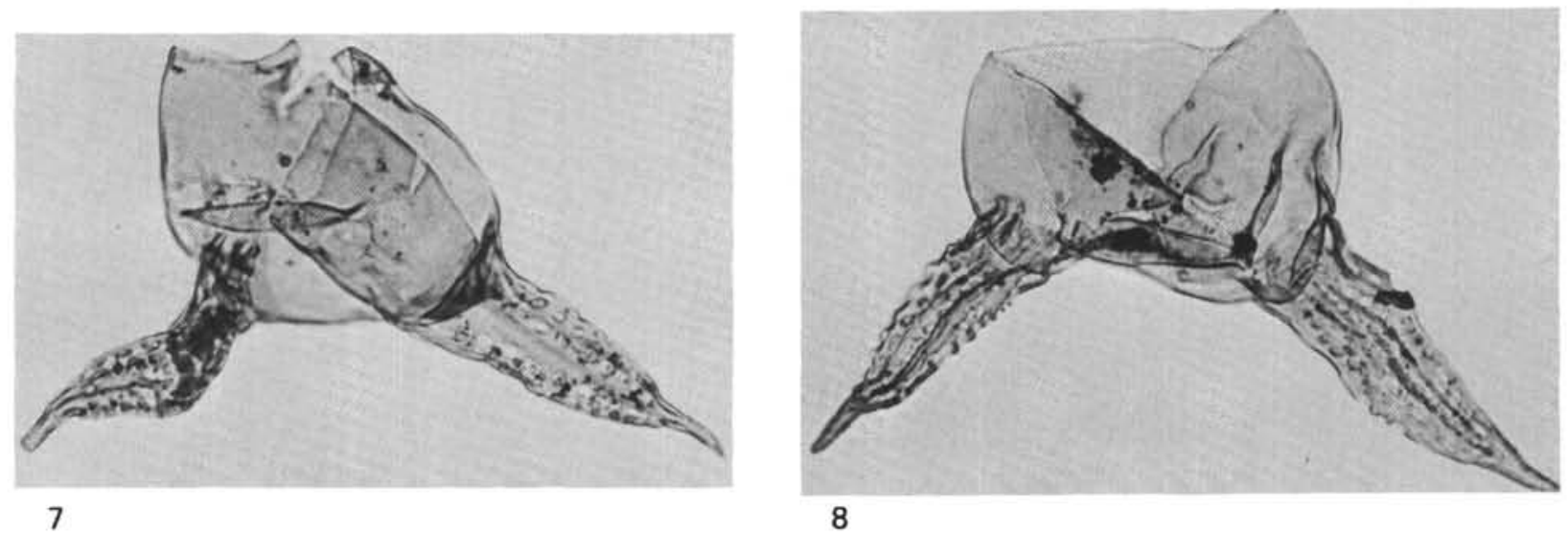


\section{PLATE 19}

All magnifications $\times 500$ unless otherwise stated

Figure 1 Odontochitina sp. A. Sample 327A-12-2, 99-104 $\mathrm{cm}$. Slide ST3097/1, 10.1:112.0.

Figures 2-4 Belodinium sp. aff. B. dysculum.

2. Sample 330-3-2, 103-105 cm. Slide S3157/2, 20.0:104.6.

3, 4. Sample 330-3-2, 103-105 cm. Slide S3157/2, 14.2:117.4.

Figures 5-7 Eisenackia crassitabulata Deflandre and Cookson. Sample 327A-12-3, 116-122 cm. Slide ST3098/1, 9.8:104.1.

Figures 8-11 Eisenackia sp. aff. E. circumtabulata Drugg.

8, 9. Sample 327A-9, CC. Slide ST3093/11, 12.4:108.5.

10, 11. Sample 327A-9, CC. Slide ST3093/6, 8.6:110.0.

Figures 12,13 Membranilarnacia sp. Sample 327A-12-2, 99-104 $\mathrm{cm}$. Slide ST3097/5, 8.6:107.2.

Figures 14, 15 Membranilarnacia sp. Sample 327A-11-1, 65-70 $\mathrm{cm}$. Slide ST3095/1, 11.9:101.2.

Figures 16, 17 Aiora fenestrata (Deflandre and Cookson). Sample 328B-7-6, 4-7 cm. Slide ST3202/3, 10.9:105.4. 
PLATE 19
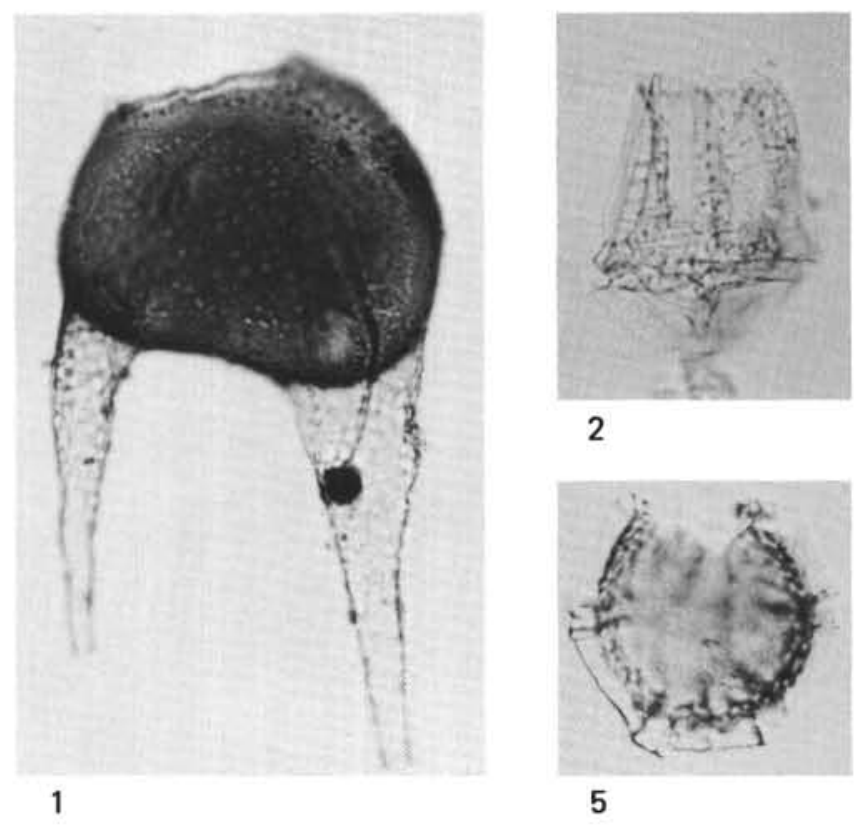

2

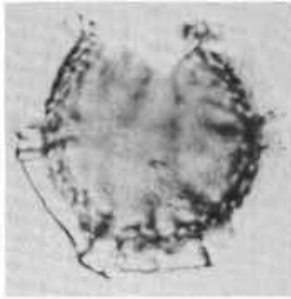

5

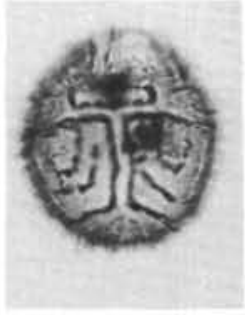

8

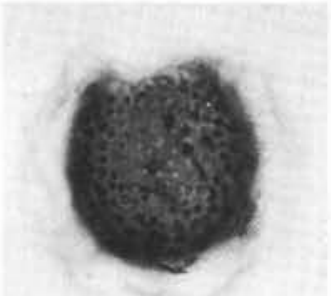

12

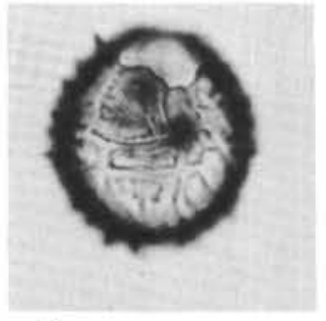

9

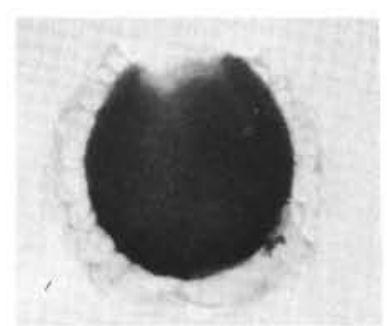

13

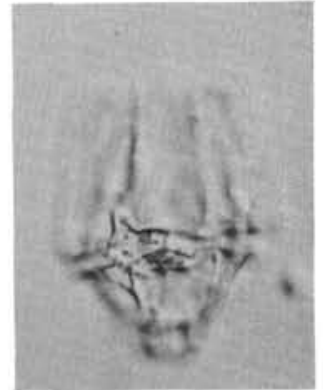

3

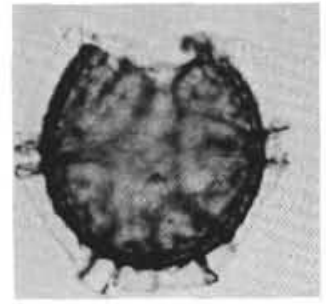

6

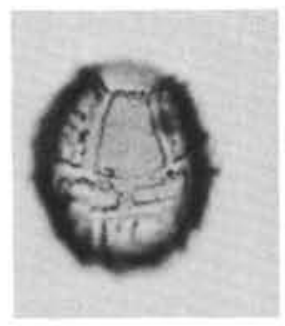

10

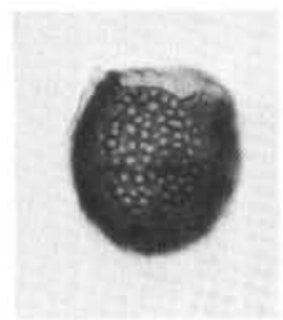

14

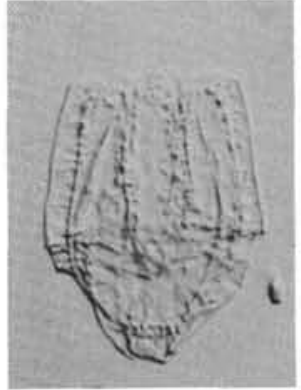

4

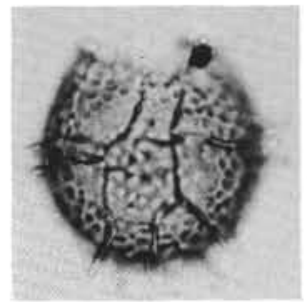

7

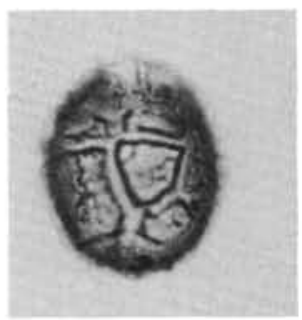

11

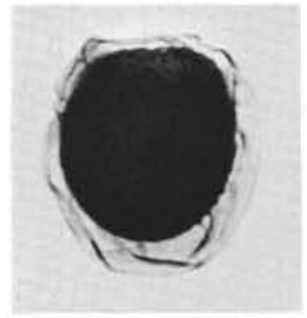

15

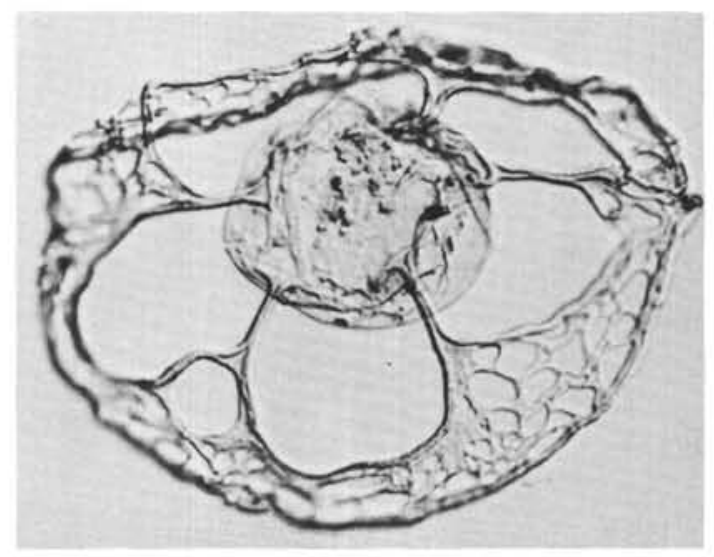

16

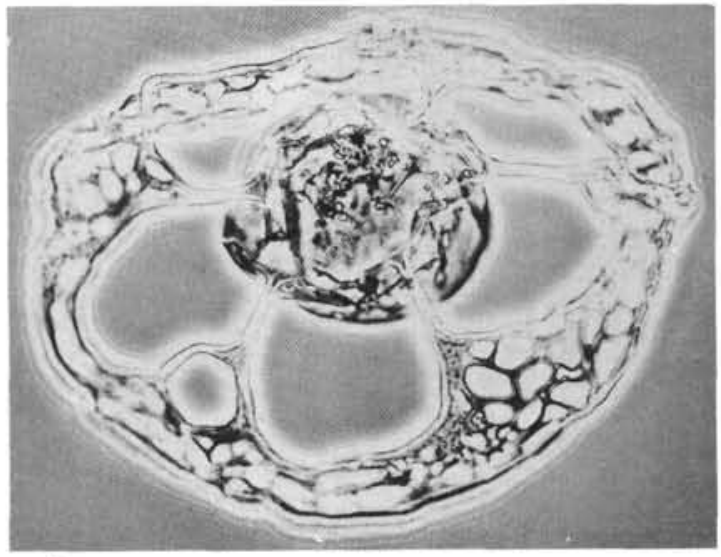

17 


\section{PLATE 20}

All magnifications $\times 500$ unless otherwise stated

Figures 1, 3 Aiora fenestrata (Deflandre and Cookson). Sample 328B-7-3, i04-106 cm. Slide ST3199/3, 11.8:106.5.

Figures 2, 4 Scriniodinium luridum (Deflandre). Sample 330-126, 120-122 cm. Slide S3130/1, 19.9:125.8.

Figure $5 \quad$ ?Pseudoceratium $\mathrm{sp}$. Sample 330-10-1, 129-134 cm. Slide S3141/1, 11.8:133.5.

Figure $6 \quad$ Muderongia simplex Alberti. Sample 330-3-2, 103$105 \mathrm{~cm}$. Slide S3157/2, 17.7:102.3.

Figures 7,8 Scriniodinium luridum (Deflandre).

7. Sample 330-5-1, 143-146 cm. Slide S3161/1, 7.6:114.7.

8. Sample 330-5-1, 143-146 cm. Slide S3161/1, 4.8:100.4.

Figure 9 Dinopterygium cladoides Deflandre. Sample 32812-1, 69-71 cm. Slide ST3192/3, 10.3:107.3. 
PLATE 20

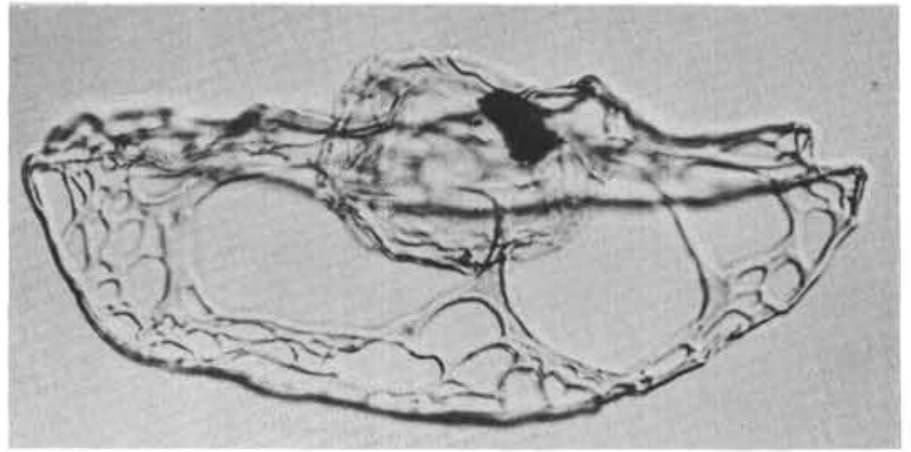

1

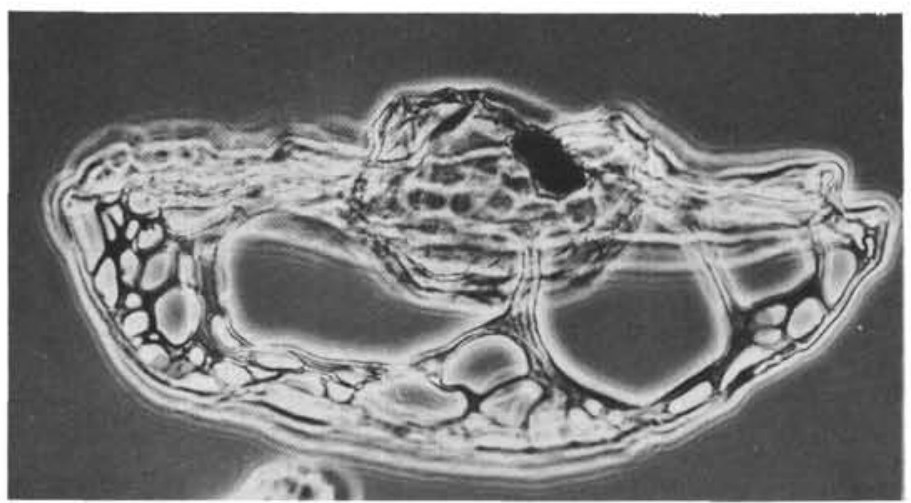

3

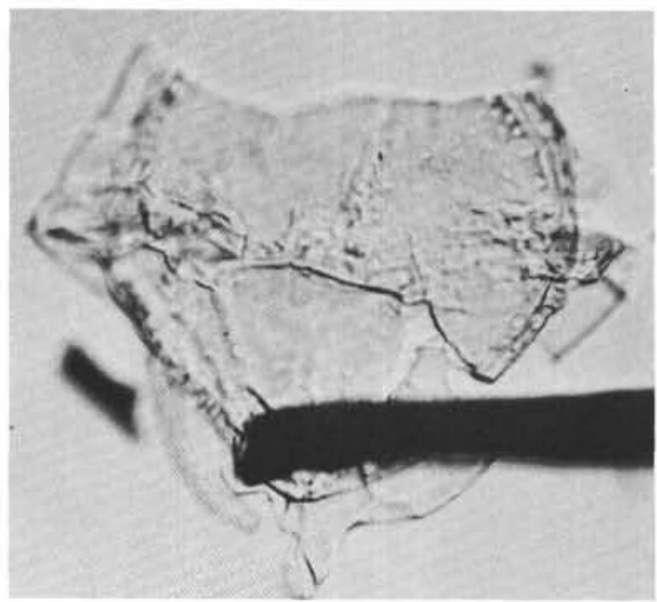

5

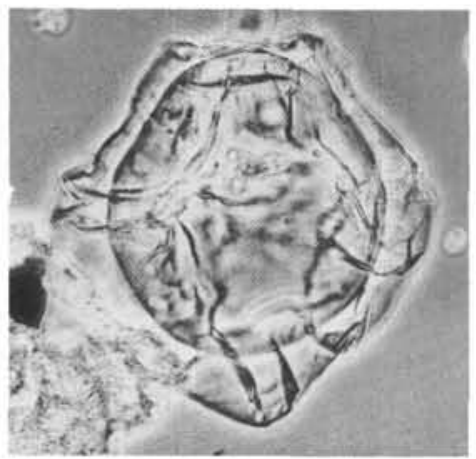

7

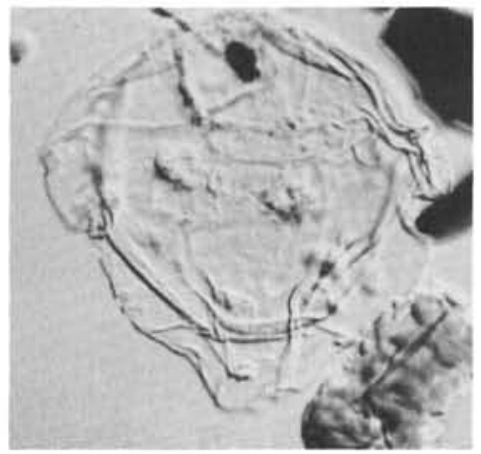

8

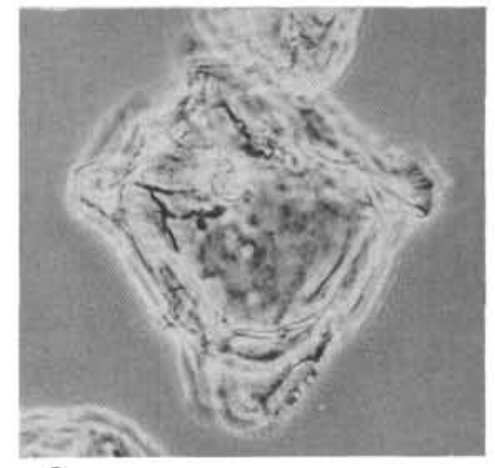

2
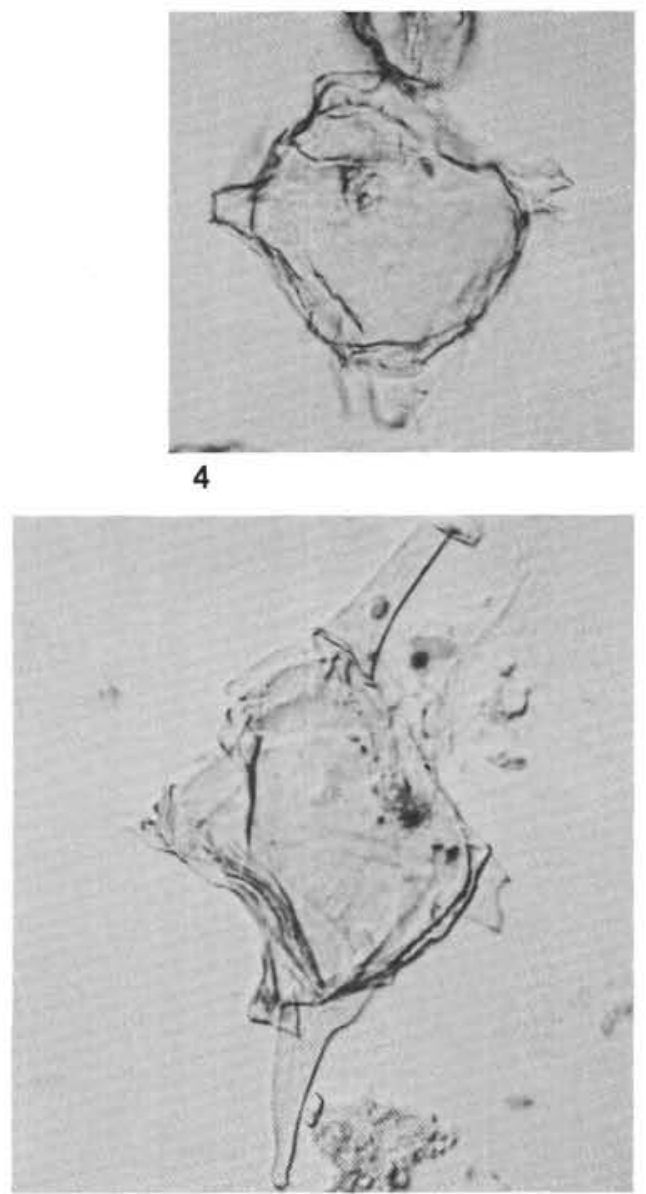

6

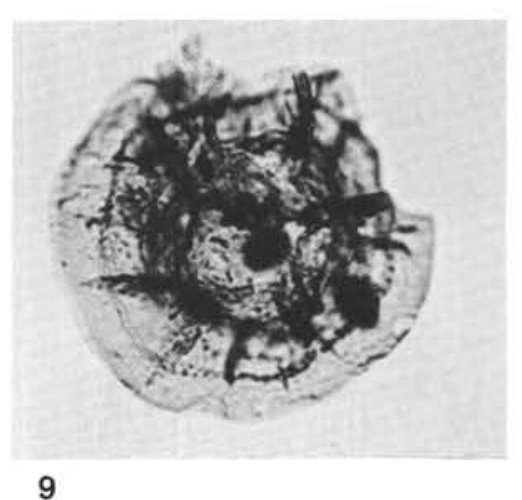




\section{PLATE 21}

All magnifications $\times 500$ unless otherwise stated

Figures 1, 2 Dingodinium cerviculum Cookson and Eisenack. 1. Sample 330-3-2, 103-105 cm. Slide S3157/2, 7.8:99.4.

2. Sample 330-3-2, 103-105 cm. Slide S3157/2, 15.2:123.6.

Figure 3 Dingodinium alberti Sarjeant. Sample 330-4-2, 131$133 \mathrm{~cm}$. Slide S3162/3, 15.5:104.8.

Figure 4 Dingodinium sp. Sample 330-4-2, 131-133 cm. Slide S3162/1, 111.2:13.9.

Figures 5,6 Sp. indet. Sample 330-4-2, 131-133 cm. Slide S3162/3, 15.4:130.0.

Figure 7

Sp. indet. Sample 330-5-1, 143-146 cm. Slide S3161/1, 5.7:133.0.

Figure $8 \quad \mathrm{Sp}$. indet. Sample $330-5-1,143-146 \mathrm{~cm}$. Slide S3161/1, 4.8:106.4.

Figures 9, 10 Sp. indet. Sample 327A-9, CC. Slide ST3093/7, 8.7:108.6.

Figures 11, 12 Hystrichosphaeridium sp. aff. H. pachydermum Cookson and Eisenack. Sample 330-3-2, 103-105 $\mathrm{cm}$. Slide S3157/2, 14.3:107.4.

Figure 13 Sp. indet. Sample 330-14-4, $148-150 \mathrm{~cm}$. Slide S3127/1, 20.2:117.6.

Figure 14 Sp. indet. Sample 327A-22-1, 126-129 cm. Slide S3103/1, 5.9:115.3.

Figures 15, 16 Sp. indet. Sample 328-12, CC. Slide ST3195/6, 12.3:108.5.

Figure 17 Chlamydophorella nyei Cookson and Eisenack. Sample 330-3-2, 103-105 cm. Slide S3157/2, 20.3:106.4.

Figure 18 Sp. indet. Sample 330-12-6, 120-122 cm. Slide S3130/1, 5.4:100.0.

Figure 19 Sp. indet. Sample 330-12-6, 120-122 cm. Slide S3130/1, 7.1:107.9.

Figure 20 Sp. indet. Sample 327A-22-1, 126-129 cm. Slide S3103/1, 15.4:116.9.

Figure $21 \quad$ Sp. indet. Sample 327A-22-1, 126-129 cm. Slide S3103/1, 6.0:124.3.

Figure 22 Sp. indet. Sample 330-4-2, 131-133 cm. Slide S3162/3, 13.2:108.9. 
PLATE 21

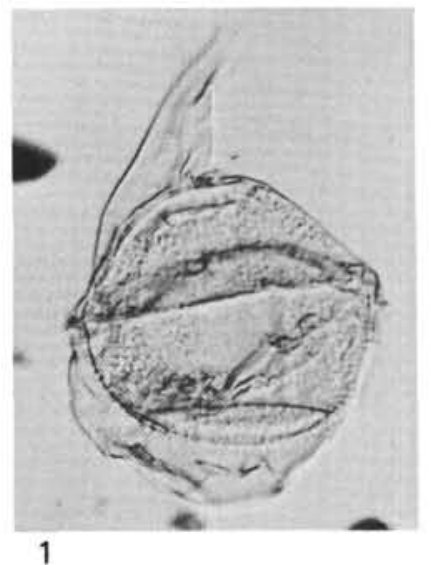

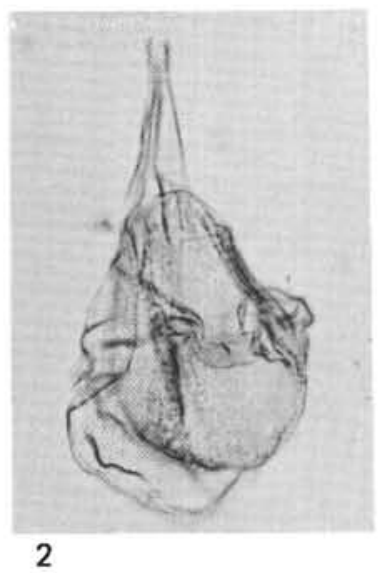

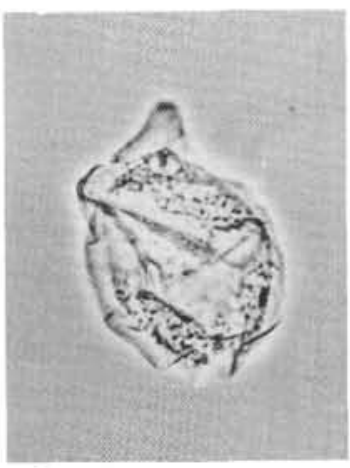

3

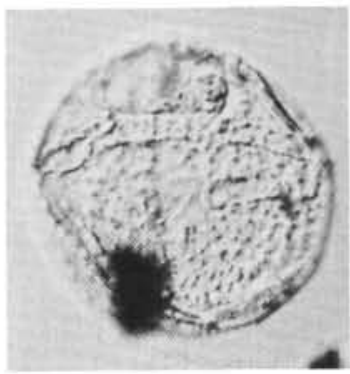

7

6

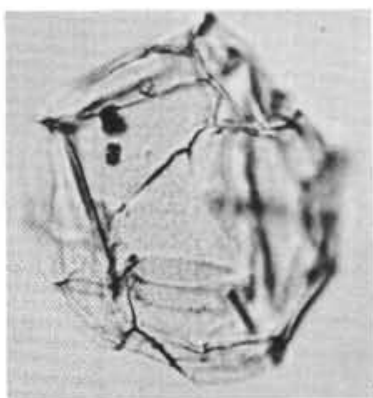

10

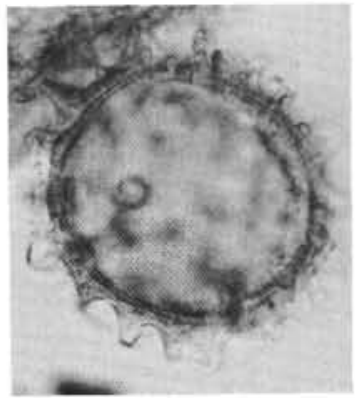

11
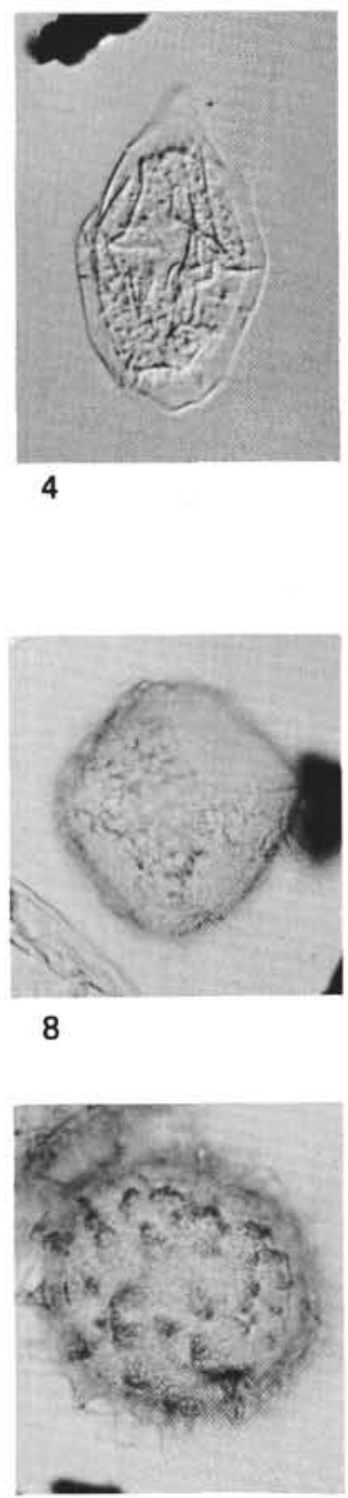

12

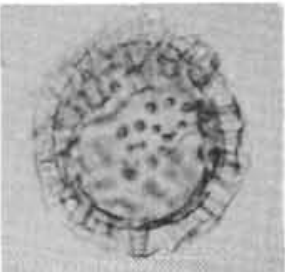

17

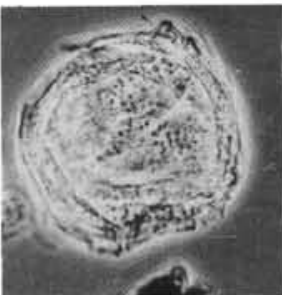

22 


\section{PLATE 22}

All magnifications $\times 500$ unless otherwise stated

Figure 1 ?Canningia sp. Sample $328-11-3,66-68 \mathrm{~cm}$. Slide ST3183/4, 15.4:107.8.

Figure 2 Pterospermella $\mathrm{sp}$. Sample $330-4-1,86-88 \mathrm{~cm}$. Slide S3163/1, 5.4:109.3.

Figure 3 Palaeostomocystis sp. Sample 327A-8, CC. Slide ST3092/2, 13.1:108.1.

Figure $4 \quad$ Pterospermella australiensis (Deflandre and Cookson). Sample 330-3-2, 103-105 cm. Slide S3157/2, 7.4:129.6.

Figure 5 Palaeostomocystis sp. Sample 327A-8, CC. Slide ST3092/3, 12.5:105.4.

Figures 6,7 Kalyptea $\mathrm{sp}$.

6. Sample 330-3-2, 103-105 cm. Slide S3157/2, 8.9:102.3.

7. Sample $330-3-2,103-105 \mathrm{~cm}$. Slide S3157/2, 16.8:113.7.

Figure 8 Pterospermella sp. Sample 327A-22-2, 7-10 cm. Slide ST3105/13, 10.5:117.7.

Figure 9 Sp. indet. Sample 330-3-2, 103-105 cm. Slide S3157/2, 11.8:108.1. 


\section{PLATE 22}

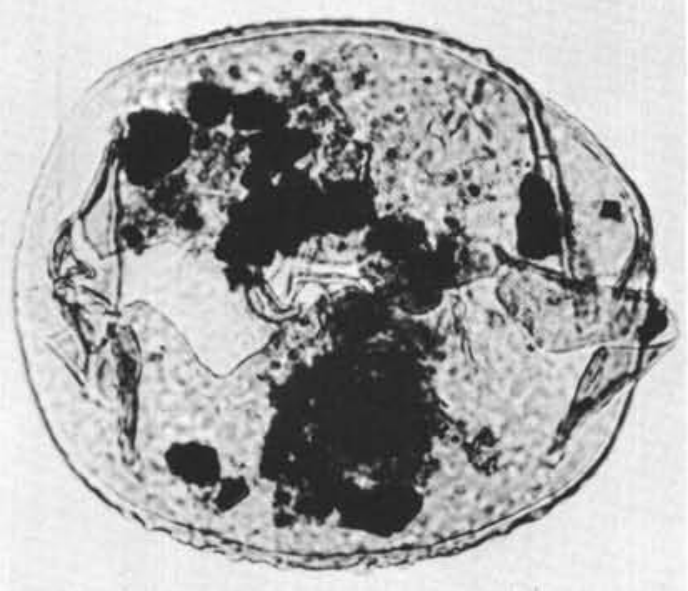

1

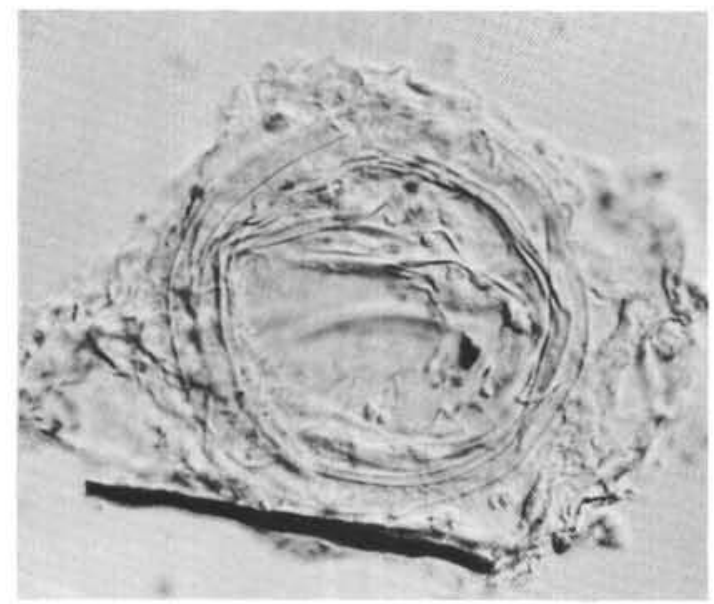

6

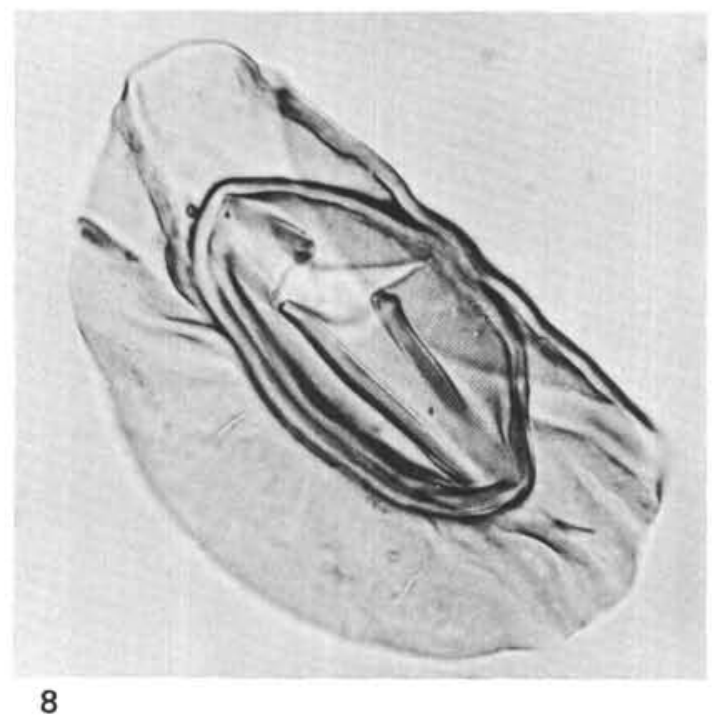

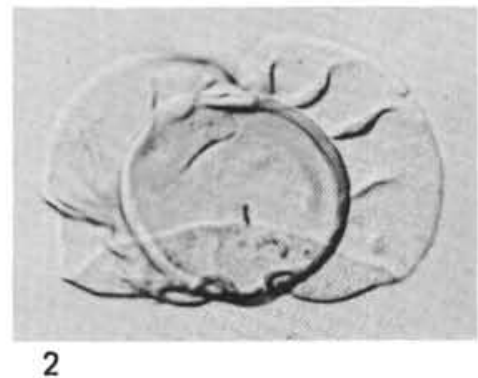
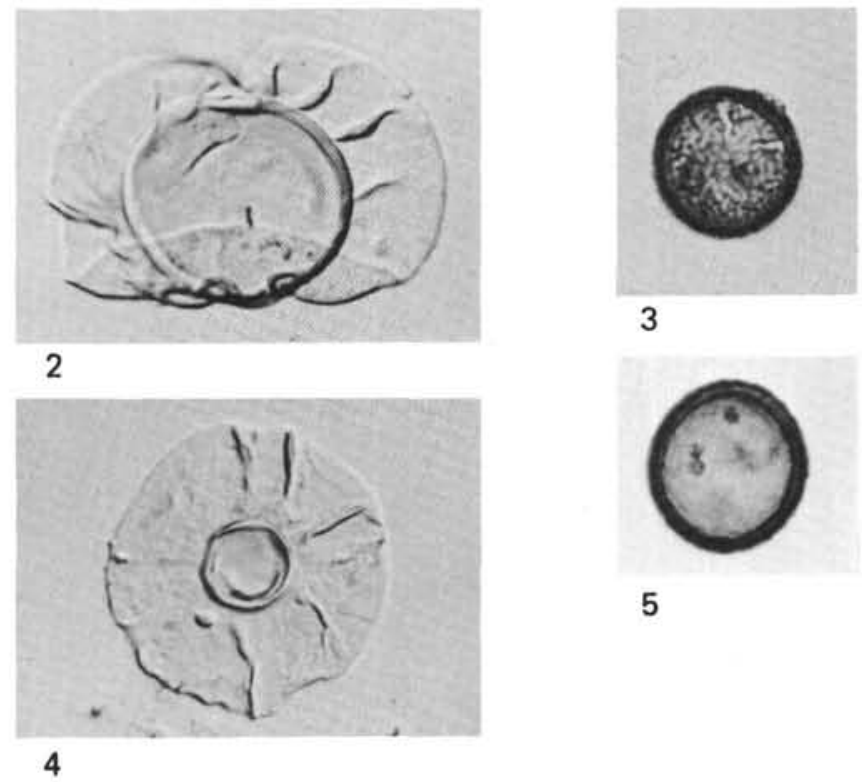

5

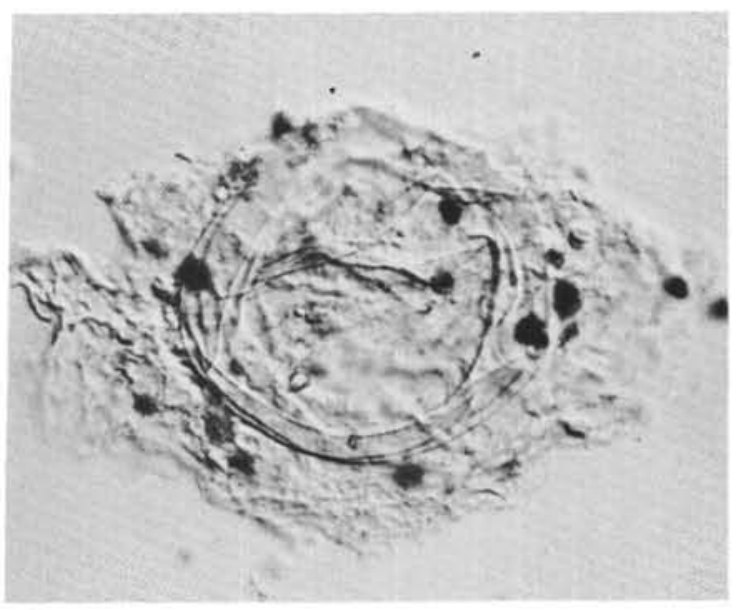

7

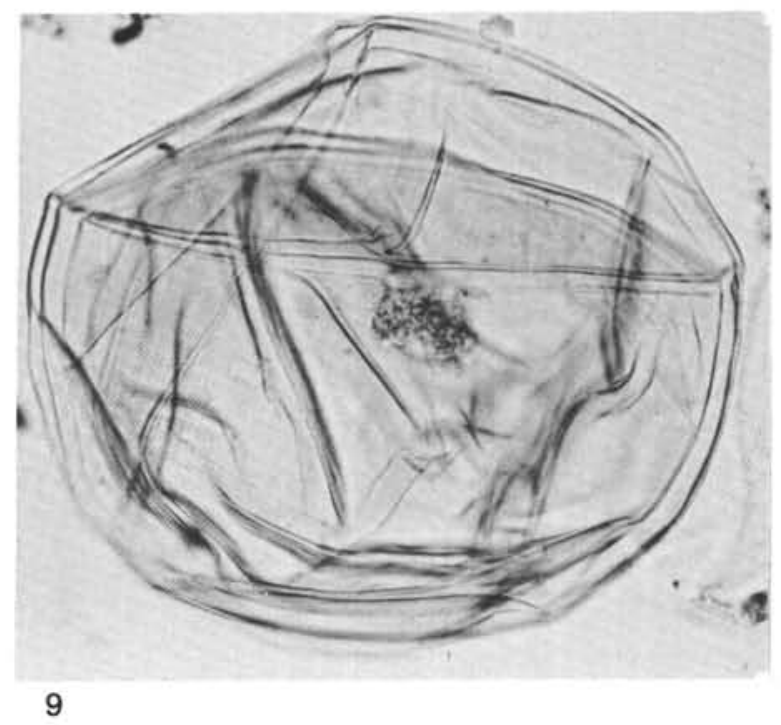


PLATE 23

All magnifications $\times 500$ unless otherwise stated

Figures 1,2 ?Araucariacites $\mathrm{sp}$.

1. Sample 330-4-2, 131-133 cm. Slide S3162/1, 12.0:127.0.

2. Sample 330-4-2, 131-133 cm. Slide S3162/3, 6.7:96.9.

Figures 3-5 ? Inaperturopollenites $\mathrm{sp}$.

3. Sample 327 A-22-2, 7-10 cm. Slide ST3105/14, 14.2:110.6.

4. Sample 327 A-22-2, 7-10 cm. Slide ST3105/24, 11.2:105.5.

5. Sample 327 A-22-2, 7-10 cm. Slide ST3105/21, 12.6:109.1.

Figures 6, 7, 10 Tasmanites suevicus (Eisenack).

6. Sample $330-8-4,98-100 \mathrm{~cm}$. Slide S3143/1, 5.0:125.1.

7, 10. Sample 330-10-1, 129-134 cm. Slide S3141/1, 14.7:108.2.

Figure $8 \quad$ Lecaniella $\mathrm{sp}$. Sample $330-8-4,98-100 \mathrm{~cm}$. Slide S3143/1, 11.9:107.6.

Figure 9 Tasmanites sp. Sample 330-3-2, 103-105 cm. Slide S3157/2, 15.6:105.1.

Figure 11 Lecaniella foveolata Filatoff. Sample 330-4-2, 131$133 \mathrm{~cm}$. Slide S3162/3, 4.6:104.0.

Figures 12, 14 Tasmanites suevicus (Eisenack). Sample 330-10-1, 129-134 cm. Slide S3141/1, 8.7:108.9.

Figure 13 Diplotesta sp. aff. D. sp. Sample 330-4-2, 131-133 $\mathrm{cm}$. Slide S3162/3, 10.9:99.1. 
PLATE 23

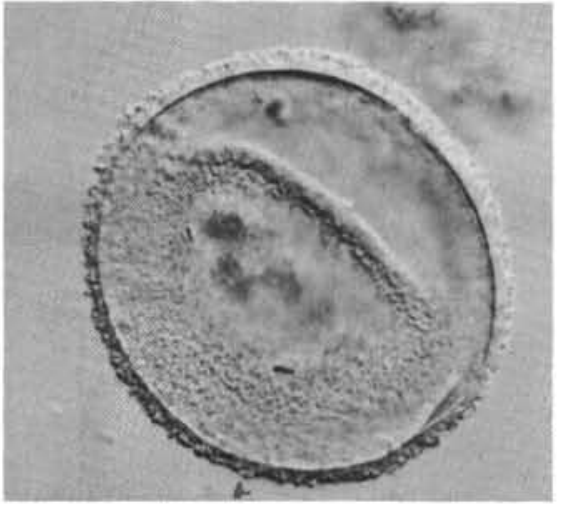

1

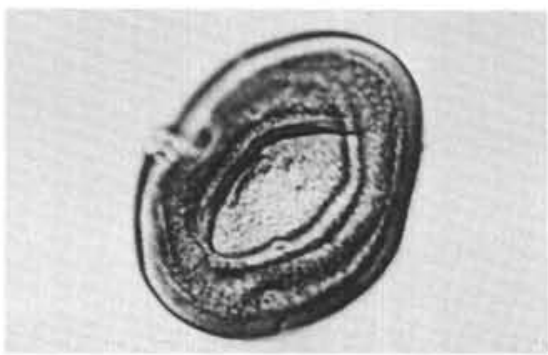

4
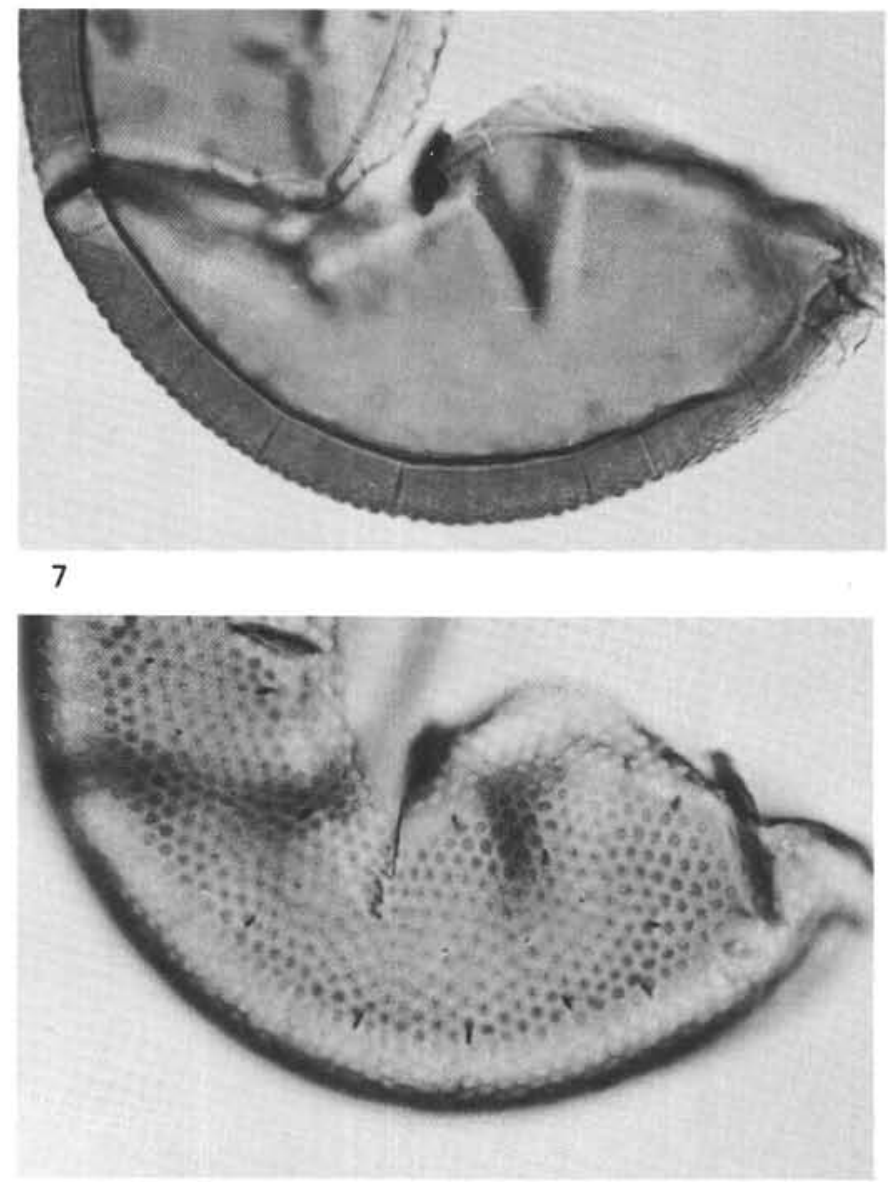

2

5
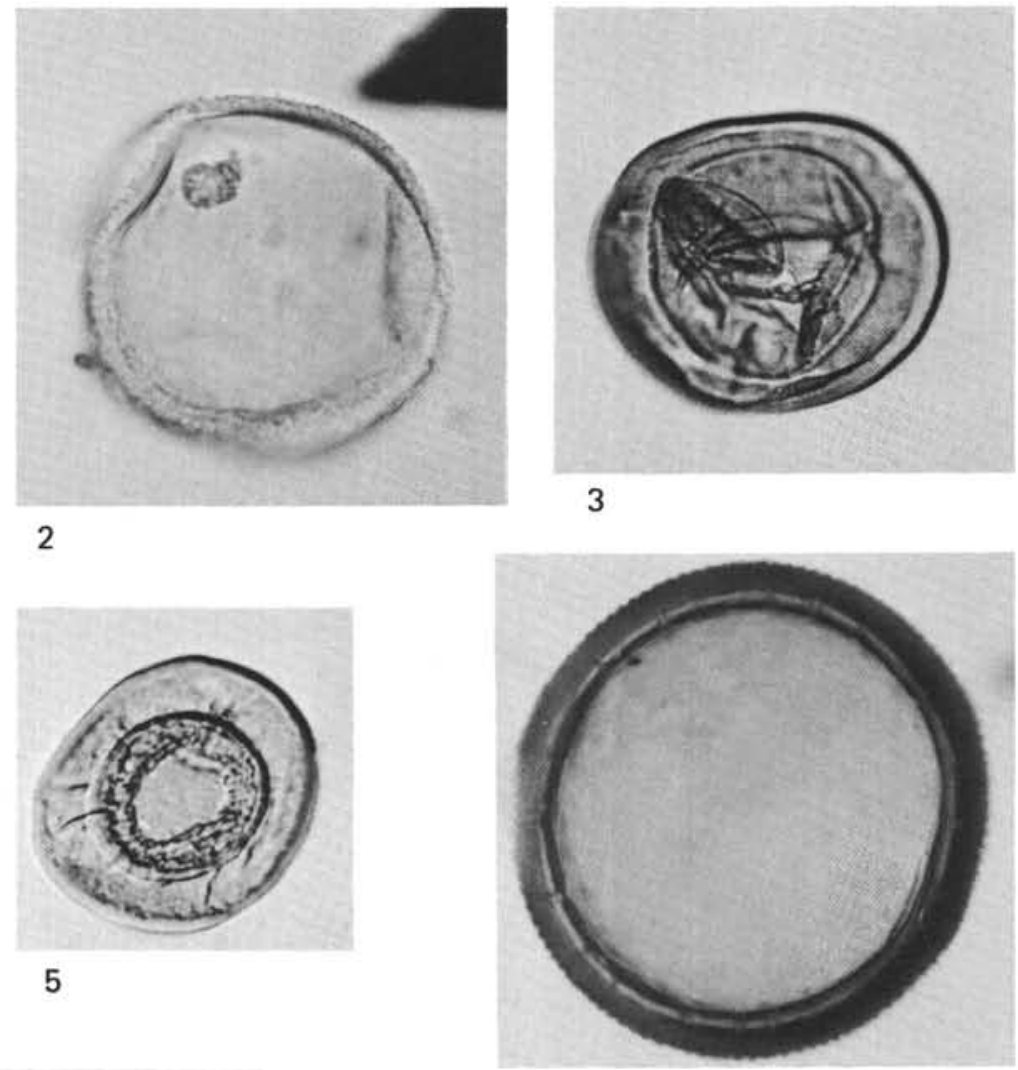

6
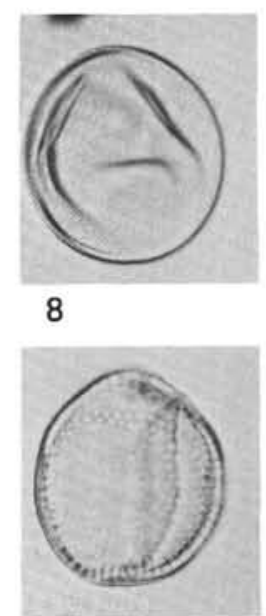

11

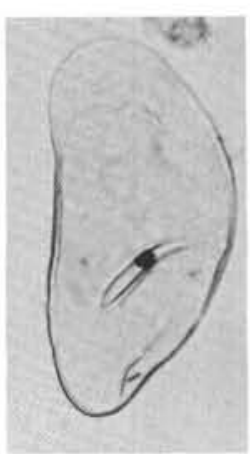

13
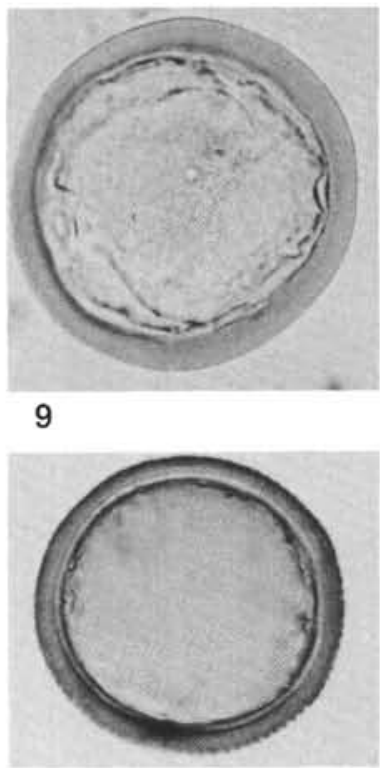

12

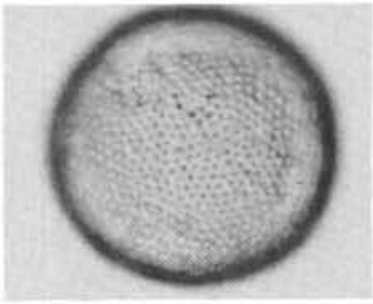

14 


\section{PLATE 24}

All magnifications $\times 500$ unless otherwise stated

Figure 1 Tasmanites $\mathrm{sp}$. Sample 330-11-4, $42-46 \mathrm{~cm}$. Slide S3136/1, 7.9:116.9.

Figure 2 Tasmanites $\mathrm{sp}$. Sample $330-13-2,31-33 \mathrm{~cm}$. Slide S3129/1, 11.7:106.1.

Figure 3 Cometodinium sp. Sample 327A-22-1, 126-129 cm. Slide S3103\&1, 6.0:100.5.

Figure 4 Veryhachium sp. Sample 327A-22-1, 126-129 cm. Slide S3103/1, 4.9:99.6.

Figures 5,6 Leiosphaeridia $\mathrm{sp}$.

5. Sample 330-12-6, 120-122 cm. Slide S3130/1, 4.8:114.6.

6. Sample 330-3-2, 103-105 cm. Slide S3157/2, 4.1:106.1.

Figures 7, 8 Micrhystridium sp.

7. Sample 330-12-6, 120-122 cm. Slide S3130/1, 5.0:99.7.

8. Sample 330-11-5, 126-128 cm. Slide S3135/1, 3.9:105.7.

Figures 9-12 Cymatosphaera spp.

9. Sample 330-7-2, 55-59 cm. Slide S3159/1, 5.1:123.2.

10. Sample 330-7-2, $55-59 \mathrm{~cm}$. Slide S3159/1, 7.3:126.6.

11, 12. Sample 330-3-2, 103-105 cm. Slide S3157/2, 6.9:122.1.

Figures 13-16 Pterosphaeridia $\mathrm{sp}$. aff. P. pachytheca (Eisenack). 13, 14. Sample 330-3-2, 103-105 cm. Slide S3157/2, 18.4:119.8.

15, 16. Sample 330-3-2, 103-105 cm. Slide $\mathrm{S} 3157 / 2,12.5: 120.0$.

Figures 17, 18 Gen. et ap. indet. Sample 330-3-2, 103-105 cm. Slide S3157/2, 15.2:113.7.

Figure 19 Sp. indet. Sample 327A-9, CC. Slide ST3093/10, 13.2:108.2. 
PLATE 24

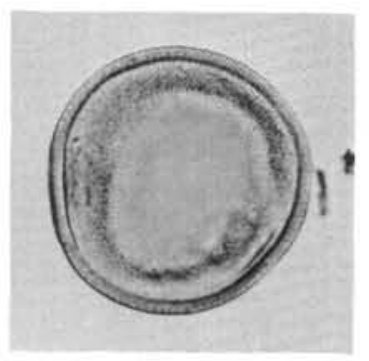

1

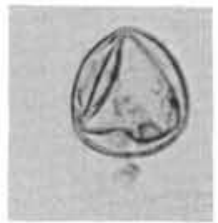

5

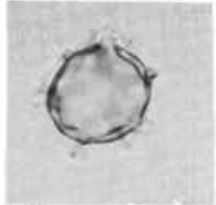

11

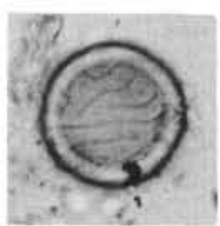

17

6

12

18

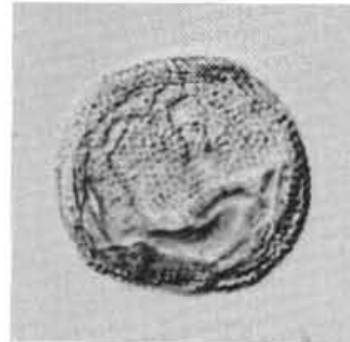

2
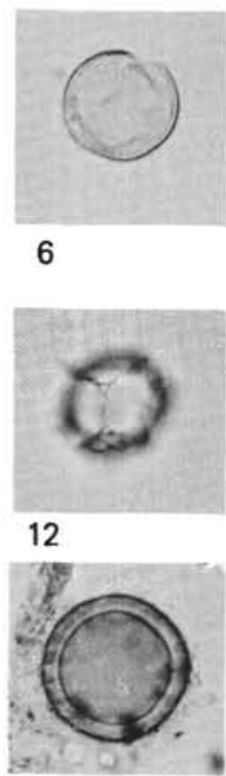

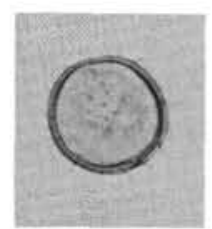

7

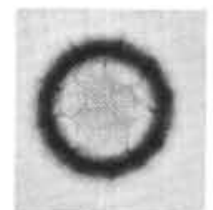

13

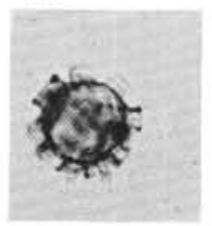

19

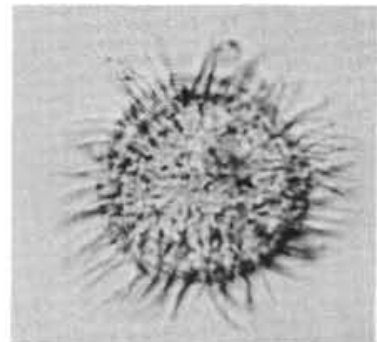

3

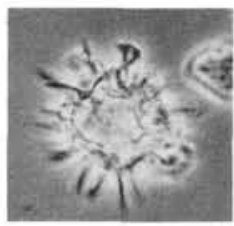

8

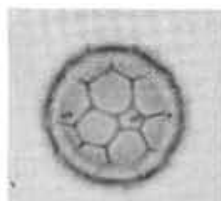

14

15

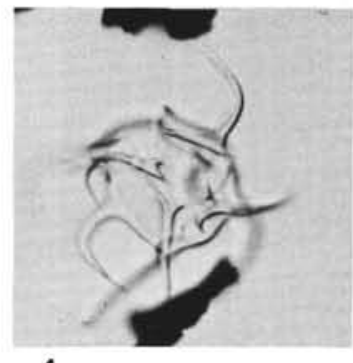

4
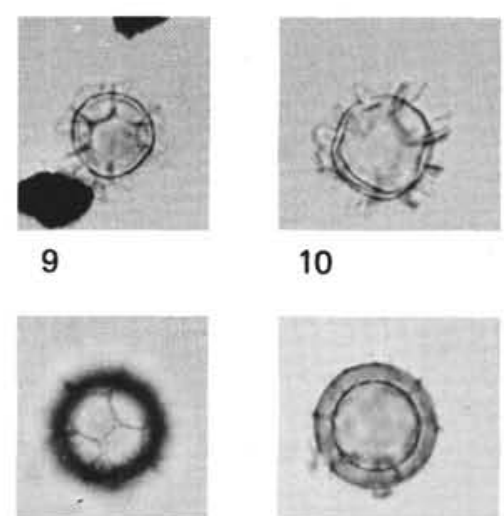

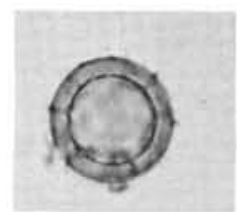

16 